Aus der Abteilung Hals-Nasen-Ohrenheilkunde

(Prof. Dr. med. C. Matthias)

im Zentrum Augenheilkunde und Hals-Nasen-Ohrenheilkunde

der Medizinischen Fakultät der Universität Göttingen

\title{
Langzeitbeobachtungen zur Injektionsdynamik der \\ Botulinum-Toxin-Therapie bei Patienten mit verschiedenen fazialen Dyskinesien
}

\author{
INAUGURAL -DISSERTATION \\ zur Erlangung des Doktorgrades \\ der Medizinischen Fakultät \\ der Georg-August-Universität zu Göttingen
}

vorgelegt von

Anna Maria Niemczewska-Dreher

aus

Kwidzyn (Polen)

Göttingen 2013 
D e k a n:

I. Berichterstatter:

II. Berichterstatter/in:

III. Berichterstatter/in:

Tag der mündlichen Prüfung: 25.11.2013
Prof. Dr. med. Heyo K. Kroemer

Prof. Dr. med. R. Laskawi

Prof. Dr. med. Liebetanz

Prof. Dr. med. dent. Mausberg 


\section{Inhaltsverzeichnis}

\section{Abbildungsverzeichnis}

\section{Tabellenverzeichnis}

\section{Abkürzungsverzeichnis}

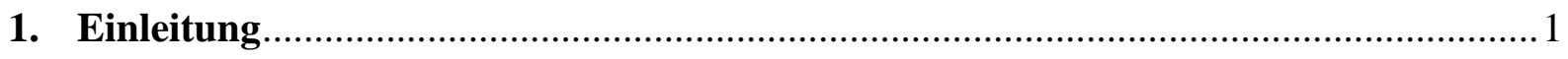

1.1. Anatomie und Innervation der Gesichtsmuskulatur .................................................. 1

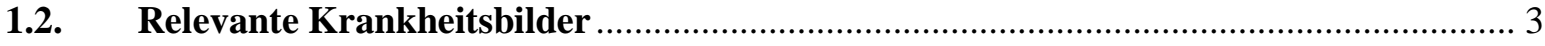

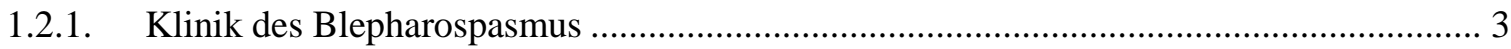

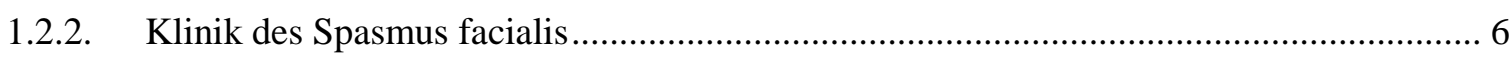

1.2.3. Klinik der Synkinesien nach Fazialisdefektheilung ....................................................... 8

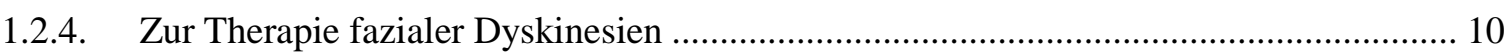

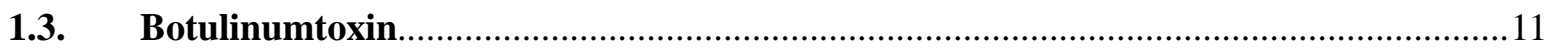

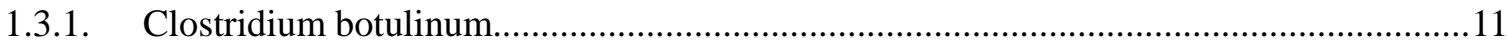

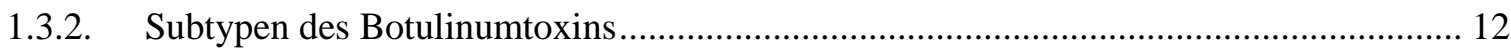

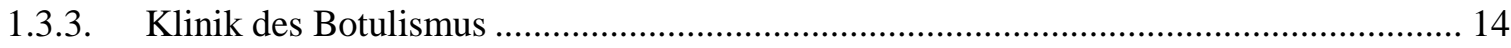

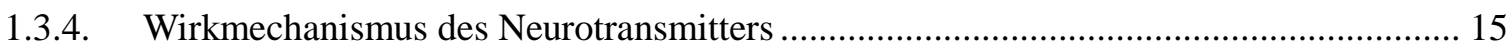

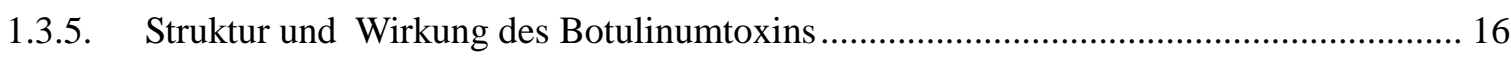

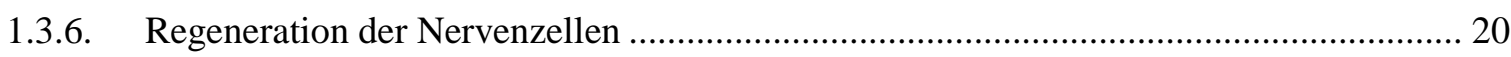

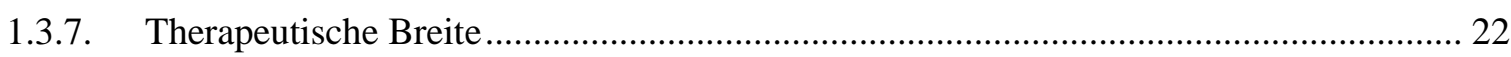

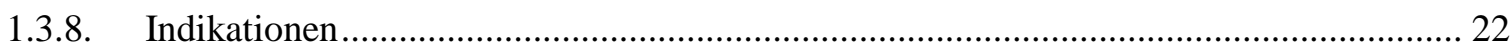

1.3.9. Immunität und unerwünschte Wirkungen..................................................................... 23

1.4. Behandlung mit der Substanz Botulinumtoxin Typ A (Botox $\left.{ }^{\circledR}\right)$.................................. 24

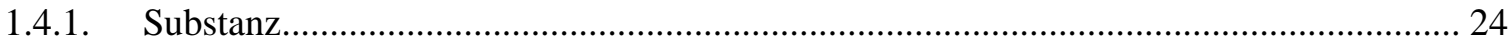

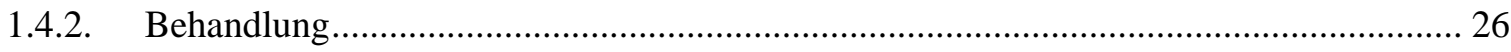

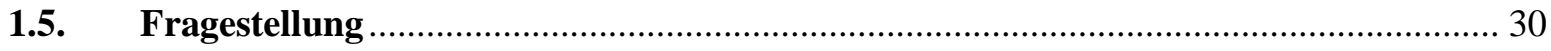

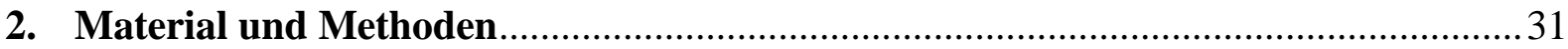

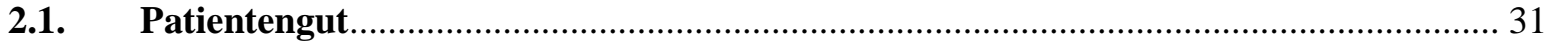

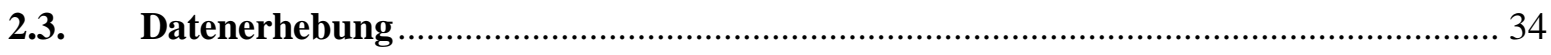

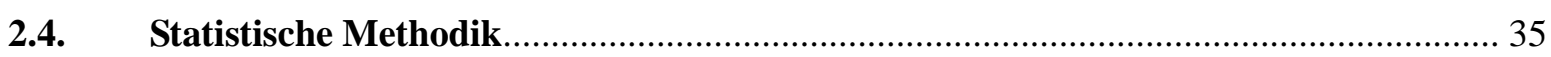

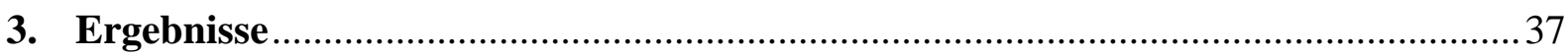

3.1. Botulinumtoxin-Therapie bei fazialen Hyperkinesien................................................. 37

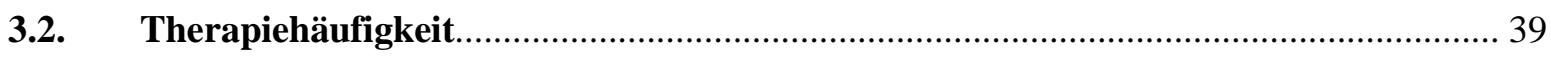

3.2.1. Therapiehäufigkeit bei Blepharospasmus, Spasmus facialis und Synkinesien................ 39

3.3. Entwicklung der durchschnittlichen Gesamtdosis über die Behandlungsjahre .......... 40 
3.4. Entwicklung der Anzahl der Injektionspunkte in den Behandlungsjahren ................. 53

3.5. Dosis-Dynamik der Injektionspunkte bei den fazialen Dyskinesien ............................. 64

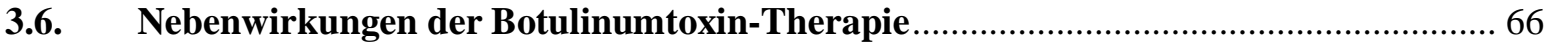

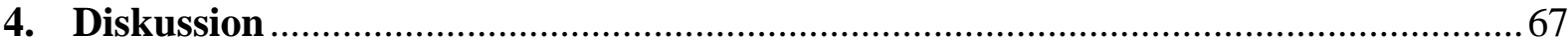

4.1. Wirkung von Botulinumtoxin A bei fazialen Dyskinesien.............................................. 67

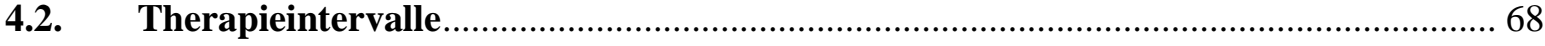

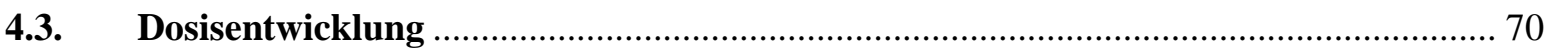

4.4. Dosiserhöhung und deren mögliche Ursachen …......................................................... 74

4.5. Anzahl der Injektionspunkte und die Dosisentwicklung am Injektionsort ................... 77

4.6. Nebenwirkungen der Botulinumtoxin-Therapie ............................................................ 78

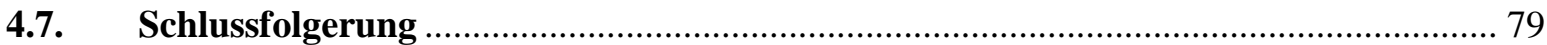

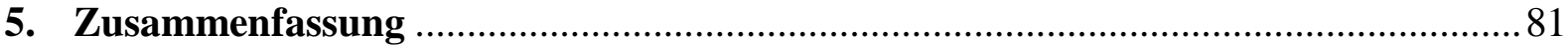

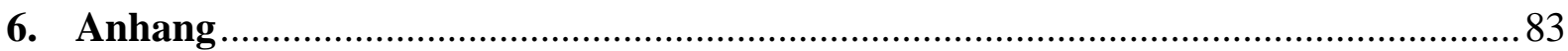

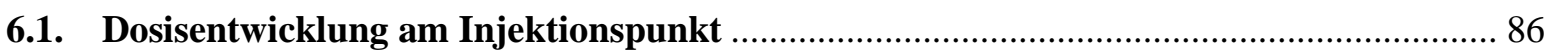

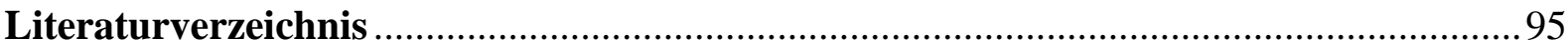




\section{Abbildungsverzeichnis}

Abbildung 1: Anatomie der mimischen Muskulatur (aus Glaser 2009, Seite 134)............. 2

Abbildung 2: $\quad$ Beispiel einer Patientin mit einem Blepharospasmus. ...................................... 3

Abbildung 3: Beispiel eines Patienten mit einem Spasmus facialis.................................... 7

Abbildung 4: Beispiel einer Patientin mit Synkinesien.................................................... 9

Abbildung 5: Entwicklung der Botulinumtoxin-Präparate (aus Dressler 2008, Seite

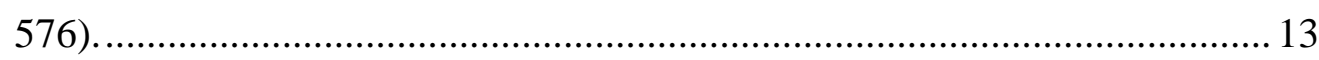

Abbildung 6: Wirkmechanismus des natürlichen Transmitters (aus Arnon et al. 2001,

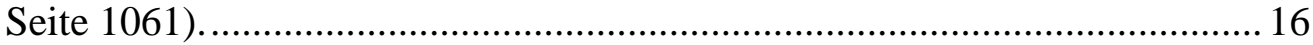

Abbildung 7: Molekülstruktur des Botulinumtoxin A (aus Brunger und Rummel 2009, Seite 551).

Abbildung 8: Modell für Bindung, Vesikelinternalisation und Translokation der leichten

Abbildung 9: Wirkmechanismus von Botulinumtoxin A (aus Frevert 2009, Seite 12)........ 19

Abbildung 10: Regeneration der neuromuskulären Einheit (aus Foran et al. 2003, Seite 294).

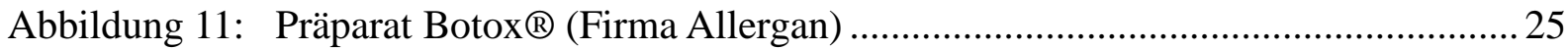

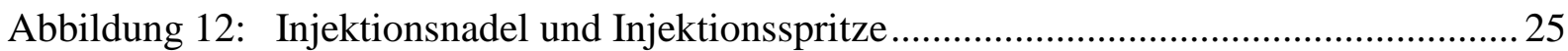

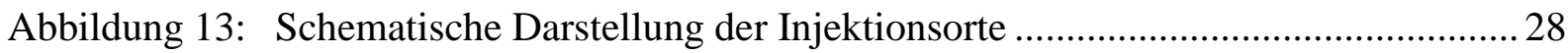

Abbildung 14: Patientenanzahl und Geschlechtsverteilung in den Behandlungsgruppen. .... 31

Abbildung 15: Diagnose der Patienten mit Spasmus facialis. (KHBW=

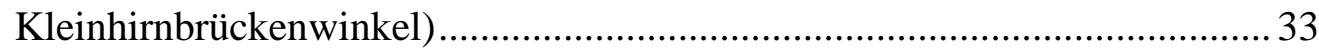

Abbildung 16: Diagnosen der Patienten mit Synkinesien nach Fazialisdefektheilung. ......... 34

Abbildung 17: Verteilung des Alters der Patienten in den Erkrankungsgruppen: .................. 38

Abbildung 18: Mittlere Botulinumtoxin-Dosis mit Standardabweichung (E) bei .................. 41

Abbildung 19: Mittlere Dosis mit Standardabweichung (E) in der....................................... 42

Abbildung 20: Mittlere Dosis mit Standardabweichung (E) in der Spasmus-

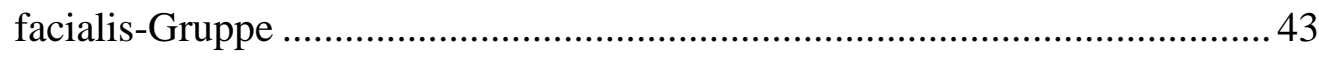

Abbildung 21: Mittlere Dosis und Standardabweichung (E) in der Synkinesien-Gruppe ..... 44

Abbildung 22: Mittlere Dosis (E) mit Konfidenzintervall zum ersten und zehnten Injektionszeitpunkt bei den Erkrankungsgruppen ..................................... 45 
Abbildung 23: Mittlere Dosis (E) mit Konfidenzintervall zum ersten und zwanzigsten Injektionszeitpunkt bei den Erkrankungsgruppen

Abbildung 24: Mittlere Dosis (E) mit Konfidenzintervall zum ersten Injektionszeitpunkt und zum dreißigsten Injektionszeitpunkt bei den Erkrankungsgruppen

Abbildung 25: Mittlere Dosis (E) mit Konfidenzintervall zum ersten I und vierzigsten Injektionszeitpunkt bei den Erkrankungsgruppen

Abbildung 26: Mittlere Injektionspunktanzahl mit Standardabweichung pro Behandlung bei Blepharospasmus ( $\mathrm{n}=30)$, Spasmus facialis $(\mathrm{n}=31)$ und Synkinesien $(\mathrm{n}=19)$.

Abbildung 27: Mittlere Injektionspunktanzahl mit Konfidenzintervall zum ersten und zum zehnten Injektionszeitpunkt bei den Erkrankungsgruppen

Abbildung 28: Mittlere Injektionspunktanzahl mit Konfidenzintervall zum ersten und zwanzigsten Injektionszeitpunkt bei den Erkrankungsgruppen 59

Abbildung 29: Mittlere Injektionspunktanzahl mit Konfidenzintervall zum ersten und dreißigsten Injektionszeitpunkt bei den Erkrankungsgruppen

Abbildung 30: Mittlere Injektionspunktanzahl mit Konfidenzintervall zum ersten und vierzigsten Injektionszeitpunkt bei den Erkrankungsgruppen

Abbildung 31: Entwicklung der durchschnittlichen Dosis pro Injektionspunkt mit Standardabweichung über die Zeit. 65

Abbildung 32: Dokumentationsbogen-Behandlung fazialer Dyskinesien 83

Abbildung 33: Einverständniserklärung Botulinumtoxin-Therapie (Seite 1) 84

Abbildung 34: Einverständniserklärung Botulinumtoxin-Therapie (Seite 2) 85

Abbildung 35: Mittlere Dosis mit Standardabweichung pro Injektionspunkt bei Patienten mit Blepharospasmus.

Abbildung 36: Mittlere Dosis mit Standardabweichung pro Injektionspunkt im periorbitalen Bereich bei Patienten mit Spasmus facialis. 89

Abbildung 37: Mittlere Dosis mit Standardabweichung pro Injektionspunkt im perioralen und Platysmabereich bei Patienten mit Spasmus facialis 90

Abbildung 38: Mittlere Dosis mit Standardabweichung pro Injektionspunkt im periorbitalen Bereich bei Patienten mit Synkinesien 
Abbildung 39: Mittlere Dosis mit Standardabweichung pro Injektionspunkt im perioralen und Platysmabereich bei Patienten mit Synkinesien.. 


\section{Tabellenverzeichnis}

Tabelle 1: $\quad$ Differenzialdiagnose des pathologischen Lidschlusses..................................5

Tabelle 2: Injektionsorte mit dazugehörigen Muskeln und deren Funktionen (modifiziert nach Grablowitz 2004, Seite 118) ........................................... 29

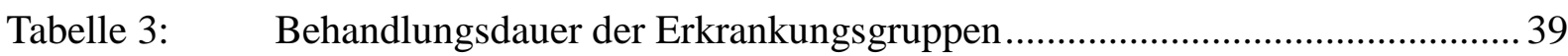

Tabelle 4: $\quad$ Zeitintervall bis zur notwendigen Wiederbehandlung..................................... 40

Tabelle 5: Ergebnisse in Form des p-Wertes der ANOVA mit Messwiederholungen, wobei die Injektionszeitpunkte 1 und 10

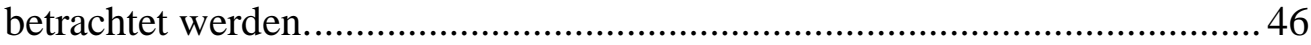

Tabelle 6: Paarvergleiche der Erkrankungsgruppen aufgetrennt nach den zwei Injektionszeitpunkten.

Tabelle 7: Ergebnisse in Form des p-Wertes der ANOVA mit Messwiederholungen, wobei die Injektionszeitpunkte 1 und 20 betrachtet werden

Tabelle 8: Paarvergleiche der Erkrankungsgruppen aufgetrennt nach dem Injektionszeitpunkt.

Tabelle 9: Ergebnisse in Form des p-Wertes der ANOVA mit Messwiederholungen, wobei die Injektionszeitpunkte 1 und 30 betrachtet werden 50

Tabelle 10: Paarvergleiche der Erkrankungsgruppen aufgetrennt nach dem Injektionszeitpunkt.

Tabelle 11: Ergebnisse in Form des p-Wertes der ANOVA mit Messwiederholungen, wobei die Injektionszeitpunkte 1 und 40 betrachtet werden

Tabelle 12: Paarvergleiche der Erkrankungsgruppen aufgetrennt nach dem Injektionszeitpunkt.

Tabelle 13: Anstieg der Injektionspunkte im Laufe der Behandlungsjahre. 55

Tabelle 14: Ergebnisse in Form des p-Wertes der ANOVA mit Messwiederholungen, wobei die Zeitpunkte 1 und 10 betrachtet werden. .... 57 
Tabelle 15: Mittelwert der Anzahl der Injektionspunkte (Standardabweichung) in den Behandlungsgruppen zum Zeitpunkt der ersten und zehnten Behandlung und deren Signifikanz.

Tabelle 16: Ergebnisse in Form des p-Wertes der ANOVA mit Messwiederholungen, wobei die Zeitpunkte 1 und 20 betrachtet werden.

Tabelle 17: Mittelwert der Anzahl der Injektionspunkte (Standardabweichung) in den Behandlungsgruppen zum Zeitpunkt der ersten und zwanzigsten Behandlung und deren Signifikanz. 60

Tabelle 18: Ergebnisse in Form des p-Wertes der ANOVA mit Messwiederholungen, wobei die Zeitpunkte 1 und 30 betrachtet werden. .... 61

Tabelle 19: Mittelwert der Anzahl der Injektionspunkte (Standardabweichung) in den Behandlungsgruppen zum Zeitpunkt der 1. und der 30. Behandlung und deren Signifikanz 62

Tabelle 20: Ergebnisse in Form des p-Wertes von der ANOVA mit Messwiederholungen, wobei die Zeitpunkte 1 und 40 betrachtet werden. .... 63

Tabelle 21: Mittelwert der Anzahl der Injektionspunkte (Standardabweichung) in den Behandlungsgruppen zum Zeitpunkt der ersten und der vierzigsten Behandlung und deren Signifikanz.

Tabelle 22: Nebenwirkungsprofil in \%-Angaben bei Patienten mit Blepharospasmus ( $n=690)$, Spasmus facialis $(n=692)$, mit Synkinesien ( $n=480)$ und bei allen Gruppen zusammen (insgesamt $n=1862) \ldots \ldots \ldots \ldots \ldots \ldots . . . . . .66$

Tabelle 23: Verlauf der mittleren Dosis pro Injektionspunkt beim Blepharospasmus ...... 88

Tabelle 24: Verlauf der mittleren Dosis pro Injektionspunkt bei Spasmus facialis............91

Tabelle 25: Verlauf der mittleren Dosis pro Injektionspunkt bei Synkinesien .................. 94 


\section{Abkürzungsverzeichnis}

\begin{tabular}{|c|c|}
\hline Ach & Acetylcholin \\
\hline BoNT & Botulinumneurotoxin \\
\hline BTX & Botulinumtoxin \\
\hline bzw. & beziehungsweise \\
\hline $\mathrm{Ca}$ & Calcium \\
\hline $\mathrm{E}$ & Einheit \\
\hline G & Gage \\
\hline GABA & Gammaaminobuttersäure \\
\hline i.m. & intramuskulär \\
\hline i.v. & intravenös \\
\hline $\mathrm{kDa}$ & Kilo-Dalton \\
\hline $\mathrm{kg}$ & Kilogramm \\
\hline KHBW & Kleinhirnbrückenwinkel \\
\hline LD 50 & letale Dosis 50\% \\
\hline M. & Musculus \\
\hline N. & Nervus \\
\hline $\mathrm{n}$ & Anzahl \\
\hline Pat. & Patient \\
\hline SD & Standardabweichung \\
\hline SNAP-25 & synaptosomal-associated protein of $25 \mathrm{kDa}$ \\
\hline SNARE & soluble N-ethylmaleimide sensitive factor attachment protein Receptor \\
\hline sog. & sogenannt \\
\hline $\mathrm{U}$ & Einheit (Unit) \\
\hline VAMP & vesicle-associated membrane protein (Synaptobrevin) \\
\hline vs. & versus \\
\hline
\end{tabular}




\section{Einleitung}

\subsection{Anatomie und Innervation der Gesichtsmuskulatur}

Genaue Kenntnis über die anatomischen Verhältnisse der Gesichtsmuskulatur ist die Voraussetzung für eine adäquate lokale Behandlung mit Botulinumtoxin. Die Gesichtsmuskeln (siehe Abbildung 1) bewegen die Lippen, Augenlider und Nasenflügel. Es handelt sich um Hautmuskeln, welche mit der Haut verwachsen sind und diese bei der Mimik bewegen. Die Änderung von Muskelkontraktionen und Muskeltonus der mimischen Muskulatur führt somit zu verschiedenen Gesichtsausdrücken. Die mimische Muskulatur wird motorisch vom Nervus facialis innerviert.

Der Hautmuskel des Halses ist das Platysma. Die mimischen Muskeln der Lidspalte sind die Musculi orbicularis oculi und corrugator supercilii. Zu den Muskeln der Mundregion gehören die Musculi orbicularis oris, buccinator, depressor labii inferioris, mentalis, depressor anguli oris, risorius, levator anguli oris, zygomaticus major et minor, levator labii superioris alaeque nasi und nasalis. Die Muskeln des Schädeldaches sind die Musculi occipitofrontalis und temporoparietalis.

Die wesentliche Aufgabe der zuvor genannten Muskeln bzw. Muskelgruppen ist die mimische Funktion. Jede Muskelgruppe kann gewisse Ausdrucksweisen auslösen. Affekte wie Angst, Wut, Ekel, Freude, Trauer und Leid werden durch Bewegung und Spannung der mimischen Muskeln und der mit ihnen verbundenen Gesichtshaut vermittelt.

Die unwillkürlichen und pathologischen Muskelbewegungen des Gesichts stigmatisieren die Betroffenen. Diese Tatsache kann dazu führen, dass sich die Betroffenen unverstanden und verunsichert aus der Gesellschaft zurückziehen. Die betroffenen Muskeln bzw. Muskelgruppen können bei verschiedenen Erkrankungen beteiligt sein. 


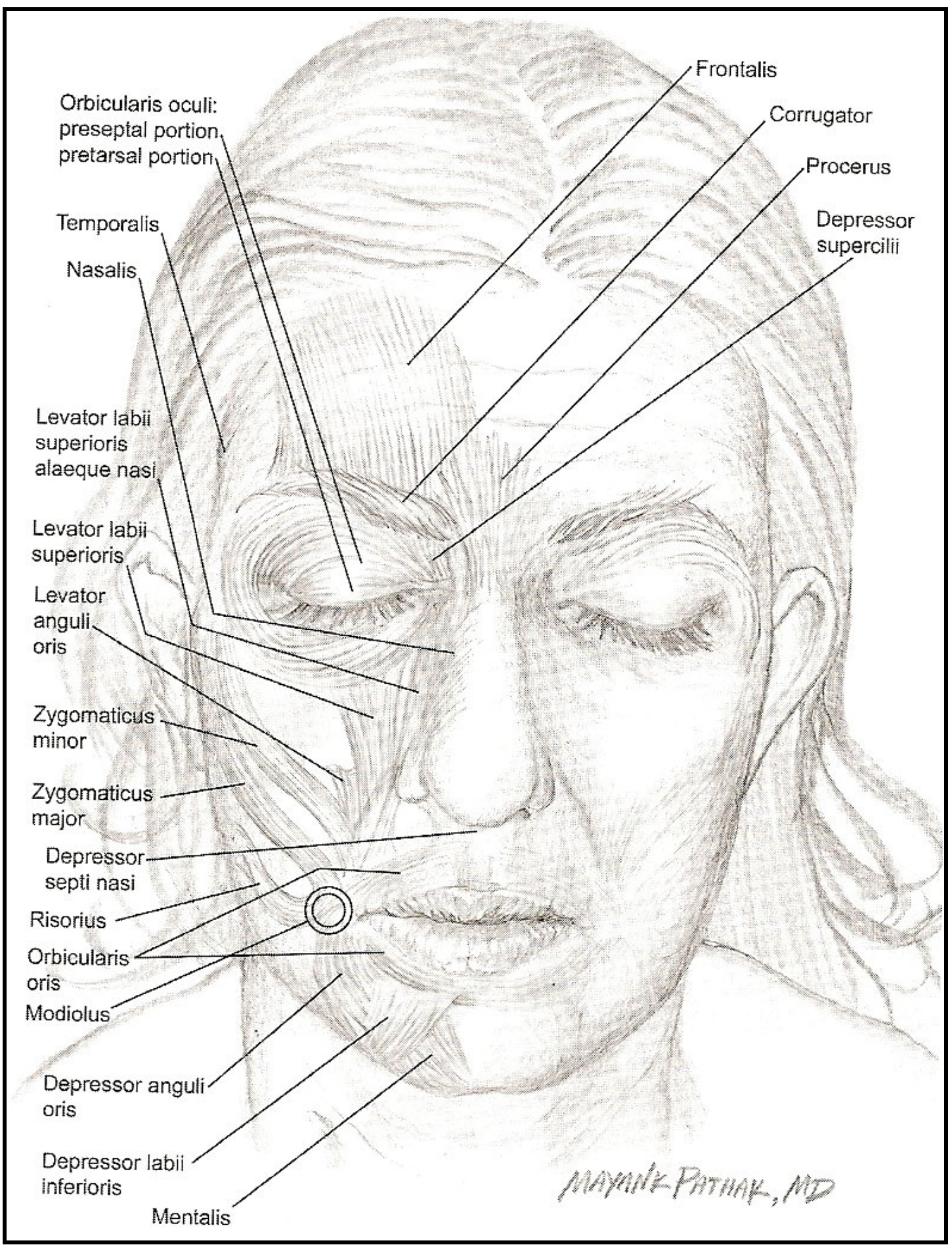

Abbildung 1: Anatomie der mimischen Muskulatur (aus Glaser 2009, Seite 134) 


\subsection{Relevante Krankheitsbilder}

\subsubsection{Klinik des Blepharospasmus}

Man unterscheidet eine primäre und sekundäre Form des Blepharospasmus. Beim essentiellen (primären) Blepharospasmus handelt es sich um eine fokale Dystonie mit Beteiligung der Muskeln der Augenlider und des Stimbereiches (Musculi procerus und corrugator). Dabei kommt es zeitweise oder ständig zu einer unwillkürlichen Kontraktion des Musculus orbicularis oculi (prätarsal, präseptal und periorbital) mit einer erhöhten Frequenz eines betont krampfartigen Lidschlusses (siehe Abbildung 2). Die Symptome treten in den meisten Fällen beidseitig auf. Diese können jedoch rechts und links unterschiedlich stark ausgeprägt sein. Die Verkrampfungen können an Intensität und Häufigkeit im Laufe der Zeit zunehmen. Im Alltag sind die Patienten daher stark behindert, denn sie können nur eingeschränkt lesen, fernsehen und Hausarbeiten erledigen. In besonders gravierenden Fällen kann es zu einer funktionellen Blindheit kommen (Jost und Kohl 2001, Kenney und Jankovic 2008, Coscarelli 2010).

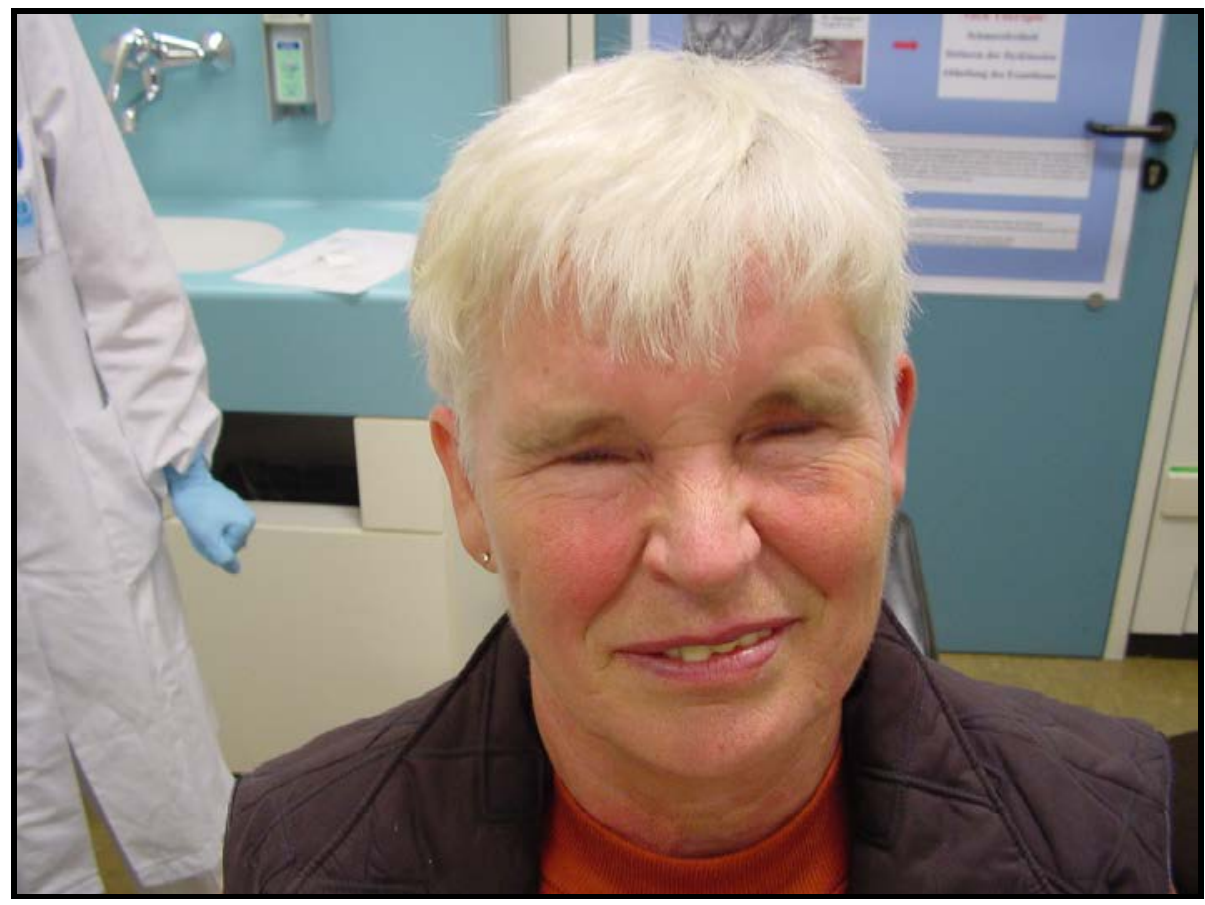

Abbildung 2: Beispiel einer Patientin mit einem Blepharospasmus. An diesem Beispiel ist zu erkennen, dass beide Musculi orbicularis oculi stark innerviert werden. Dadurch kommt es zu einem kompletten Augenschluss und einer vorüber-gehenden funktionellen Blindheit. 
Diese Beschwerden können auch weitere Muskeln des Gesichtes betreffen. Bei einigen Patienten besteht das sogenannte „Meige-Syndrom“ bzw. eine „oromandibuläre Dystonie“. Es beinhaltet unwillkürliche Kontraktionen der Muskulatur im oromandibulären Bereich in Assoziation mit einem Blepharospasmus. Es kann zu unwillkürlichen Gesichtsgrimassen, Stirnkontraktionen, einem Torticollis und zu einer spasmodischen Dysphonie kommen. Ein Blepharospasmus kann als erstes Symptom auftreten, aber auch erst der oromandibulären, laryngealen oder pharyngealen Muskulatur folgen. Bei diesem Erkrankungsbild sind auch andere Hirnnerven mitbetroffen, insbesondere die Nervi trigeminus, vagus und hypoglossus (Mauriello et al. 1996a). Es existiert auch eine Sonderform des essentiellen Blepharospasmus, der sog. „Levator-Inhibitionstyp“ oder der „Blepharospasmus vom palpebralen Typ“. Bei dieser Form fehlen die typischen starken Verkrampfungen, trotzdem ist der Patient nicht in der Lage die Augen zu öffnen. Als Ursache ist hier die Insuffizienz der Funktion des Musculus levator palpebrae anzusehen (Roggenkämper und Laskawi 2004).

Die Ätiologie des Blepharospasmus ist unklar. Organische Ursachen für das Auftreten wurden von Jankovic und Patel (1983) beschrieben. Ein Großteil der Spasmen kann ätiologisch jedoch nicht genau entschlüsselt werden. Man geht davon aus, dass eine anormale Funktion der Basalganglien zu den unwillkürlichen Verkrampfungen führt (Vitek 2002).

Die Diagnose des essentiellen Blepharospasmus ergibt sich hauptsächlich aus der Klinik. Im Idealfall sind die störenden Verkrampfungen beim Arztbesuch zu sehen. Häufig sind sie jedoch auch nicht zu beobachten. Der Patient berichtet dann über Augenlidkrämpfe, aber auch über brennende, schwere Lider. Diese Symptome sind typischerweise im Liegen nicht vorhanden und treten häufig situationsabhängig auf. Sie können z.B. durch Stress, intensiven Sonnenschein, Wind, Fernsehen oder Lesen provoziert werden.

Es gibt mannigfaltige Ursachen, die zum unwillkürlichen Lidschluss führen können. Eine ophthalmologische und neurologische Untersuchung des Patienten sollte daher durchgeführt werden. Die Differenzialdiagnosen des Blepharospasmus sind vielfältig und sollten sorgfältig abgewogen werden (siehe Tabelle 1). Für die Diagnosefindung ist die Computertomographie und Kernspintomographie den unklaren Fällen vorbehalten. Die Sonderform des Blepharospasmus, der „Levator-Inhibitionstyp“, kann auf Grund einer ähnlichen Klinik leicht mit einer Myasthenie verwechselt werden. Die Differenzialdiagnostik umfasst den Simpson-Test, das Lidtwitch-Zeichen, Antikörperbestimmungen und elektromyographische Ableitungen (Roggenkämper und Laskawi 2004). 


\begin{tabular}{|c|c|}
\hline Ursache & Erkrankung \\
\hline $\begin{array}{l}\text { Schwäche des } \\
\text { Levator palpebrae }\end{array}$ & $\begin{array}{l}\text { Ptosis } \\
\text { (verschiedene Ursachen) }\end{array}$ \\
\hline $\begin{array}{l}\text { Unwillkürliche } \\
\text { Kontraktionen des M. } \\
\text { orbicularis oculi }\end{array}$ & $\begin{array}{l}\text { Zentrales Nervensystem } \\
\text { Dystonie (essentieller Blepharospasmus) } \\
\text { Ticks } \\
\text { Tardive Dyskinesie } \\
\text { Chorea } \\
\text { Myoklonus } \\
\text { Tetanus } \\
\text { Anfallsleiden } \\
\text { Blickrichtungsabhängiger Blepharoklonus } \\
\text { Peripheres Nervensystem } \\
\text { Spasmus hemifacialis } \\
\text { Faziale Synkinesien } \\
\text { Faziale Myokymie } \\
\text { Tetanie } \\
\text { Muskelerkrankungen } \\
\text { - Myotonie }\end{array}$ \\
\hline Augenerkrankungen & $\begin{array}{l}\text { Erkrankungen der Bindehaut, Hornhaut, Entzündung } \\
\text { der Iris und Uvea }\end{array}$ \\
\hline Sonstige & $\begin{array}{l}\text { - „Apraxie der Lidöffnung“ } \\
\text { Parkinsonismus } \\
\text { Nicht neurologisch oder ophthalmologisch bedingt } \\
\text { Schlaf } \\
\text { Willkürlicher Lidschluss } \\
\text { Psychogener Lidschluss }\end{array}$ \\
\hline
\end{tabular}

Tabelle 1: Differenzialdiagnose des pathologischen Lidschlusses. (modifiziert nach Roggenkämper und Laskawi 2004, Seite 76) 
Die Prävalenz des Blepharospasmus beträgt 1: 10 000. Somit handelt es sich um eine seltene Erkrankung, welche die betroffenen Patienten jedoch stark stigmatisiert. Die Erkrankung betrifft meist Patienten im mittleren bis hohen Alter. Es erkranken häufiger Frauen als Männer über dem 56. Lebensjahr (Coscarelli 2010, Peckham et al. 2011). Die Patienten werden häufig nicht verstanden und als psychisch krank „abgestempelt“. Sie ziehen sich immer mehr aus dem öffentlichen Leben zurück. Es ist bekannt, dass diese Erkrankung häufig mit Depressionen, Angst und einer geringen Lebensqualität einhergeht (Pekmezovic et al. 2009). Inzwischen gibt es jedoch Selbsthilfegruppen. Die bekannteste ist die Deutsche Dystonie Gesellschaft (DDG). Dies ermöglicht den Betroffenen die gegenseitige Begegnung und praktische Hilfe auch beim Werben für mehr Verständnis unter ihren Mitmenschen.

\subsubsection{Klinik des Spasmus facialis}

Das Krankheitsbild des Spasmus facialis ist charakterisiert durch das Auftreten tonischklonischer Krämpfe der mimischen Muskulatur (siehe Abbildung 3). Diese Spasmen beginnen häufig im Bereich des Musculus orbicularis oculi. Im Laufe der Zeit können sich die unwillkürlichen Bewegungen nach kaudal ausbreiten und die Musculi nasalis, zygomaticus, risorius, orbicularis oris und das Platysma betreffen. In der Regel besteht die Erkrankung einseitig (Jannetta et al. 1977, Wang und Jankovic 1998, Cannon et al. 2010). In seltenen Fällen können auch beide Gesichtshälften betroffen sein. Eine familiäre Häufung wurde ebenfalls beschrieben (Friedman et al. 1989, Coad et al. 1991, Tan und Jankovic 1999). 


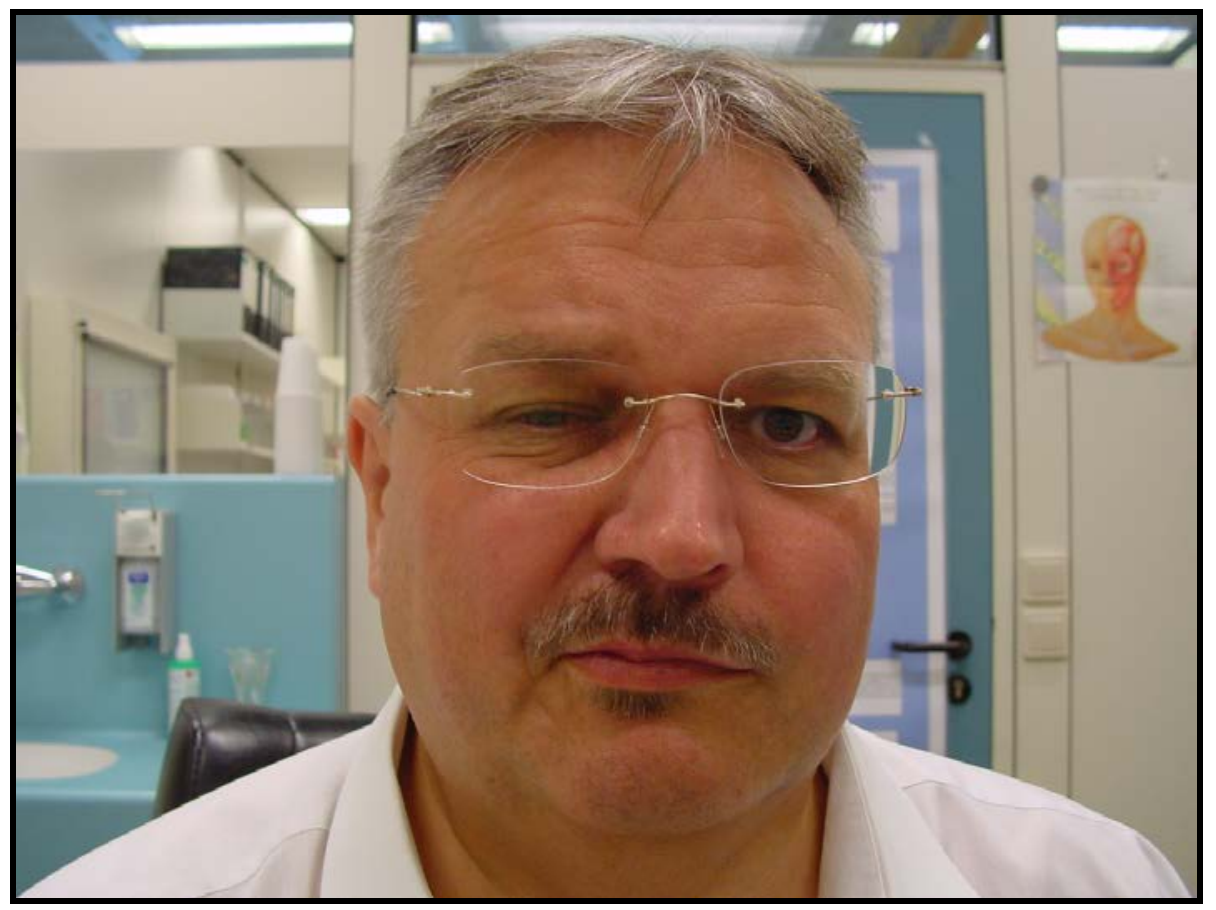

Abbildung 3: Beispiel eines Patienten mit einem Spasmus facialis.

Mann erkennt die volle klinische Ausprägung der Symptomatik. Es zeigt sich eine rechtsseitige Verkrampfung der gesamten mimischen Muskulatur.

Die Krämpfe können durch bestimmte Situationen wie psychische Erregungen, Licht, verschiedene Arten von Stress getriggert werden. Die Beschwerden können auch während des Schlafes auftreten. Der Spasmus hemifacialis betrifft häufiger Frauen als Männer. Das mittlere Alter liegt bei ca. 45 Jahren (Laskawi 2004, Frei und Roggenkämper 2009, Cannon et al. 2010).

Als häufigste Ursache eines Spasmus facialis wird eine vaskuläre Kompression (durch die Arteria cerebelli anterior inferior oder die Arteria cerebelli posterior inferior, A. basilaris, A. vertebralis) des Nervus facialis an dessen Austrittspunkt aus dem Hirnstamm, in der sogenannten „root exit zone“, einem wenig myelinisierten Bereich des Nerven, beschrieben (Campbell und Keedy 1947, Jannetta et al. 1977, Nielsen 1985, Frei und Roggenkämper 2009). Die Spasmen können jedoch auch durch Raumforderungen, die in enger topographischer Beziehung zum Nervus facialis stehen, ausgelöst werden. Dazu gehören zum Beispiel arteriovenöse Malformationen, Aneurysmen, Neurinome, Meningeome und Tumore im Bereich 
des Kleinhirnbrückenwinkels (Nagata et al. 1992, Galvez-Jimenez et al. 2001, Frei und Roggenkämper 2009).

Die Diagnose des Spasmus facialis ist bei voller Ausprägung der Symptomatik am klinischen Bild gut zu stellen. Es existieren jedoch andere Erkrankungen, die vor allem beim Beginn der Symptomatik im Augenbereich differenzialdiagnostisch abgegrenzt werden müssen. Dazu gehören beispielsweise der unilaterale Blepharospasmus, Synkinesien nach einer Fazialisdefektheilung und abgelaufene Lähmungen des Nervus facialis durch Traumen oder chirurgische Maßnahmen (siehe Tabelle 1).

Im Rahmen der Diagnostik benötigt man eine Magnetresonanztomographie zum Ausschluss einer Raumforderung oder einer Gefäßanomalie im zentralen Nervensystem. Außerdem muss eine genaue Untersuchung der Glandula parotis beidseits zum Ausschluss eines Tumors durchgeführt werden. In wenigen Fällen wurde hier die Ursache eines Spasmus facialis gefunden (Laskawi 2004).

\subsubsection{Klinik der Synkinesien nach Fazialisdefektheilung}

Synkinesien werden als unwillkürliche Mitbewegungen bestimmter Bereiche der mimischen Muskulatur beschrieben, die eine gewollte Bewegung anderer Gesichtspartien begleiten. Zum Beispiel wird beim Lidschluss der Mund bewegt oder umgekehrt, das bedeutet, bei Mundbewegungen kann es zum Lidschluss kommen (Beispiel siehe Abbildung 4).

Diese unbeabsichtigten Bewegungen der verschiedenen Gesichtspartien beeinflussen im täglichen Leben das Trinken, das Essen, das Sprechen, das Lachen und stören die Symmetrie des Gesichtes.

Mitbewegungen können ein unterschiedliches Ausprägungsmuster zeigen. Auch die Verteilung der pathologischen Mitbewegungen kann unterschiedlich ausfallen. Die Patienten sind stigmatisiert, erleiden Depressionen und erleben häufig krankheitsbedingt negative Folgen bis hin zu einer Isolation in ihrem sozialen Umfeld (Roggenkämper et al. 1990, Neely und Neufeld 1996, Laskawi und Roggenkämper 2004, Husseman und Mehta 2008, Beurskens et al. 2010). 


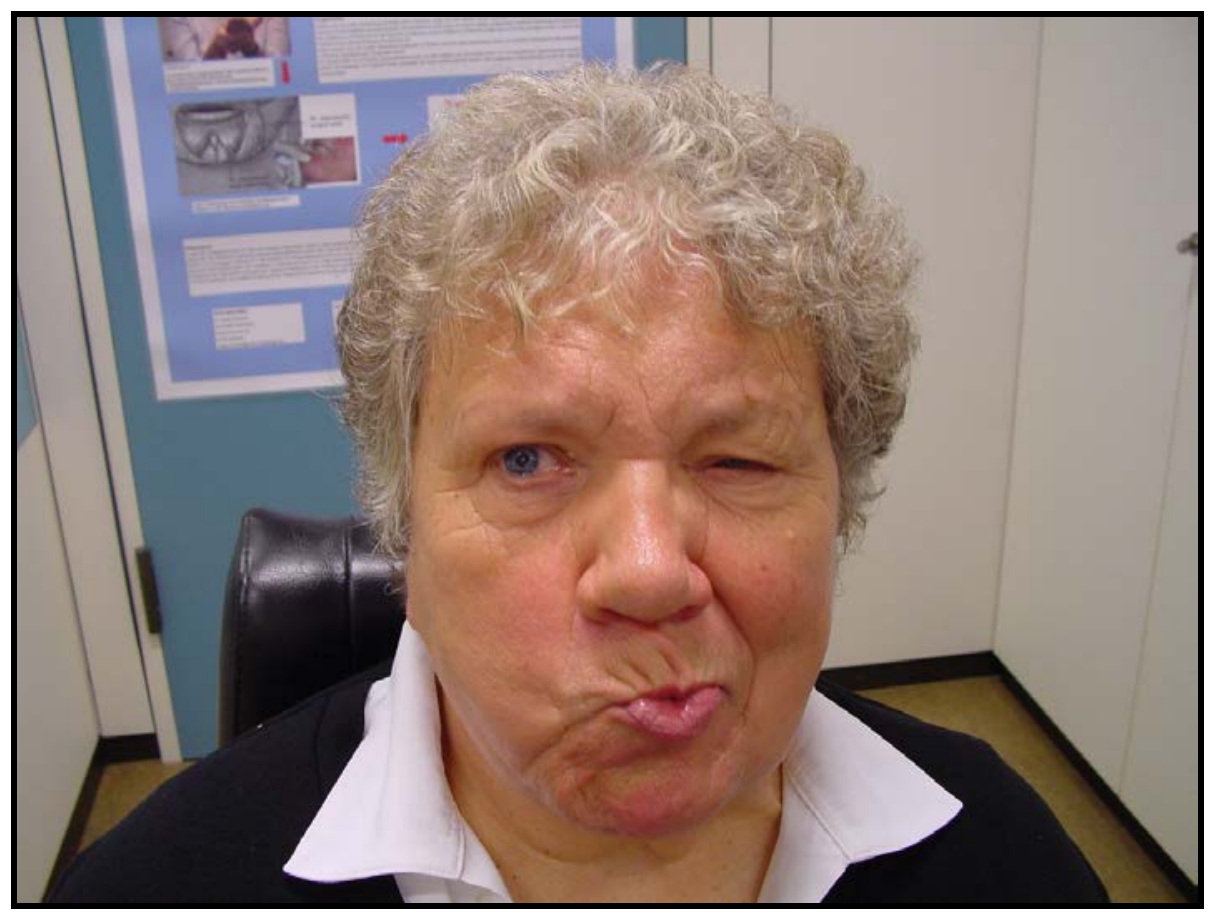

Abbildung 4: Beispiel einer Patientin mit Synkinesien.

Die Patientin wurde aufgefordert, den Mund zu spitzen.

Man erkennt eine Mitbewegung der linksseitigen periokulären Muskulatur und dadurch eine deutlich sichtbare Verengung der Lidspaltenweite. Außerdem kommt es zu einer störenden und sichtbaren Asymmetrie des Gesichtes.

Die Ursache für die Synkinesien ist in einer Nervus-facialis-Defektheilung zu finden. Die Gründe für diese Defektheilung können verschiedener Natur sein, z.B. können sie nach einer idiopathischen Fazialislähmung mit degenerativem Verlauf, nach einer Herpes-zoster-Erkrankung mit anschließender Parese oder nach rekonstruktiven Eingriffen am Nervus facialis (Hypoglossus-Fazialis-Anastomose) auftreten. Eine Abgrenzung zu anderen Erkrankungen sollte auch hier genau überlegt und durchgeführt werden (siehe Tabelle 1).

Nach einer Hypoglossus-Fazialis-Anastomose kann es zu peripheren Fehlaussprossungen von regenerierenden Axonen kommen, dadurch fehlt die „Punkt-zu-Punkt-Zuordnung“ zwischen den kortikalen motorischen Feldern und der peripheren Muskulatur (Roggenkämper et al. 1990, Laskawi und Roggenkämper 2004). 
Das klinische Bild und eine vorausgegangene Fazialisläsion sind maßgebend für die Diagnose der Synkinesien. Bei der klinischen Untersuchung sollten bestimmte Standardbewegungen getestet werden. Dazu gehören Stirnrunzeln, Augenzukneifen, Zähnezeigen, Naserümpfen und Mundspitzen. Dabei sollten die pathologisch synchronaktiven Abschnitte der mimischen Muskulatur beobachtet werden.

\subsubsection{Zur Therapie fazialer Dyskinesien}

In der Anfangszeit wurden beim essentiellen Blepharospasmus und Spasmus facialis sowohl eine medikamentöse und operative Therapie als auch Psychotherapie, Hypnose und Akupunktur angewandt. Unter den Arzneimitteln befanden sich Anticholinergika, Dopaminantagonisten und GABA-Agonisten (z.B. Baclofen und Clonazepam). Die Medikamente verursachten zum Teil erhebliche Nebenwirkungen wie Müdigkeit, Mundtrockenheit und Halluzinationen. Zu den operativen Verfahren zählen unter anderem eine direkte Ausschaltung von Muskelfasern des Musculus orbicularis oculi, die Elektrokoagulation, die Stimulation mit galvanischem Strom oder die Dekompressionsoperation des Nervus facialis nach der Methode von Jannetta (Jannetta et al. 1977), die heute beim Spasmus facialis die operative Methode der ersten Wahl darstellt. Für viele Patienten sind die Risiken einer solchen Operation wie eine Fazialisparese, ein einseitiger Hörverlust, intrakranielle Blutungen oder ein Schlaganfall ein zu hohes Risiko. Bestimmte Autoren haben auch zeigen können, dass einige Patienten von den angewandten Verfahren nur ungenügend profitiert haben (Lovely et al. 1998, Calace et al. 2003, Huh et al. 2008). Die mikrovaskuläre Dekompressionsoperation ist gemäß der aktuellen Literatur aber weiterhin als die Methode der Wahl unter den verschiedenen operativen Verfahren anzusehen. Dabei ist eine hohe Effektivität der Methode beschrieben worden (Barker et al. 1995, Huh et al. 2008).

Zur Therapie von Synkinesien wurden außer operativen Maßnahmen (wie z.B. die selektive Neurektomie) auch physiotherapeutische Trainingsmethoden beschrieben. Bei einem Teil der Patienten brachte ein Biofeedbacktraining, teilweise mit einer akustischen oder elektrophysiologischen Kontrolle, eine Besserung der Symptomatik.

Nach einem Rückkopplungstraining mit einem Elektromyogramm oder mit einem krankengymnastischen Übungsprogramm, der sog. MIME-Therapie, soll ein besseres Resultat der mimischen Muskulatur erreicht werden (Beurskens und Heymans 2004, Beurskens et al. 2006, Husseman und Mehta 2008). 
Seit mehreren Jahren steht die Therapie mit Botulinumtoxin zur Verfügung. Bei den genannten Erkrankungen erwies sich die Therapie mit Botulinumtoxin A als effektive und nebenwirkungsarme Behandlung. Die Substanz wurde zunächst zur Behandlung des Strabismus und Anfang der 80’er Jahre versuchsweise bei Lidkrämpfen (Blepharospasmus, Spasmus facialis) eingesetzt (Roggenkämper 2007). Überraschend gute Erfolge führten zu einer ausgedehnten Erforschung und zu einem breiten Einsatzbereich dieser Substanz.

\subsection{Botulinumtoxin}

\subsubsection{Clostridium botulinum}

Botulinumtoxin ist ein zelluläres Gift, das von einem grampositiven, stäbchenförmigen Bakterium unter anaeroben Bedingungen und alkalischem Milieu gebildet wird. Natürliche Rückzugsorte für das Bakterium befinden sich beispielsweise im Erdboden, im Staub oder im Meeressediment.

Dieses Gift ist hitzelabil, kann also durch hohe Temperaturen $\left(85^{\circ} \mathrm{C}\right)$, wie zum Beispiel beim Kochen, inaktiviert werden. Das Bakterium Clostridium botulinum bildet thermoresistente Sporen, die zur Vermehrung dienen und in luftabgeschlossenen oder mangelhaft konservierten Nahrungsmitteln (z.B. Konservendosen) keimen und sich vervielfältigen können (Cherington 2004, Erbguth 2008).

Der Arzt und Dichter Dr. Justinus Christian Kerner beschrieb erstmals 1817 die Symptome einer Intoxikation mit Botulinumtoxin. Daher wird diese auch als die Kerner'sche Erkrankung oder Botulismus bezeichnet (Kreyden et al 2000, Erbguth 2008).

Der Begriff Botulismus (botulus, lat. Wurst) wurde erstmals von Pierre van Ermengem, einem Mikrobiologen aus Gent, verwandt, der das verantwortliche Bakterium (damals Bacillus botulinus) 1895 erstmals isolieren konnte (Kreyden et al. 2000, Erbguth 2008). Der genaue Wirkmechanismus des Botulinumtoxins konnte jedoch erst nach dem zweiten Weltkrieg nachgewiesen werden. Erst im Jahre 1946 wurde das Botulinumtoxin A in reiner Form hergestellt. Im Jahre 1949 konnte nachgewiesen werden, dass Botulinumtoxin die Ausschüttung von Acetylcholin (ACh) an der neuromuskulären Synapse blockiert. In den 60er Jahren erforschte der amerikanische Augenarzt Dr. Alan B. Scott die Möglichkeit einer medizinischen Anwendung der Substanz beim Schielen. 1977 konnten die ersten freiwilligen Probanden 
behandelt werden und 1989 wurde das erste Medikament mit Botulinumtoxin A in den USA zugelassen (Erbguth 2008).

\subsubsection{Subtypen des Botulinumtoxins}

Bis zum heutigen Zeitpunkt sind 7 Serotypen als hochspezifische Neurotoxine beschrieben worden (A bis G). Diese werden durch verschiedene Spezies von Clostridium botulinum gebildet. Dazu zählen C. botulinum, C. butyricum und C. barati (Brunger und Rummel 2009). Humanpathogen sind die Serotypen A, B, E und wahrscheinlich auch C und F (Kumaran et al. 2008). Die Gene aller Serotypen des Botulinumtoxins sind identifiziert und sequenziert. Die Gene der Serotypen A, B, E und F sind in dem bakteriellen Genom kodiert, die Serotypen C und D wurden auf einer Bakteriophagen-DNA nachgewiesen. Bei BoNT/G befindet sich das Gen auf einem Plasmid (Campbell et al. 1993, Breit und Heckmann 2000).

Die letale Dosis (LD 50) beträgt bei Menschen zwischen 0,1-1 ng/kg Körpergewicht, was das Botulinumtoxin A auf der einen Seite zu der giftigsten bekannten Substanz macht. Auf der anderen Seite ist es dadurch ein sehr potentes Medikament und immer noch die am meisten gefürchtete Biowaffe, für welche es zurzeit kein verfügbares Antidot gibt (Breit und Heckmann 2000, Schiavo et al. 2000, Arnon et al. 2001, Montal 2009, Brunger und Rummel 2009, Kumaran et al. 2008). Eine intakte Hautbarriere und die Blut-Hirn-Schranke kann jedoch durch das Toxin nicht passiert werden (Breit und Heckmann 2000, Schiavo et al. 2000).

Die auf dem Markt befindlichen und klinisch eingesetzten BoNT/A-Präparate stammen aus Chargen biochemisch aufgereinigter Kulturüberstände. Im Handel ist BoNT/A unter den Präparate-Namen: Botox ${ }^{\circledR}$ (Firma Allergan; Irvine, Kalifornien, USA; Vertrieb durch Merz, Frankfurt), Dysport ${ }^{\circledR}$ (Ipsen-LTD., Wrexham, GB; Vertrieb durch Ipsen-Pharma, Ettlingen), Xeomin ${ }^{\circledR}$ (Merz Pharmaceuticals, Frankfurt/M, Germany) und Vistabel ${ }^{\circledR}$ (Firma Allergan Irvine, Kalifornien, USA; Vertrieb durch Merz, Frankfurt) erhältlich. Botulinumtoxin B ist unter dem Präparatnamen Neurobloc ${ }^{\circledR}$ (in Europa)/ Myobloc ${ }^{\circledR}$ (in USA) zu erhalten. Diese Substanz soll jedoch eine höhere Affinität zu den Synapsen des Autonomen Nervensystems als zu den Synapsen der motorischen Endplatte haben. Dieses führte zu einer höheren Nebenwirkungsrate im Autonomen Nervensystem. Dieser Fakt und eine sehr hohe Antigenität des Präparates führten nur zu einem begrenzten Einsatz (Dressler und Benecke 2003, Dressler und Bigalke 2004, Dressler 2008). Es existiert ebenso ein Präparat, das in China hergestellt und unter dem Handelsnamen Prosigne ${ }^{\circledR}$ verkauft wird. 
Die Indikationen und Dosisäquivalenzen der verschiedenen Präparate können nicht ohne weiteres übernommen werden (siehe hierzu Abbildung 5).

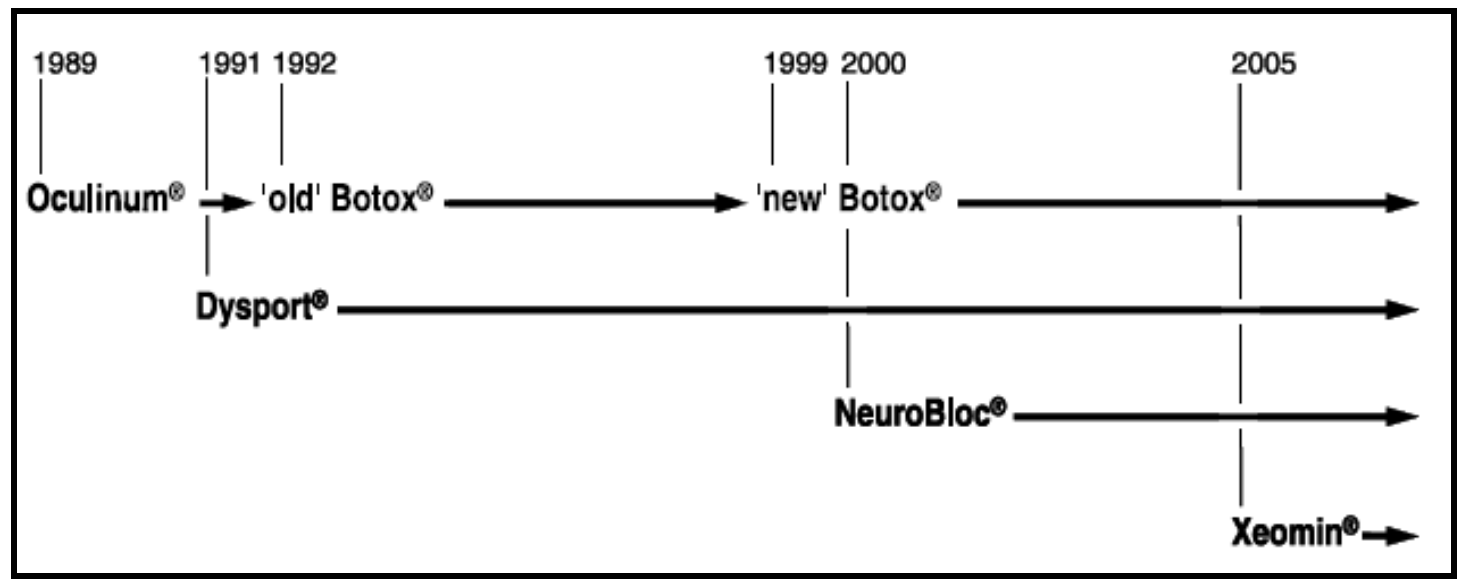

Abbildung 5: Entwicklung der Botulinumtoxin-Präparate (aus Dressler 2008, Seite 576).

Das erste Präparat erschien 1989 unter dem Namen Oculinum ${ }^{\circledR}$. Im Jahr 1992 wurde das Präparat neu „Botox®“ genannt. 1999 wurde eine neue modifizierte Formulierung des Botulinumtoxins erstellt. Das Präparat behielt jedoch den Namen Botox® ${ }^{\circledR}$. Dysport ${ }^{\circledR}$ wurde im Jahr 1991 als anderes BoNT/A Präparat registriert. Xeomin ${ }^{\circledR}$ stellt seit 2005 die neueste Formel des BoNT/A. Als BoNT/B ist seit dem Jahr 2000 das Präparat Neurobloc® zu bekommen.

Schon der oben erwähnte Justinus Kerner stellte 1822 die Hypothese auf, dass das Gift zu einem therapeutischen Zweck in Erkrankungen des Nervensystems dienen könnte. Erst sehr viele Jahre später war der Augenarzt Alan B. Scott Ende der 1970er Jahre der Erste, der Botulinumtoxin als Therapeutikum zur Behandlung des Strabismus und zum Ausgleich eines Schielwinkels verwendete (Erbguth 2008). Auf Grund der zunehmenden Kenntnisse über die Wirkung des Toxins und der Genese verschiedener Erkrankungen kam es im weiteren Verlauf weltweit zu einer Ausdehnung des Indikationsspektrums für den Einsatz von Botulinumtoxin. 


\subsubsection{Klinik des Botulismus}

Botulismus ist eine schwerwiegende neurologische Erkrankung, die durch eine schlaffe Lähmung der glatten und quergestreiften Muskulatur gekennzeichnet ist. Ausgelöst wird das Krankheitsbild durch das Toxin des Bakteriums Clostridium botulinum.

Verschiedene Untersuchungen haben gezeigt, dass die Dauer der Symptomatik je nach Subtyp des Toxins unterschiedlich ausgeprägt sein kann. Das BoNT/A, im Vergleich zu BoNT/B und BoNT/E, ruft die stärksten Beschwerden hervor, die am längsten andauern. Die neuromuskuläre Aktivität erholt sich auch im Vergleich zu BoNT/B und BoNT/E nur sehr langsam (Wochen bis Monate) (Cherington 2004).

Botulinumtoxin führt nach einer Inkubationszeit von wenigen Stunden bis Tagen zu Übelkeit, Erbrechen, Bauchschmerzen und zu einer Durchfallsymptomatik. Je kürzer die Inkubationszeit ausfällt, desto schwerer ist die Vergiftung und dementsprechend höher die Sterblichkeitsrate. Therapeutisch steht zu dieser frühen Erkrankungsphase ein Antitoxin (Botulismus-Antitoxin, Firma Behringer; polyvalentes Immunserum vom Pferd) zur Verfügung, welches eine Progression der Intoxikation wirksam verhindern kann. Gelangt das Toxin jedoch über die Blutbahn in die synaptischen Endungen der Nervenbahnen, ist die Wirkung des Antitoxins nicht mehr möglich (Sautter et al. 2001).

Kommt es zu einem Übertritt des Toxins in die Nervenendigungen, folgen im weiteren Verlauf der Vergiftung beginnende Sehstörungen, Dysphagie, Dysarthrie, Xerostomie und Hauttrockenheit. Anschließend folgt eine Paralyse der Muskulatur der oberen und unteren Extremitäten. Das Vollbild der Erkrankung bildet sich erst nach 3-7 Tagen aus und geht mit einer vollständigen Lähmung der gesamten glatten und quergestreiften Muskulatur einher.

Die daraus resultierende Klinik setzt sich aus Tetraparese, Darmatonie, Harnverhalt, Herzrhythmusstörungen und letztendlich Atemmuskellähmung zusammen. Zu diesem Zeitpunkt der Erkrankung steht als Therapiemöglichkeit nur eine intensivmedizinische Behandlung mit Beatmung zur Verfügung (Breit und Heckmann 2000, Foran et al. 2003, Cherington 2004).

Außer dem eben beschriebenen klassischen Botulismus gibt es noch andere Formen des Krankheitsbildes. Eine wichtige Form ist der Säuglingsbotulismus, der zuerst in den 1970er Jahren beschrieben wurde (Arnon et al. 1977). Es handelt sich dabei um eine gastrointestinale Infektion mit Sporen des Clostridium botulinum. Betroffen sind häufig Säuglinge bis zum 6 . Lebensmonat. Als wesentlicher Risikofaktor wird dabei das Verabreichen von Honig angesehen. 
Außerdem sind der inhalative, iatrogene und der Wundbotulismus bekannt. Der Wundbotulismus ist darunter die seltenste Form. Die Anzahl der Infektionen stieg jedoch aufgrund des weltweiten Drogenkonsums. Dabei kommt es meist bei Drogenabhängigen (i.v.Drogenabusus und intranasale Kokainaufnahme) $\mathrm{zu}$ einer Kontamination des Drogenaufnahmeortes. Es entstehen kleine mit Clostridium botulinum infiltrierte Abszesse. Das Toxin breitet sich folgend dann im gesamten Kreislaufsystem aus (Sautter et al. 2001, Cherington 2004).

Im Jahr 2004 sind 4 Fälle durch eine Vergiftung mit Botulinumtoxin A nach einem ästhetischen Einsatz gemeldet worden. Allerdings wurde eine dafür nicht zugelassene, hochdosierte Substanz verwendet (Chertow et al. 2006).

\subsubsection{Wirkmechanismus des Neurotransmitters}

Die Zielstruktur, welche klinisch relevant ist, befindet sich an der cholinergen neuromuskulären Endplatte. Die cholinerge Neurotransmission beinhaltet 6 Schritte: Synthese, Lagerung, Freisetzung, Bindung, Inaktivierung und Wiederverwertung des Neurotransmitters Acetylcholin (siehe Abbildung 6). Der Neurotransmitter wird in der präsynaptischen Nervenendigung synthetisiert und in synaptischen Vesikeln gespeichert. Nachdem ein neues Aktionspotential die synaptische Endigung der Nervenzelle erreicht hat, führt die Erhöhung der intrazellulären Kalzium-Konzentration am terminalen Nervenende zu einer Ausschüttung (Exozytose) des Acetylcholins aus den Vesikeln in den synaptischen Spalt. Die Transmittermoleküle diffundieren dann an die postsynaptische Membran, öffnen dort bestimmte Ionenkanäle, was zu einer Muskelerregung führt. Das Acetylcholin (ACh) wir durch die AChEsterasen zu Essigsäure und Cholin abgebaut. Letzteres wird durch einen hochspezifischen Mechanismus zurück in die präsynaptische Endigung aufgenommen und erneut zur Bildung von ACh verwendet (Huang et al. 2000). 


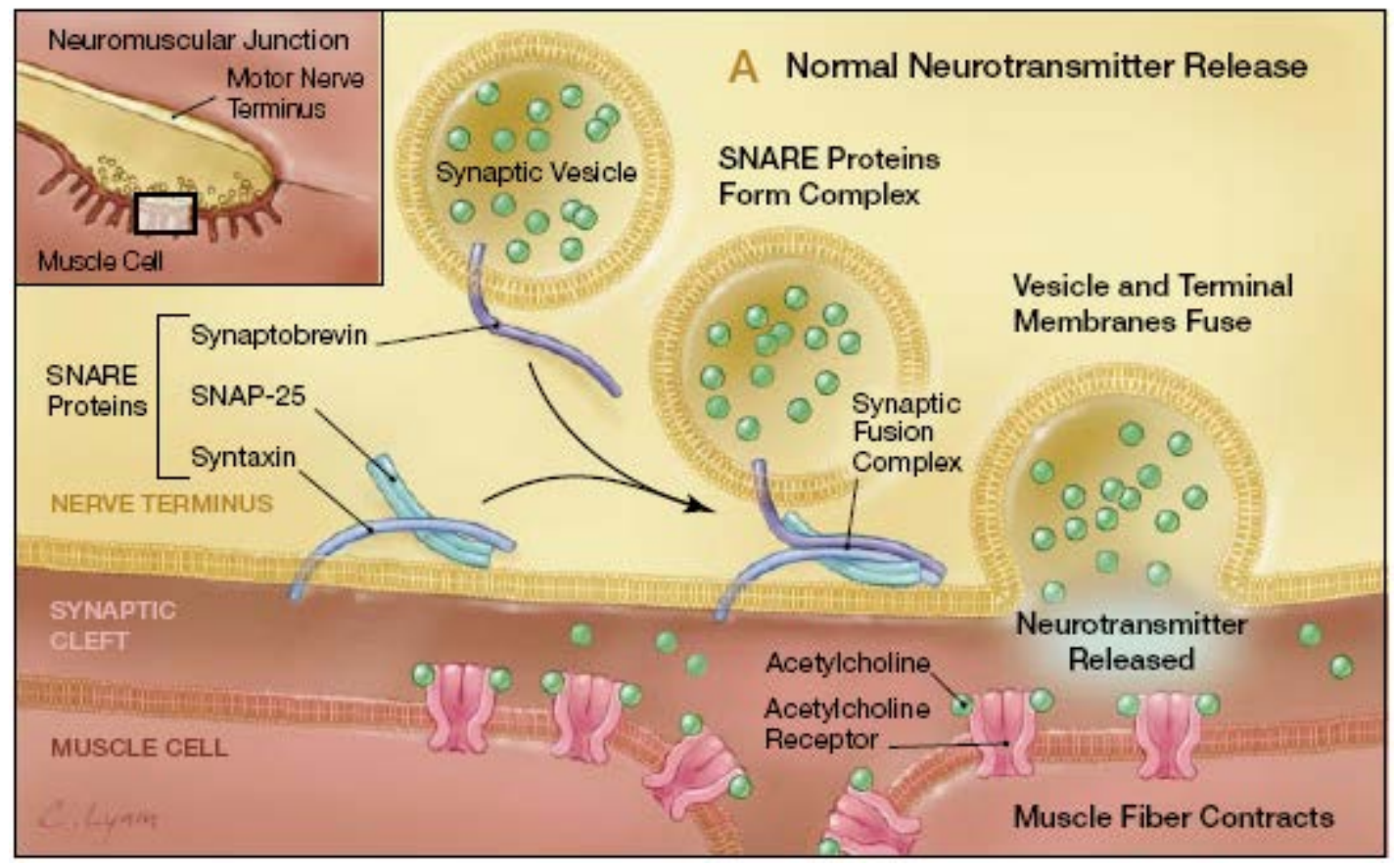

Abbildung 6: Wirkmechanismus des natürlichen Transmitters (aus Arnon et al. 2001, Seite 1061).

In der präsynaptischer Nervenendigung wird der Neurotransmitter ACh Synthetisiert und in den synaptischen Vesikel gespeichert. Nach dem Ankommen eines Aktionspotentials wird die Fusion des synaptischen Vesikels mit der präsynaptischen Nervenendigung und die Freilassung des Neurotransmitters ACh in den synaptischen Spalt an der neuromuskulären Endplatte durch einen Proteinkomplex des Exozytose-Apparates vermittelt. Der Proteinkomplex, genannt SNARE, ist zusammengesetzt aus Synaptobrevin, SNAP-25 und Syntaxin. Nach der Fusion kommt es zur Ausschüttung von ACh in den synaptischen Spalt, Bindung des Transmitters an die Rezeptoren der Muskelmembran und zu einer Erregung des Muskels.

\subsubsection{Struktur und Wirkung des Botulinumtoxins}

Sowohl das BoNT/A als auch andere Botulinumtoxin-Serotypen werden zunächst als einzelne, inaktive 150-kDa-Proteine gebildet. Anschließend werden sie proteolytisch in eine schwere (100 kDa) und eine leichte (50 kDa) Kette gespalten und über eine Disulfidbrücke miteinander 
verbunden (siehe Abbildung 7). Dieses Dichain-Molekül stellt die biologisch aktive und stabile Form des BoNT/A dar. Den Wirkmechanismus des Toxins kann man in bestimmte Einzelschritte einteilen: a)Bindung des BoNT/A an die präsynaptische Membran, b)Vesikelinternalisation durch die rezeptorvermittelte Endozytose, c)Translokation der leichten Kette aus dem Endosom in das Zytosol (siehe Abb. 8) und d)Inaktivierung des Exozytose-Apparates der ACh-Ausschüttung (siehe Abbildung 9).

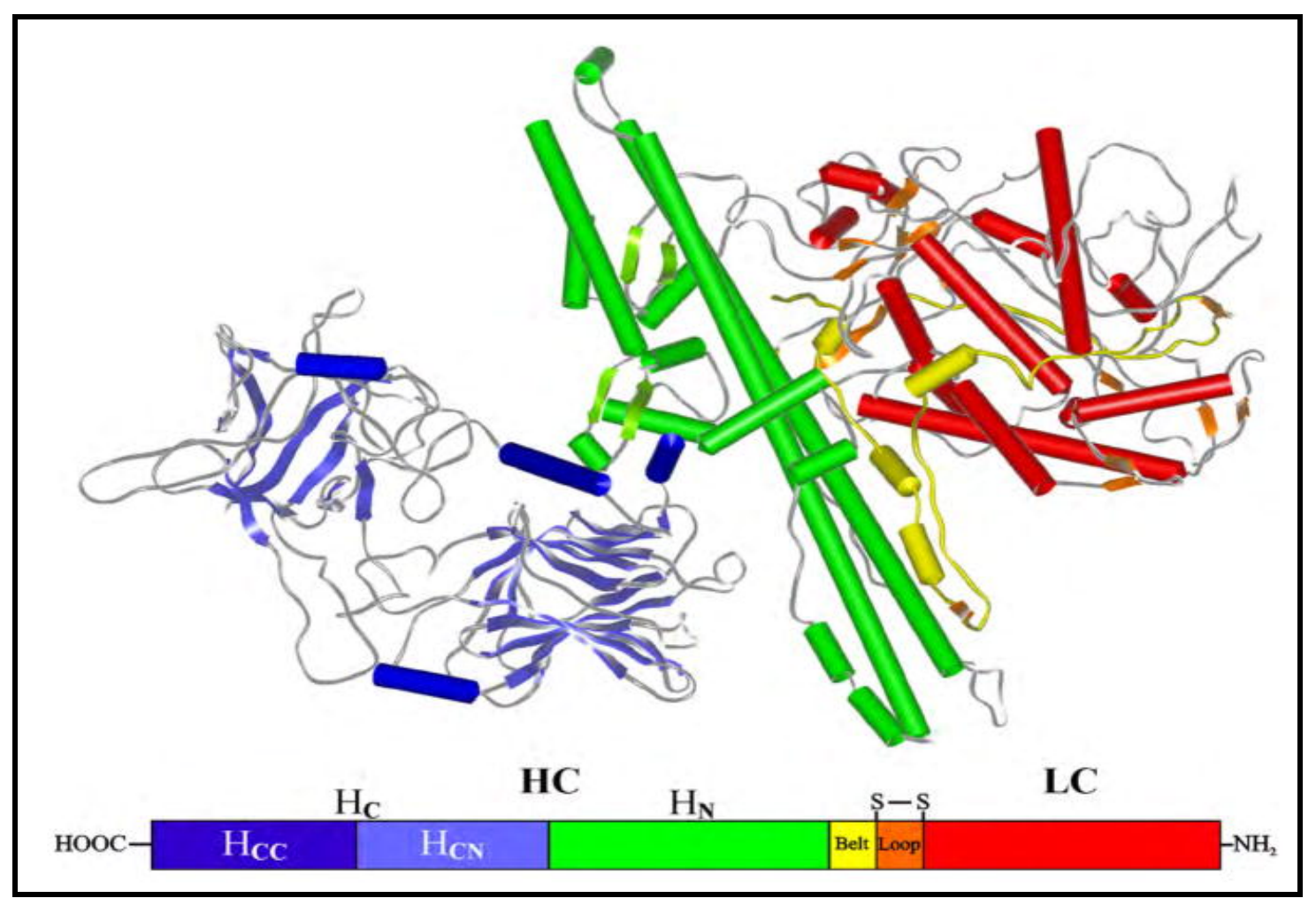

Abbildung 7: Molekülstruktur des Botulinumtoxin A (aus Brunger und Rummel 2009, Seite 551).

Darstellung der Struktur der 150-kDa-Domäne, bestehend aus einer schweren Kette (100 kDa) und eine leichten Kette (50 kDa), die durch eine Disulfidbrücke miteinander verbunden sind. 


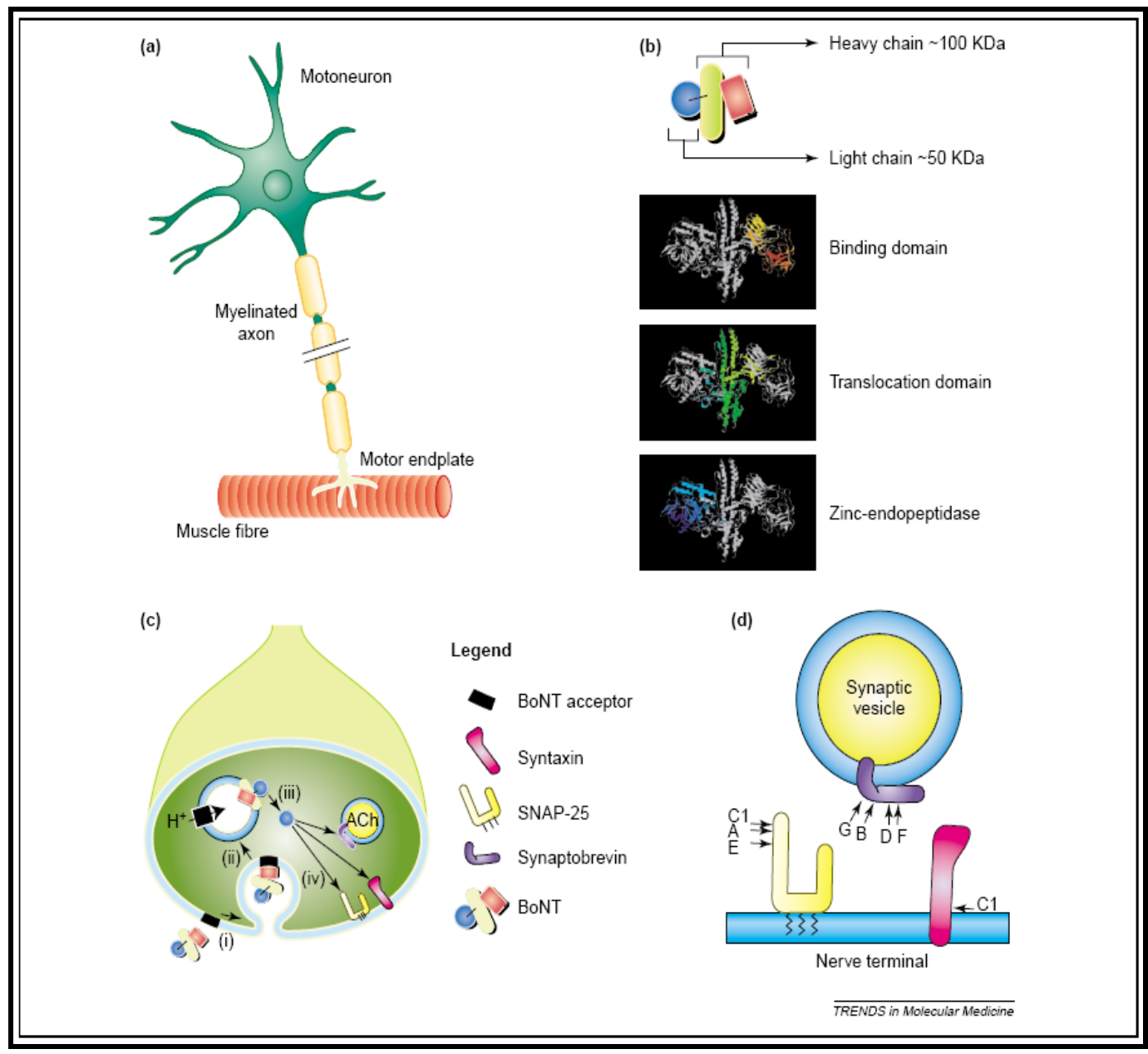

Abbildung 8: Modell für Bindung, Vesikelinternalisation und Translokation der leichten

Kette ins Zytosol durch die Plasmamembran (aus Foran et al. 2003, Seite 292).

Abb. 8a stellt die motorische Endplatte als Ziel des BoNT dar.

Abb.8b zeigt die Struktur des BoNT/A und ihre drei funktionellen Domänen. Abb. 8c: Durch die Interaktion von BoNT/A mit Gangliosiden kommt es zu einer Bindung des Substrates an die präsynaptische Membran (I). Das Einströmen von Ca stimuliert die Fusion des Vesikels mit der Membran und es kommt zur Internalisation des Vesikels in das Zytoplasma (II). Durch die ATP gesteuerte Protonenpumpe werden die Endosomen immer saurer und es kommt zur Translokation (III) und katalytischen Aktivität der leichten Kette im Zytosol (IV). 
Abb. 8d zeigt die verschiedenen SNARE Proteine des Exozytoseapparates (VAMP, SNAP-25, Syntaxin) als Endziel der verschiedenen Botulinumneurotoxine.

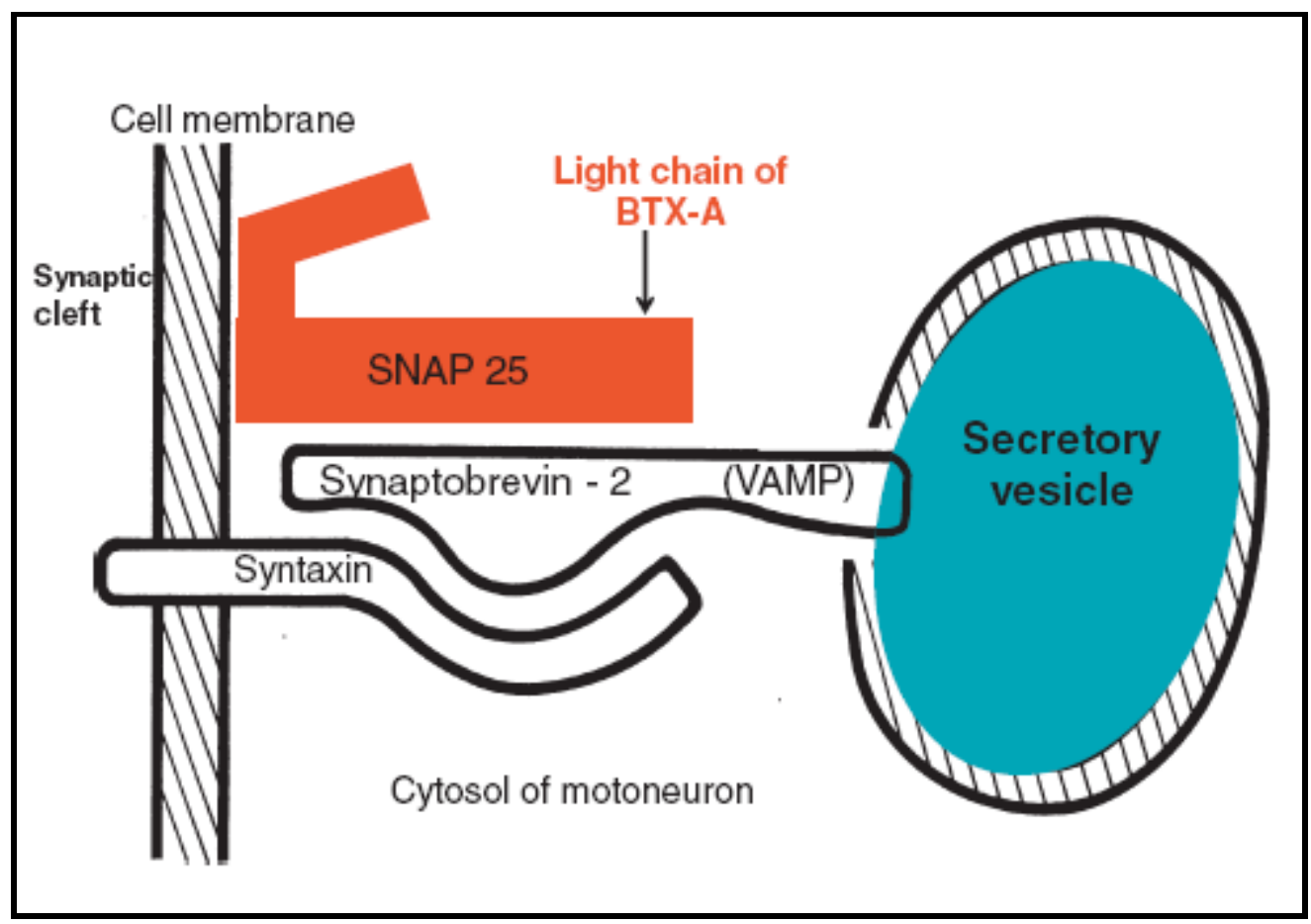

Abbildung 9: Wirkmechanismus von Botulinumtoxin A (aus Frevert 2009, Seite 12).

Die leichte Kette des BTX spaltet selektiv das Protein SNAP25, was zu dem SNARE-Komplex des Exozytose-Apparates der präsynaptischen Vesikeln gehört. Die ACh-Ausschüttung in den synaptischen Spalt wird dadurch unterbrochen.

Beiden Ketten kommen in dem Wirkmechanismus unterschiedliche Funktionen zu. Die schwere Kette ist mit dem C-terminalen Ende (Hc-Fragment, aminoterminal) verantwortlich für die Rezeptorbindung des Toxins an der Zellmembran des Motoneurons. Das Hc-Fragment bindet an neuronalen Membranstrukturen, die sogenannte Polysialoganglioside besitzen (siehe Abbildung 7). Für die Translokation durch die Zellmembran ist das N-terminale Ende (HnFragment) der schweren Kette zuständig. Durch diese rezeptorvermittelte Endozytose, stimuliert durch das ankommende Potential und Erhöhung der intrazellulären Kalzium-Konzentration, gelangt das Toxin in die Endosomen der Nervenendigungen. Durch die ATP-ge- 
steuerte Protonenpumpe kommt es zu einer Ansäuerung des Vesikelmilieus und zur Translokation des BoNT ins Zytosol. Der leichten Kette mit dem N-terminalen Ende kommt die katalytische Aufgabe als eine Zinkendopeptidase zu. Nach Spaltung der Disulfid-Brücke des Botulinumtoxins hemmt die leichte Kette selektiv ein Protein, welches SNAP-25 genannt wird. Das Protein ist involviert in einen Proteinkomplex des Exozytoseapparates der AChAusschüttung (SNARE). Der SNARE-Komplex ist integriert in die Membran der ExozytoseVesikel. Es kommt zu einer neuromuskulären Blockade durch die toxische Wirkung der leichten Kette (Schiavo et al. 2000, Grumelli et al. 2005, Fischer und Montal 2007, Kumaran et al. 2008, Brunger und Rummel 2009, Baldwin und Barbieri 2009, Frevert 2009).

Verschiedene Botulinumtoxin-Serotypen spalten unterschiedliche Anteile des SNARE-Komplexes. BoNT/A und E hemmen das Protein SNAP-25, die BoNT/B, D, F und G hemmen das Protein VAMP (Synaptobrevin-2). Das BoNT/C spaltet als einziges Toxin zwei Proteine: SNAP-25 und Syntaxin. Für die Ausschüttung von ACh ist SNAP-25 unverzichtbar (Schiavo et al. 2000, Huang et al. 2000, Foran et al. 2003, Grumelli et al. 2005, Kumaran et al. 2008). Eine signifikante Reduktion der Menge an SNAP-25 ohne Verminderung anderer synaptischer Proteine, die an dem Prozess der Exozytose beteiligt sind (Synapsin I, Synaptotagmin, Synaptophysin u. a.), konnte durch Botulinumtoxin-A-Wirkung nachgewiesen werden (Blasi et al. 1993). Es folgt eine schlaffe Paralyse der Skelettmuskulatur oder ein Ausfall der parasympathisch innervierten Organe.

\subsubsection{Regeneration der Nervenzellen}

Die enzymatische Wirkdauer der unterschiedlichen Ziel-Exozytoseproteine verschiedener Serotypen ist variabel. Die längste enzymatische Aktivität hat der Typ A, die kürzeste der Typ E. Die Wirkungsdauer der Proteinkomplexe ist auch von der Re-Syntheserate der Zielproteine abhängig. Die Nachsynthetisierung von Synaptobrevin erfolgt wesentlich schneller als die des SNAP-25. Dies erklärt die längere Wirkdauer von BTX/A gegenüber Typ B (Breit und Heckmann 2000).

Das System neuromuskuläre Synapse und die Synthese und Lagerung von ACh wird durch Botulinumtoxin nicht beeinflusst. Noch während der Blockade kommt es trotzdem zur Regeneration der Nervenzellen, einerseits durch die Neubildung von SNAP-25, andererseits durch das kompensatorische Aussprossen neuer Synapsen (sogenanntes sprouting). Schon 4 Tage nach der Injektion kommt es zu dem sogenannten sprouting und 4 Wochen nach der Be- 
handlung beginnt die Muskulatur die Arbeit langsam wieder aufzunehmen. Die neuen axonalen Fortsätze können ACh freisetzen und stellen eine neue neuromuskuläre Synapse her. Dieser Prozess ist vorübergehend, denn nach 8 Wochen übernimmt die ursprüngliche Synapse ihre Funktion wieder partiell und nach 12 Wochen komplett. Die neuen Axonsprossen bilden sich anschließend wieder vollständig zurück (de Paiva et al. 1999, Schiavo et al. 2000, Foran et al. 2003, Gill und Kraft 2010). Die Re-Synthetisierung von SNAP-25 führt zu einer erneuten Exozytose ACh-haltiger Vesikel in den synaptischen Spalt und einer folgenden Erregungsübertragung auf die postsynaptische Membran (siehe Abb. 10).

\begin{tabular}{|c|c|c|}
\hline (a) Prior to intoxication & (b) Day 2 post injection & (c) Day 14 post injection \\
\hline Twitch & Paralysis & Paralysis \\
\hline $\begin{array}{l}\text { ACh release occurs at the nerve } \\
\text { terminal. }\end{array}$ & ACh release is abolished. & $\begin{array}{l}\text { ACh release is solely detected } \\
\text { from the sprouts. }\end{array}$ \\
\hline (d) Day 30 post injection & (e) Day 60 post injection & (f) Day 90 post injection \\
\hline Twitch & Twitch & Twitch \\
\hline $\begin{array}{l}\text { ACh release occurring from } \\
\text { the sprouts mediates recovery } \\
\text { from paralysis. }\end{array}$ & $\begin{array}{l}\text { ACh release resumes in } \\
\text { parent terminals and } \\
\text { is reduced in the sprouts. }\end{array}$ & $\begin{array}{l}\text { ACh release is solely detected } \\
\text { in parent terminals. Elimination } \\
\text { of the sprouting network. }\end{array}$ \\
\hline
\end{tabular}

Abbildung 10: Regeneration der neuromuskulären Einheit (aus Foran et al. 2003, Seite 294).

a) „Vor der BTX/A Injektion kommt es zu einer Kontraktion des Muskels durch die Ausschüttung des Neurotransmitters ACh

b) Ein paar Tage nach der Injektion von BTX/A ist die Ausschüttung des ACh geblockt und der Muskel ist paretisch

c) Nach 14 Tagen ist der Muskel immer noch paretisch, es kommt jedoch zu einer ACh-Ausschüttung durch das Aussprossen neuer Synapsen ( sog. sprouting, in Gelb gezeichnet)

d) Nach 30 Tagen ist der Muskel nicht mehr paralytisch, die AChAusschüttung erfolgt nur aus den neu entstandenen Synapsen 
e) Nach 60 Tagen kommt es zu einer erneuten ACh-Ausschüttung aus der ursprünglichen Synapse und sie übernimmt nach und nach wieder ihre Aktivität

f) Nach 90 Tagen sind die neuen Axonsprossen vollständig zurückgebildet“ (Foran et al. 2003)

Die Regenerierungsprozesse und somit auch die Wirkdauer des BoNT/A halten daher durchschnittlich ca. 3 Monate an. Aus diesen Gründen sind wiederholte Behandlungen notwendig.

\subsubsection{Therapeutische Breite}

Obwohl Botulinumtoxin das stärkste bekannte Toxin ist, besitzt Botox® eine sehr große therapeutische Breite. Mit Hilfe der LD 50 (Dosis letalis 50\%) wird die therapeutische Breite beschrieben. Unter LD 50 versteht man die Dosis, bei der 50\% einer Mauspopulation versterben. Die letale Dosis des BoNT/A für Menschen, gemessen an Affenversuchen, ist geschätzt worden auf 40 U Botox i.v. je kg Körpergewicht. Die LD 50 für einen 70 kg schweren Mann, wenn man die Dosis an Primaten hochrechnet, umfasst somit den gesamten Inhalt von 30 Injektionsflaschen zu je 100 Einheiten (Botox®). Dies entspricht einer Dosis von 25003000 U (Huang et al. 2000). Die höchste verabreichte Dosis wird zur Behandlung von generalisierten Spastiken angewendet und beträgt 850 U (Dressler und Bigalke 2009).

\subsubsection{Indikationen}

In den 1970er Jahren führte die Suche nach einem Medikament, das alternativ zur konventionellen Augenmuskel-OP die Augenmuskeln reversibel schwächen kann, zu der primären Anwendung von Botulinumtoxin A durch Dr. Alan B. Scott (Roggenkämper 2007).

In Deutschland hat das Bundesinstitut für Arzneimittel und Medizinprodukte (BfArM) bisher folgende Zulassung für Botox® erteilt:

Spasmus hemifacialis, Blepharospasmus und koexistierende fokale Dystonien Zervikale Dystonie (Torticollis spasmodicus)

starke fortbestehende primäre Hyperhidrosis axillaris, die störende Auswirkung auf die Aktivitäten des täglichen Lebens hat und mit reiner topischer Behandlung nicht aus-reichend kontrolliert werden kann 
fokale Spastizität in Zusammenhang mit dynamischer Spitzfußstellung infolge von Spastizität bei gehfähigen Patienten mit infantiler Zerebralparese, die zwei Jahre oder älter sind fokale Spastizität des Handgelenks und der Hand bei erwachsenen Schlaganfall-patienten (Fachinformation Botox® 2009)

Außer den zugelassenen Indikationen existieren zahlreiche andere Anwendungsgebiete, in denen Botulinumtoxin therapeutische Erfolge erzielt hat. Diese „Off-label-Use“-Anwendungen (Therapie- oder Heilversuch mit einem zugelassenen Medikament bei nicht zugelassener Indikation) haben in verschiedenen Bereichen der Medizin ihren Stellenwert.

\subsubsection{Immunität und unerwünschte Wirkungen}

Die Behandlungsergebnisse der Botulinumtoxin-Therapie sind meist so überzeugend, dass sie für die meisten ihrer Indikationen als Therapie der Wahl gelten. Dennoch kann auch diese Therapie versagen. Ein Teil der Patienten entwickelt bei längerfristiger Anwendung eine Immunität. Bei diesem sekundären Therapieversagen konnte die Bildung neutralisierender Antikörper nachgewiesen werden. In diesen Fällen ist die Botulinumtoxin-Therapie zunächst erfolgreich, verliert jedoch ihre Wirksamkeit im späteren Verlauf der Anwendung. Von großem Interesse ist die Vermeidung der Antikörperbildung durch Botulinumtoxin. Um dieser Antikörperbildung vorzubeugen, sollten die applizierte Dosis möglichst niedrig, die Behandlungsintervalle lang sein und die so genannten Boosterinjektionen vermieden werden (Green et al. 1994, Jankovic und Schwartz 1995, Mejia et al. 2005, Dressler 2008). Bei Immunität gegen Botulinumtoxin Typ A kann eine Therapie mit Typ B eventuell durchaus erfolgreich für eine bestimmte Zeit fortgeführt werden (Aoki 2001).

Das Nebenwirkungsprofil hängt von der zu behandelnden Erkrankung und der verabreichten Dosis ab. Dies ist zum einen auf eine lokale Überdosierung und zum anderen auf eine Schwächung von Muskelgruppen zurückzuführen, welche nicht Ziel der BotulinumtoxinTherapie war. Typischerweise erscheinen die Nebenwirkungen meist innerhalb der ersten Woche und halten ca. ein bis zwei Wochen an, also deutlich kürzer als der positive therapeutische Effekt.

Lokale Komplikationen sind am häufigsten. Als typische Komplikationen bei der Behandlung im periokulären Bereich können passager eine Ptosis, Augentränen, Augenbrennen, Lagophthalmus (ggf. mit Hornhauterosionen bis hin zu einem Hornhautulkus), Doppelbilder und 
umschriebene Hämatome auftreten. Bei der Behandlung im perioralen Bereich kann es zu einem hängenden Mundwinkel, bei der Behandlung des Platysma zu Schluckbeschwerden kommen.

Über beschriebene unerwünschte Wirkungen sind die Patienten vor der Durchführung jeder Therapiesitzung ausführlich zu informieren und aufzuklären. Bei der Behandlung mit Botulinumtoxin sind auch die bekannten Kontraindikationen zu beachten. Eine Behandlung darf nicht durchgeführt werden bei Vorliegen einer Myasthenia gravis, eines Lambert-EatonSyndroms und bei bekannter Überempfindlichkeit gegen Clostridium-botulinum-Toxin Typ A. Eine unkontrollierbare Wirkungsverstärkung ist bei gleichzeitiger Einnahme von Aminoglykosidantibiotika zu erwarten. Eine relative Kontraindikation besteht bei der gleichzeitigen Einnahme von Tetrazyklinen, Lincomycin und Polymyxinen (Rohrbach und Laskawi 2004).

\subsection{Behandlung mit der Substanz Botulinumtoxin Typ A (Botox $\left.{ }^{\circledR}\right)$}

\subsubsection{Substanz}

Alle Patienten aus unserer retrospektiven Analyse wurden mit dem Präparat Botox® behandelt. Das Präparat Botox® (siehe Abb. 11; Firma Allergan) enthält 1,4 mg Trockensubstanz des Toxins. Diese 1,4 mg Toxin-Trockensubstanz entsprechen 100 Einheiten. Dies beinhaltet weniger als 5 ng Clostridium-Botulinumtoxin A. Es liegt als Toxin-Hämagglutinin-Komplex vor. Außerdem befinden sich in dem Präparat 0,5 mg Humanalbumin und 0,9 mg Natriumchlorid als Lyophilisat. Diese zusätzlichen Proteine sind für das Produkt, aber auch für den in der Natur vorkommende Botulinumtoxin-Komplex sehr wichtig. Das Hämagglutinin und Humanalbumin dienen der Stabilisierung des Komplexes und stellen somit einen Schutz vor Degeneration dar (Brin 2009).

Das Präparat Botox® wird auf Trockeneis geliefert. Das gefriergetrocknete Toxin ist bei Zimmertemperatur nur kurzzeitig haltbar. Es muss gekühlt oder tiefgekühlt (bei 2-8 ${ }^{\circ} \mathrm{C}$ oder im Tiefkühlschrank unterhalb $-5^{\circ} \mathrm{C}$ ) aufbewahrt werden. Die Substanz wird regulär mit $4 \mathrm{ml}$ physiologischer 0,9\%-iger Kochsalzlösung aufgelöst, sodass 0,1 ml Lösung ein Nanogramm des Neurotoxins enthält, dies entspricht 2,5 Einheiten (Pütz 2004). 


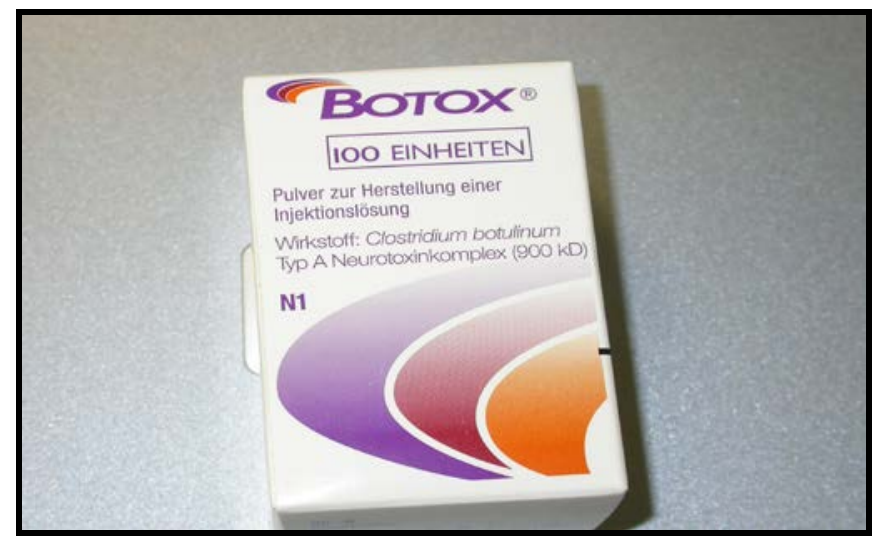

Abbildung 11: Präparat Botox ${ }^{\circledR}$ (Firma Allergan)

Die benötigte Menge wurde mittels einer 1ml-Einmalspritze (Kendall Monoject Syringe $1 \mathrm{ml}$, Northern Irland) mit 0,1 ml Einteilung entnommen. Zur Injektion wurden $27 \mathrm{G}$ EinmalInjektions-Kanülen (Sterican Braun, Melsungen) verwendet (siehe Abb.12). Nach Desinfektion der Injektionsstellen erfolgte die Behandlung entsprechend dem für den Patienten erstellten Dosierungsschema.

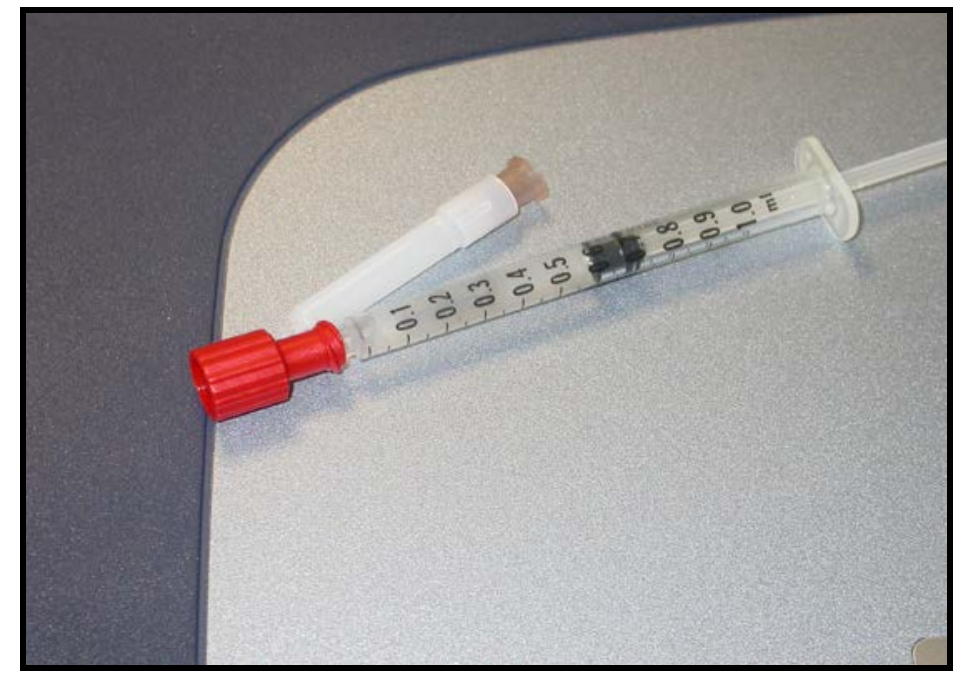

Abbildung 12: Injektionsnadel und Injektionsspritze

Durch die Unterteilung der 1-mlSpritze in 0,1-ml-Schritte sind die Standarddosierungen gut abzulesen. 0,1 ml entsprechen 2,5 E bei Auflösung der Substanz mit 4ml NaCl- Lösung. 


\subsubsection{Behandlung}

$\mathrm{Zu}$ Beginn der Behandlung wurde eine Standarddosis zwischen 1,25 und 2,5 Einheiten pro Injektionsstelle gewählt. Die Kriterien für die Wahl der Dosis, sowohl bei der ersten als auch bei den weiteren folgenden Behandlungen, waren die subjektiven Beschwerden des Patienten, das klinische Bild der Erkrankung und das Nebenwirkungsprofil. Nach diesen Anhaltspunkten wurden auch die Injektionsstellen während der ersten und den folgenden Behandlungen ausgewählt. Zur Dokumentation sowohl der Einzelpunktdosis als auch der Gesamtdosis und Lokalisation der Injektionsstellen diente der dafür speziell entwickelte Behandlungsbogen. Die gesetzten Injektionspunkte wurden als Dosissymbole für jede Injektionsstelle einzeln eingetragen (siehe Abbildung 32 im Anhang). Die Patienten wurden vor jeder Injektion mit Hilfe einer speziell angefertigten Einverständniserklärung (siehe Abbildung 33 und 34 im Anhang) über Wirkung, Risiken und Nebenwirkungen einer Behandlung mit Botulinumtoxin A aufgeklärt.

Bei allen drei Gruppen waren die möglichen Injektionspunkte, die abhängig vom klinischen Bild injiziert wurden, im Augenbereich, im perioralen Areal und im Platysmabereich praktisch identisch. Diese variierten jedoch individuell zwischen den Injektionsbehandlungen während der Therapie. Injiziert wurde nasal und temporal im Ober- und Unterlidbereich sowie an der temporalen Orbitakante (Musculus orbicularis oculi, Pars orbitalis und palpebralis). Diese Bereiche entsprechen den Punkten 1 bis 6 und 9 bis 10 (siehe Abbildung 13).

Zur Minimierung von Nebenwirkungen wurden bestimmte Injektionsregionen vermieden. Damit das Toxin nicht in den Musculus levator palpebrae oder Musculus rectus superior diffundiert und dadurch eine Ptosis oder eine vertikale Motilitätsstörung mit Diplopie verursacht wird, wurde der mittlere Teil des Oberlids bei der Injektion ausgespart. Im nasalen Unterlidbereich wurde nicht zu weit medial injiziert, um die aktive Tränenpumpe nicht zu beeinflussen und die Häufigkeit einer Epiphora zu senken. Hier wurde auch darauf geachtet, sehr oberflächlich die Dosis zu applizieren. In diesem Bereich befindet sich der Musculus obliquus inferior sehr nahe an der äußeren Haut, so dass vertikal versetzte und schräg stehende Doppelbilder auftreten könnten. Bei Bedarf wurde zusätzlich im Bereich der Stirn (M. corrugator supercilii und M. procerus) oberhalb der Augenbrauen (präseptal) eingespritzt. Es sind die Punkte 7 und 8 in der Abbildung 13.

Im perioralen Bereich (M. orbicularis oris, M. mentalis, M. depressor labii inferioris) durfte nicht zu weit lateral appliziert werden, um diese Region nicht zu lähmen und den Sprech- und 
Schluckvorgang nicht zu gefährden. In der Abbildung 13 entspricht dieses den Punkten 11 bis 14. In der Abbildung 13 sind die Punkte A und B gesondert aufgezeichnet. Diese Injektionsstellen entsprechen dem Musculus zygomaticus major et minor. Je nach Stärke und Ausprägung der Symptomatik wurde auch dort appliziert, um die Verkrampfungen im Mundwinkelbereich zu entlasten.

In Abhängigkeit des individuellen, klinischen und subjektiven Beschwerdebildes wurde auch im Platysmabereich behandelt. Die Punkte 15 bis 20 verdeutlichen diesen Bereich in der Abbildung 13.

Die Injektionen erfolgten subkutan, um die tiefer liegende Muskulatur und die damit verbundenen mögliche unerwünschte Mitreaktion zu vermeiden. Tabelle 2 veranschaulicht welche Rolle die Muskeln bei der menschlichen Mimik und in der Integration des Gesichtsausdruckes spielen. Des Weiteren geht es um die möglichen Injektionsorte zur Therapie der beschriebenen fazialen Dyskinesien. 


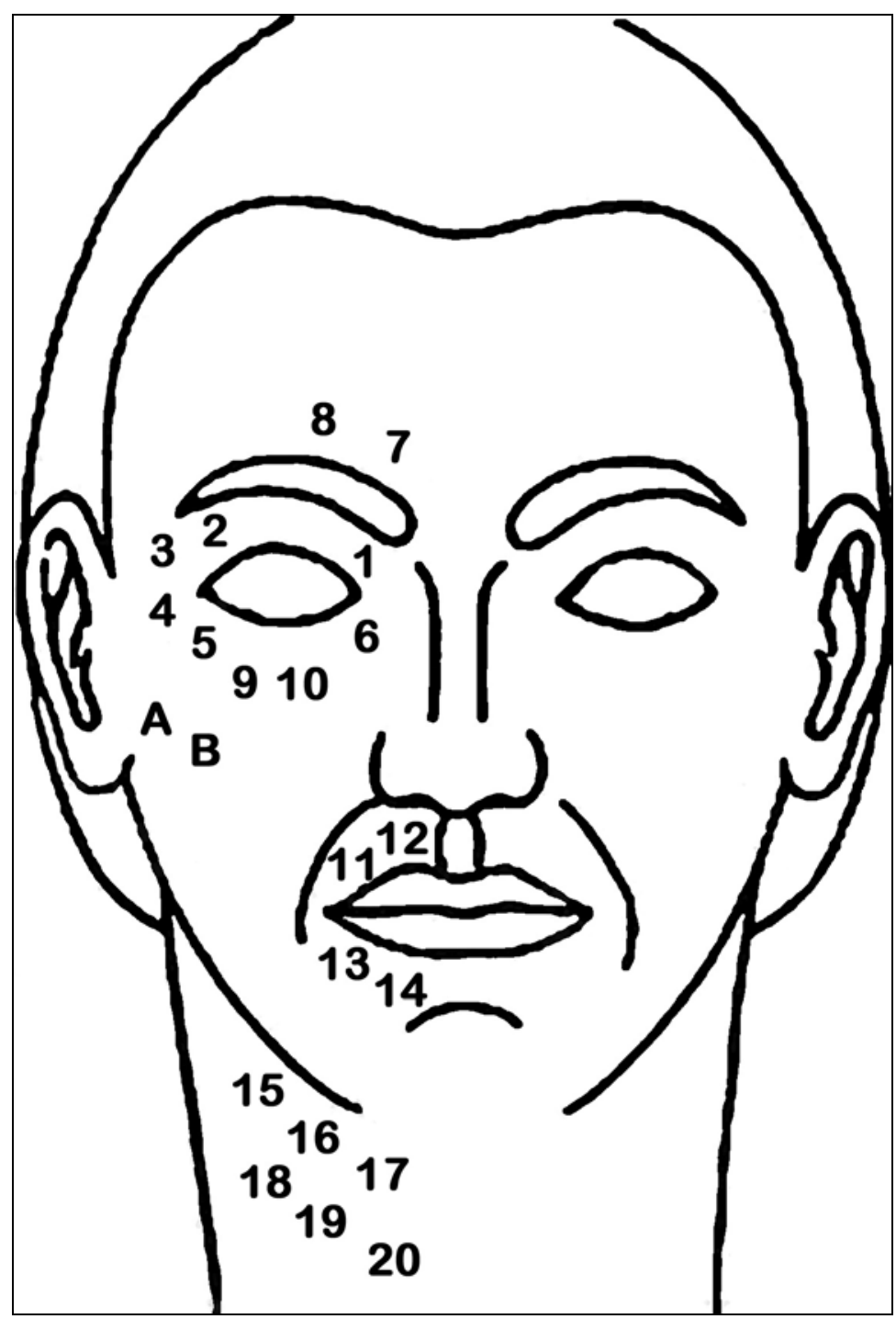

Abbildung 13: Schematische Darstellung der Injektionsorte

Die Punkte 1-6, 9 und 10 stellen den Bereich des M. orbicularis oculi (pars orbitalis und palpebralis) dar.

Die Punkte 7 und 8 entsprechen dem Stirnbereich (M. frontalis), dem M. procerus und M. corrugator. Die Buchstaben A und B kennzeichnen den $\mathrm{M}$. zygomaticus major et minor. Dem perioralen Bereich, M. orbicularis oris, mentalis, depressor labii inferioris, wurden die Zahlen 11-14 zugeordnet. Der Hautmuskel des Halses, das Platysma wurde mit den Zahlen 15-20 beziffert. 


\begin{tabular}{|c|c|c|}
\hline $\begin{array}{c}\text { Injektionsorte und } \\
\text { dazugehörige Muskeln }\end{array}$ & Innervation & Funktion \\
\hline \multicolumn{3}{|l|}{ Punkte 1-6; 9, 10} \\
\hline $\begin{array}{l}\text { M.orbicularis oculi } \\
\text { A: Pars orbitalis } \\
\text { B: Pars palpebralis }\end{array}$ & N. facialis & $\begin{array}{l}\text { Lidschluss, Brauensenker } \\
\text { Lidschlagreflex, Blinzeln }\end{array}$ \\
\hline \multicolumn{3}{|l|}{ Punkte 7 und 8} \\
\hline $\begin{array}{l}\text { M. corrugator supercilii } \\
\text { M. procerus }\end{array}$ & N. facialis & $\begin{array}{l}\text { Haut der Augenbraue wird nach } \\
\text { medial unten gezogen } \\
\text { Bei Kontraktion Querfalte über } \\
\text { der Nasenwurzel }\end{array}$ \\
\hline \multicolumn{3}{|l|}{ Punkte A und B } \\
\hline $\begin{array}{l}\text { M. zygomaticus major } \\
\text { M. zygomaticus minor }\end{array}$ & N. facialis & $\begin{array}{c}\text { Hebt den Mundwinkel nach oben } \\
\text { und lateral, Lachen } \\
\text { Vertieft die Nasolabialfalte }\end{array}$ \\
\hline \multicolumn{3}{|l|}{ Punkte 11, 12, 13} \\
\hline $\begin{array}{l}\text { M. levator anguli oris } \\
\text { M. levator labii } \\
\text { superioris } \\
\text { M. orbicularis oris }\end{array}$ & N. facialis & $\begin{array}{c}\text { Hebt den Mundwinkel } \\
\text { Hebt die Oberlippe } \\
\text { Lippenschluss, Sprechen, Essen, } \\
\text { Trinken }\end{array}$ \\
\hline \multicolumn{3}{|l|}{ Punkte 14} \\
\hline M. mentalis & N. facialis & Erzeugt die Kinn-Lippen-Furche \\
\hline \multicolumn{3}{|l|}{ Punkte 15-20 } \\
\hline Platysma & N. facialis & $\begin{array}{l}\text { Zieht den Unterkiefer und den } \\
\text { Mundwinkel herab }\end{array}$ \\
\hline
\end{tabular}

Tabelle 2: Injektionsorte mit dazugehörigen Muskeln und deren Funktionen (modifiziert nach Grablowitz 2004, Seite 118) 


\subsection{Fragestellung}

Bis zum heutigen Zeitpunkt gibt es zahlreiche Untersuchungen zum Einsatz von Botulinumtoxin bei verschiedenen Krankheitsbildern. Untersucht wurden ebenfalls die von uns ausgewerteten Erkrankungen Blepharospasmus, Spasmus hemifacialis und Synkinesien. Die hohe Besserungspotenz der Substanz bei den hier relevanten Erkrankungen ist seit langem bekannt, auch im Patientengut der HNO-Klinik der Universitätsmedizin Göttingen. Es gibt jedoch nur wenige Langzeitbeobachtungsanalysen und häufig sind in den Betrachtungen verschiedene Erkrankungen zusammengefasst (Mejia et al. 2005, Mauriello und Aljian 1991, Mauriello et al. 1996a,b, Nüßgens und Roggenkämper 1995, Snir et al. 2003, Wang und Jankovic 1998, Hsiung et al. 2002).

Gegenstand dieser Analyse war die Langzeitbetrachtung der Injektionsdynamik der Botulinumtoxin-Therapie beim Blepharospasmus, beim Spasmus hemifacialis und bei Synkinesien nach Fazialisdefektheilung im Hinblick auf

1. die verabreichte Gesamtdosis

2. die Dosisentwicklung an verschiedenen Injektionspunkten sowie

3. Topik-Änderungen von verschiedenen Injektionspunkten in der mimischen Muskulatur.

Hierfür wurden Patienten ausgewählt, die konstant von einer Verbesserung nach der Injektionstherapie berichteten. 


\section{Material und Methoden}

\subsection{Patientengut}

Für die Auswertung der Analyse wurden insgesamt 80 Patienten mit fazialen Hyperkinesien aus der Datenbank der Botulinumtoxin-Spezialsprechstunde der Hals-Nasen-Ohrenklinik der Universitätsmedizin Göttingen nach den von uns gewählten Kriterien im untersuchten Zeitraum ausgewählt. Voraussetzung für die Aufnahme in die Auswertung war die Anzahl der Injektionen, unabhängig von der Zeitdauer zwischen den Injektionen, denn diese waren sehr variabel und individuell. Als Mindestanzahl wurden 10 Behandlungen angesetzt. Für diese Analyse wurden ausschließlich Patienten ausgewählt, die auf eine Therapie mit Botulinumtoxin A angesprochen haben. Es handelt sich um eine retrospektive Analyse der ausgewählten Daten.

Dabei waren die Erkrankungen wie folgt verteilt: Blepharospasmus 30 Patienten, Spasmus facialis 31 Patienten und Synkinesien nach Fazialisdefektheilung 19 Patienten. Es handelte sich um 59 weibliche und 21 männliche Patienten. 25 Frauen und 5 Männer befanden sich in der Blepharospasmus-Gruppe. Die Spasmus-facialis-Gruppe schloss 19 Frauen und 12 Männer ein. In der Behandlungsgruppe der Synkinesien waren es 16 Frauen und 3 Männer (siehe Abbildung 14).

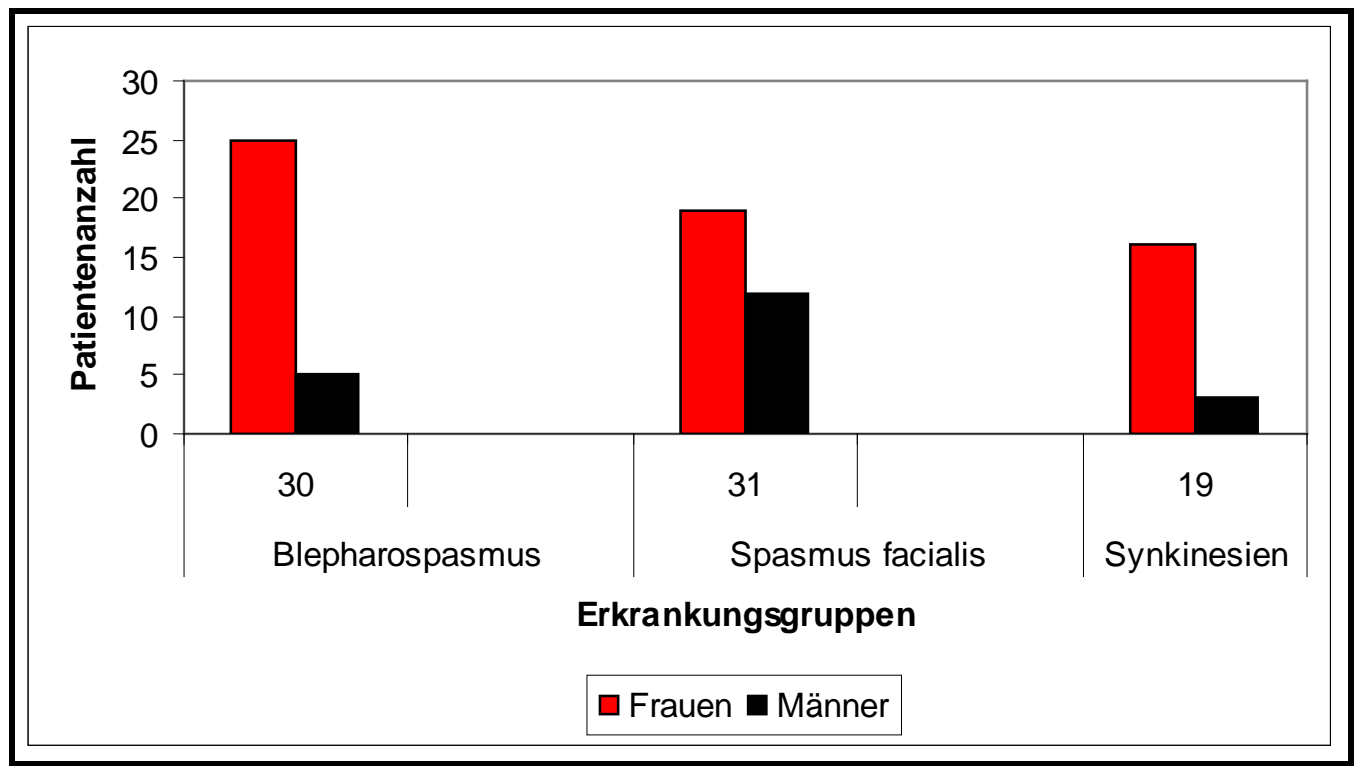

Abbildung 14: Patientenanzahl und Geschlechtsverteilung in den Behandlungsgruppen. 
Die durchschnittliche Behandlungsdauer betrug 8,5 Jahre mit durchschnittlich 23 Behandlungen (zwischen 10 und maximal 40 Injektionsbehandlungen). Insgesamt wurden 1862 Injektionen durchgeführt.

Für die Erhebung der Daten haben wir Patienten, die sich aufgrund eines Blepharospasmus, Spasmus facialis und Synkinesien nach Fazialisdefektheilung vorstellten, in die retrospektive Analyse eingeschlossen. Die Behandlungsgruppen wurden gemeinsam und getrennt voneinander betrachtet.

In der Blepharospasmus-Gruppe befanden sich insgesamt 30 Patienten. 3 Patienten davon litten an einem Meige-Syndrom, einer davon zusätzlich an einem Bruxismus. Bei einem weiteren Patienten lag eine mitochondriale Myopathie vor. Bei allen Patienten zeigte sich die Beschwerdesymptomatik beidseits. Bei einigen Patienten bestand jedoch eine seitendifferente Ausprägung der Beschwerden.

In der Gruppe der Patienten mit Spasmus facialis waren insgesamt 31 Patienten. Bei allen Patienten wurde zur Ursachenabklärung bzw. zum Ausschluss einer Raumforderung oder einer Gefäßanomalie eine Magnetresonanztomographie (MRT) des Schädels (häufig extern) durchgeführt. Bei 26 Patienten bestand ein idiopathischer Spasmus facialis ohne eruierbare Ursache mit unauffälligem MRT-Befund. Eine Gefäßanomalie im Kleinhirnbrückenwinkel wurde bei 3 Patienten diagnostiziert. Alle Patienten wurden über die chirurgischen Möglichkeiten (mikrovaskuläre Dekompressionsoperation) aufgeklärt, hatten sich jedoch zugunsten der Botulinumtoxin-Therapie entschieden. Bei jeweils einem Patienten bestand bereits ein Zustand nach einem neurochirurgischen Eingriff und nach einer StapesplastikOperation (siehe Abbildung 15). 


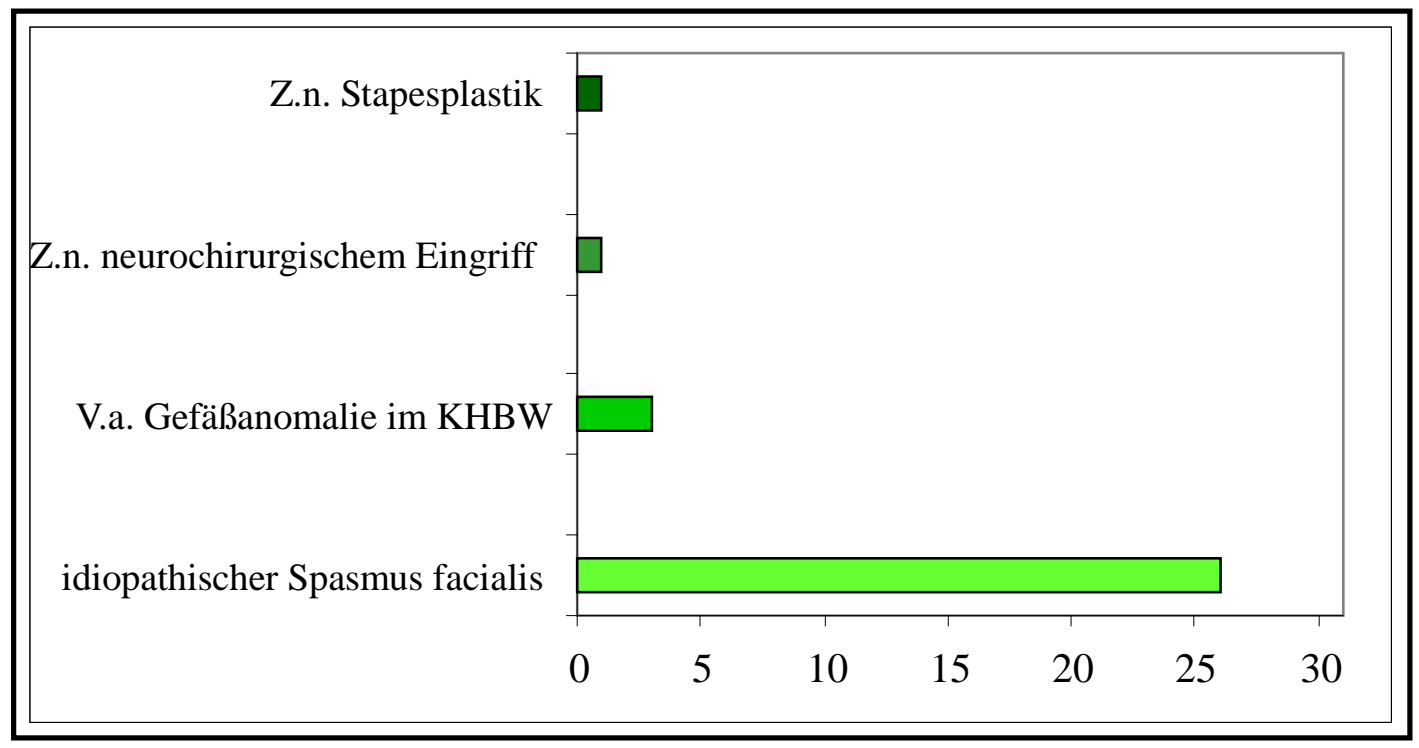

Abbildung 15: Diagnose der Patienten mit Spasmus facialis.

(KHBW= Kleinhirnbrückenwinkel)

19 Patienten befanden sich in der Synkinesien-Gruppe. Bei 2 Patienten erfolgte eine Exstirpation eines Akustikusneurinoms mit darauf folgender peripheren Fazialisparese (einmal rechts, einmal links). 7 Patienten erlitten eine idiopathische Fazialisparese (sog. Bell`sche Parese), in 4 Fällen rechts-, in 3 Fällen linksseitig lokalisiert. Aufgrund eines Zoster oticus bestand bei 4 Patienten eine periphere Fazialisparese, zweimal rechts und links. Bei 6 Patienten bestand ein Zustand nach Akustikusneurinom-Operation mit danach erfolgter Hypoglossus-Fazialis-Anastomose, in 5 Fällen rechts und in einem Fall linksseitig lokalisiert, bei denen es jedoch immer zu einer Fazialisdefektheilung kam (siehe Abbildung 16). 


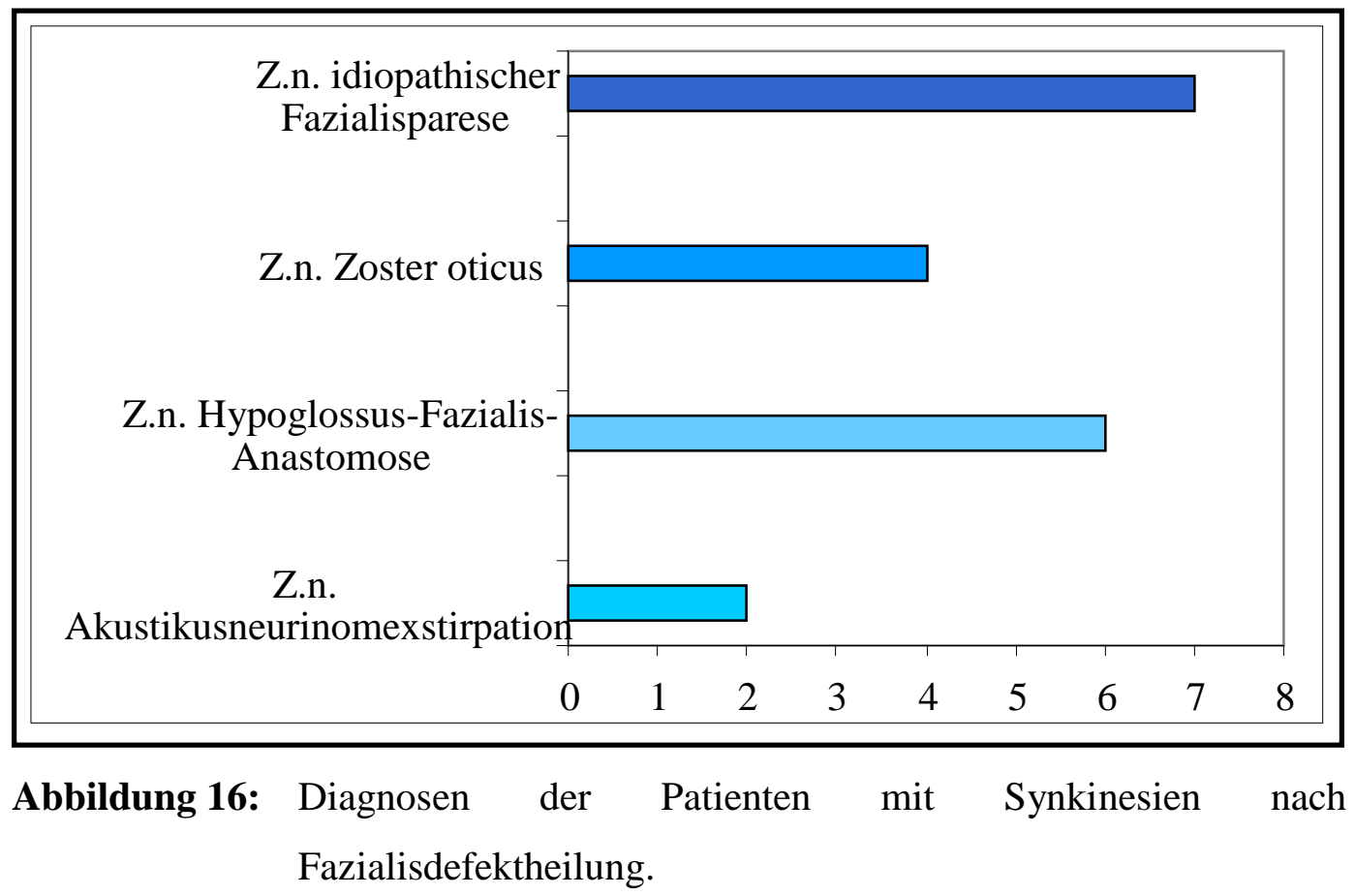

Aus der (aus den Akten entnommenen) Anamnese geht hervor, dass sich die Patienten in allen drei Gruppen durch das Beschwerdebild im täglichen Leben sehr beeinträchtigt, sogar stigmatisiert gefühlt haben. Da bei vielen der Patienten eine medikamentöse Therapie keinen Erfolg gezeigt hatte und sogar mit zum Teil erheblichen Nebenwirkungen einherging, stellten sich diese Patienten zur Therapie mit Botulinumtoxin vor.

\subsection{Datenerhebung}

Es wurde bei jedem Patienten die Gesamtdosis pro Behandlung ermittelt und ein Mittelwert aus allen Patienten bei jeder Injektion errechnet. Es wurden dabei alle injizierten Regionen (periokulär, perioral und Platysmabereich) in die Berechnung der durchschnittlichen Dosis miteinbezogen. Diese Ergebnisse wurden graphisch dargestellt. Um unterscheiden zu können, ob unsere Ergebnisse mit der Anzahl der Behandlung zusammenhängen, wurden die Patienten in entsprechende Gruppen eingeteilt.

Es wurden Gruppen gebildet, die jeweils 10, 20, 30 und 40 Behandlungen erhielten. Die Gruppe mit 10 Behandlungen beinhaltet alle Patienten aus der Blepharospasmus-, Spasmus facialis- und Synkinesien-Gruppe ( $n=80)$. Die Patientengruppe mit 20 Behandlungen umfasst insgesamt 41 Patienten. 30 Behandlungen bekamen 25 Patienten. Die letzte Gruppe mit der 
umfangreichsten Therapieanzahl (40 Injektionen) schließt 4, 2 und 3 Patienten ein. In dieser Einteilung sind Patienten eingeschlossen, die zum entsprechenden Behandlungszeitraum einen Wert haben.

Ferner wurde das Patientengut in Erkrankungsgruppen eingeteilt, um eine Aussage treffen zu können, ob die Gesamtdosis von dem Faktor Erkrankungsbild abhängig ist.

Bei jedem Patienten wurde pro Injektion auch die Anzahl der Injektionspunkte analysiert. Es wurden bei den Injektionen die periokuläre Region, periorale Region und die Platysmaregion mitberücksichtigt. Anschließend wurde bei jeder Injektionsbehandlung ein Mittelwert der Injektionspunkte gebildet. Es konnte auch aufgeschlüsselt werden, welche Punkte zu welchem Zeitpunkt eine Veränderung erfahren haben.

Des Weiteren wurde bei jedem Patienten eine Einzeldosis pro Injektionspunkt und bei jeder Injektion bestimmt. Daraus ließ sich ein Mittelwert pro Punkt und Injektion berechnen. Auch hier wurden die periokuläre, periorale und Platysmaregion mitberücksichtigt. Das wurde graphisch dargestellt und ausgewertet.

Außerdem wurde die interessante Frage nach dem Nebenwirkungsprofil analysiert.

Es konnte prozentual dargestellt werden, wie häufig eine Ptosis, ein hängender Mundwinkel, ein Lagophthalmus, eine Sicca-Symptomatik, die Epiphora oder ein Hämatom aufgetreten sind.

\subsection{Statistische Methodik}

In der deskriptiven Analyse wurden abhängig vom Endpunkt Häufigkeiten und Prozente oder Mittelwerte und Standardabweichungen angegeben. Zur graphischen Veranschaulichung wurden Histogramme, Boxplots und Mittelwertabbildungen angefertigt. Boxplots zeigen den Median und den Interquartilabstand. Ausreißer sind speziell gekennzeichnet. Mittelwertabbildungen zeigen den Mittelwert mit einem Streumaß (Standardabweichung oder Konfidenzintervall).

Um die relevanten Endpunkte hinsichtlich eines Gruppen- (Erkrankungsgruppe), Zeiteffektes und deren Wechselwirkung überprüfen zu können, wurden statistische Tests durchgeführt. Hierfür wurde eine zweifaktorielle ANOVA für Messwiederholungen berechnet. Das Signifikanzniveau wird auf $\alpha=5 \%$ festgelegt. Das bedeutet, dass ein $\mathrm{p}$-Wert kleiner als 0.05 auf eine statistische Signifikanz hinweist. Für die weiterführenden Paarvergleiche wurde eine 
Bonferroni-Adjustierung durchgeführt. Für die Paarvergleiche wurde ein abhängiger T-Test verwendet. 


\section{Ergebnisse}

\subsection{Botulinumtoxin-Therapie bei fazialen Hyperkinesien}

Die durchgeführte retrospektive Analyse schloss 80 Patienten aus der Botulinumtoxin-Spezialsprechstunde der Hals-Nasen-Ohrenklinik der Universitätsmedizin Göttingen ein. Es handelte sich um 59 weibliche und 21 männliche Patienten. Im Mittel waren die Patienten 68 Jahre alt. Die Spannweite des Alters lag zwischen 41 und 93 Jahren. Das ermittelte Alter wurde vom Geburtsdatum an bis zu der letzten Behandlung errechnet.

In der Blepharospasmus-Gruppe war die älteste Patientin 90 und die jüngste 46 Jahre alt. Im Mittel betrug das Alter 72 Jahre. Jeweils 4 Patienten befanden sich im Alter zwischen 40-59 und 60-69 Jahren. Bei 14 Patienten betrug das Alter zwischen 70-79 und bei 8 Patienten zwischen 80-90 Jahren (siehe Abbildung 17).

Die Gruppe der Patienten mit einem Spasmus facialis war im Durchschnitt 67,5 Jahre alt, die älteste Patientin war 93 und die jüngste 25 Jahre alt. Zwischen 40-59 Lebensjahr befanden sich in dieser Gruppe 8 Patienten. Bei 20 Patienten betrug das Alter zwischen 60-69 Jahren. Jeweils 7 bzw. 6 Patienten befanden sich im Alter zwischen 70-79 und 80-93 Jahren (siehe Abbildung 17).

Das Durchschnittsalter der Patienten in der Synkinesien-Gruppe erreichte 62 Jahre. Die älteste Patientin war 82 und die jüngste 25 Jahre. Das Alter bei jeweils einem Patienten betrug 25 und 82 Jahre. Bei 5 und 7 Patienten ist das Alter zwischen 40-59 und 60-69 Jahren zu verzeichnen. Zwischen 70-79 Jahren befanden sich 7 Patienten in dieser Gruppe (siehe Abbildung 17). 


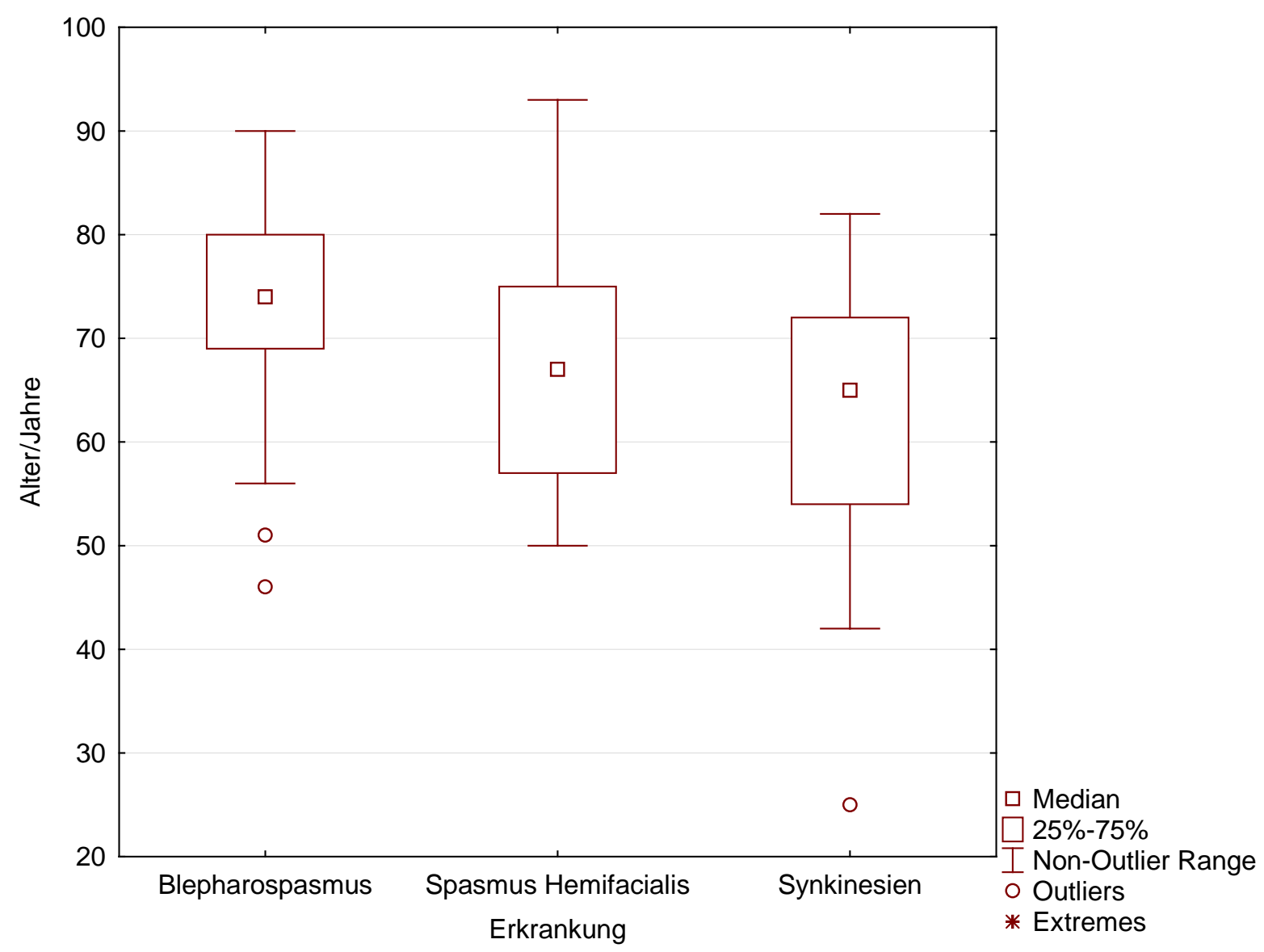

Abbildung 17: Verteilung des Alters der Patienten in den Erkrankungsgruppen:

Blepharospasmus-Gruppe $(n=30)$,

Spasmus-facialis-Gruppe $(\mathrm{n}=31)$,

Synkinesien-Gruppe ( $\mathrm{n}=19)$

Die Behandlungsdauer betrug bei allen 80 Patienten durchschnittlich 8,5 Jahre. In dieser Zeit wurden im Mittel 23 Behandlungen durchgeführt.

In der Blepharospasmus-Gruppe betrug die Behandlungsdauer durchschnittlich 8 Jahre, wobei die Erkrankten im Minimum 3 und im Maximum 15 Jahren therapiert wurden. Es wurden insgesamt 690 Injektionen durchgeführt.

Die Spasmus-facialis-Gruppe wurde im Durchschnitt 8,8 Jahre mit minimal 3 und maximal 15 Jahren behandelt. Die Anzahl der durchgeführten Injektionen betrug 692. 
In der Gruppe der Synkinesien betrug die durchschnittliche Behandlungsdauer 9 Jahre, im Minimum 3 und maximal 17 Jahre. Insgesamt 480 Injektionen wurden über die Zeit appliziert (siehe Tabelle 3).

\begin{tabular}{|c|c|}
\hline & Behandlungsdauer in Jahren \\
\hline Erkrankungen & $\begin{array}{c}\text { Durchschnittliche } \\
\text { Behandlungsdauer (Min-Max) }\end{array}$ \\
\hline Blepharospasmus & $8(3-15)$ \\
\hline Spasmus facialis & 8,8 (3-15) \\
\hline Synkinesien & $9(3-17)$ \\
\hline
\end{tabular}

Tabelle 3: Behandlungsdauer der Erkrankungsgruppen

\subsection{Therapiehäufigkeit}

Jeder Patient bekam mindestens 10 Behandlungen mit Botulinumtoxin. Die maximale Behandlungsanzahl umfasste 40 Injektionen. Diese Maximalbehandlung erhielten insgesamt 9 Patienten aus den 3 oben beschriebenen Gruppen. Die zeitlichen Abstände zwischen den Injektionen waren intra- und interindividuell sehr unterschiedlich. Der Grund für die zeitlich schwankenden Injektionsabstände liegt primär darin begründet, dass die erneute Injektion sich nicht nach einem festen Schema richtet, sondern nach der individuellen klinischen Symptomatik des einzelnen Patienten.

\subsubsection{Therapiehäufigkeit bei Blepharospasmus, Spasmus facialis und Synkinesien}

Bei Patienten mit Blepharospasmus betrugen die zeitlichen Abstände zwischen den Injektionen im Mittel 19 Wochen (4,5 Monate). Patienten mit Spasmus facialis kamen im Durchschnitt alle 23 Wochen (5,5 Monate) zur erneuten Injektion. In der Gruppe der Patienten mit Synkinesien waren es 21 Wochen (5 Monate) bis zur notwendigen Folgeinjektion (siehe Tabelle 4). 


\begin{tabular}{|c|c|}
\hline Erkrankung & $\begin{array}{c}\text { Erforderliche Re-Injektionen } \\
\text { (in Wochen) }\end{array}$ \\
\hline Blepharospasmus & 19 \\
\hline Spasmus facialis & 23 \\
\hline Synkinesien & 21 \\
\hline
\end{tabular}

Tabelle 4: Zeitintervall bis zur notwendigen Wiederbehandlung.

Bei allen untersuchten Fällen mit Blepharospasmus lag eine beidseitige Symptomatik vor. Einige Patienten gaben Unterschiede in der Ausprägung der Beschwerden an, welche entweder von Anfang an oder im Laufe der Behandlung auftraten. Daher kam es in einigen Fällen im Verlauf zum Einsatz von seitendifferenten Botox ${ }^{\circledR}-D o s e n$ an einzelnen Injektionsorten. In der zweiten Gruppe (Spasmus facialis) befanden sich 17 Fälle mit rechtsseitigem und 14 Fälle mit linksseitigem Spasmus facialis. Die Ausprägung der fazialen Hyperkinesien war zu Behandlungsbeginn und im Behandlungsverlauf nicht bei allen Patienten identisch. Aus diesem Grund kam es auch hier zu Dosisänderungen am Injektionsort und zur Hinzunahme neuer Injektionspunkte im Laufe der Behandlungszeit.

Im Falle der Synkinesien bestand bei 12 Patienten eine rechtsseitige und bei 7 Patienten eine linksseitige Symptomatik. Auch hier wurden Änderungen der Dosis und der Injektionsorte im Laufe der Behandlung beobachtet.

\subsection{Entwicklung der durchschnittlichen Gesamtdosis über die Behandlungsjahre}

Zunächst wurde die Gesamtdosis über die Behandlungsjahre erfasst und ein Mittelwert aller Patienten pro Injektion errechnet. In diesem Patientengut befanden sich alle Patienten unabhängig von der Behandlungsanzahl. Bis zu der 10. Injektion befanden sich alle 80 Patienten in der Behandlung. Bei der 20. und 30. Injektion waren es 41 und 25 Patienten. Insgesamt neun Patienten bekamen 40 Injektionen. Es konnte mit einer graphischen Darstellung gezeigt werden, dass die eingesetzte Dosis mit den Behandlungsjahren zunimmt (siehe Abbildung 
18). Es wurde im Durchschnitt (Standardabweichung) mit 24 Einheiten (13) bei der ersten Behandlung begonnen. Bei der 10. Behandlung waren es durchschnittlich 29,8 Einheiten (15). Die Dosis stieg dann bei der 20. Behandlung auf 35,2 Einheiten (17,1) über 37,9 Einheiten $(14,8)$ bei der 30. Injektion an. Durchschnittlich 42,8 Einheiten $(13,9)$ wurden bei der 40. Injektion appliziert. Die Abbildung 18 zeigt jedoch, dass die Werte einer großen Varianz unterliegen.

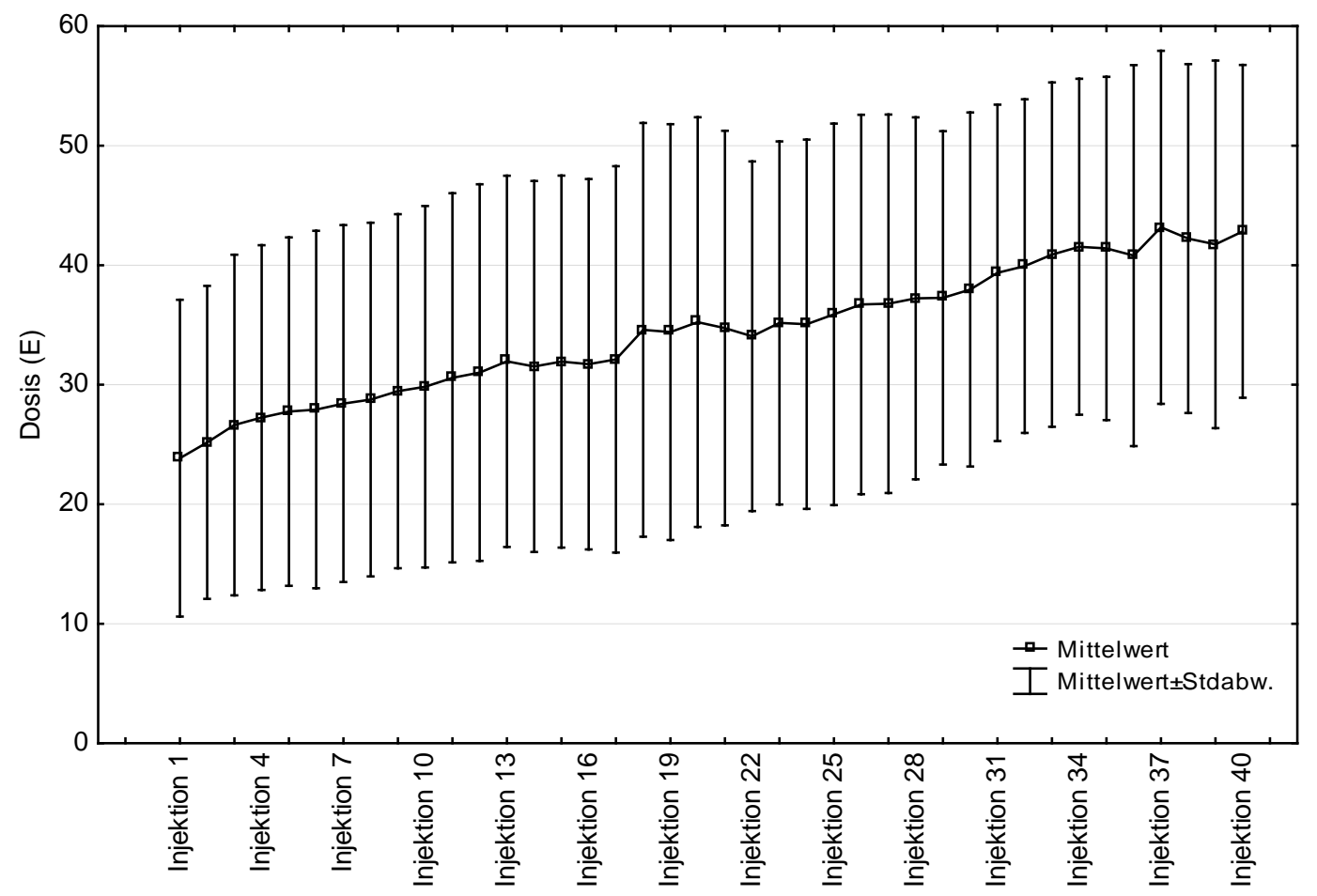

Abbildung 18: Mittlere Botulinumtoxin-Dosis mit Standardabweichung (E) bei allen Patienten pro Injektion ( $n=80)$.

Wie bereits im Abschnitt Material und Methoden beschrieben, wurden im Folgenden die Krankheitsgruppen getrennt betrachtet. Es zeigte sich aber auch in jeder Erkrankungsgruppe, dass eine Dosiserhöhung über die Jahre zu verzeichnen war (siehe Abbildung 19-21).

Die höchste durchschnittliche Anfangsdosis (Standardabweichung) bestand bei Patienten mit Blepharospasmus, diese betrug 33,3 Einheiten $(13,8)$ bei der ersten Behandlung. Die Dosis stieg auf 42,4 $(13,9)$ und 49,5 Einheiten $(15,8)$ bei der 10. und 20. Injektion an. Eine weitere, geringere Erhöhung der durchschnittlichen Dosis konnte bei der 30. und 40. Behandlung mit 50,6 (10,3) und 53,4 Einheiten $(10,6)$ beobachtet werden. Alle Patienten erhielten 10 
Injektionen. 20 Injektionen erhielten sechzehn Patienten, 30 Injektionen neun Patienten. Bis zur 40. Injektion konnten nur vier Patienten aus der Blepharospasmus-Gruppe ausgewertet werden. Auch in dieser Abbildung sind große Varianzen an den Behandlungszeitpunkten zu erkennen (siehe Abb. 19).

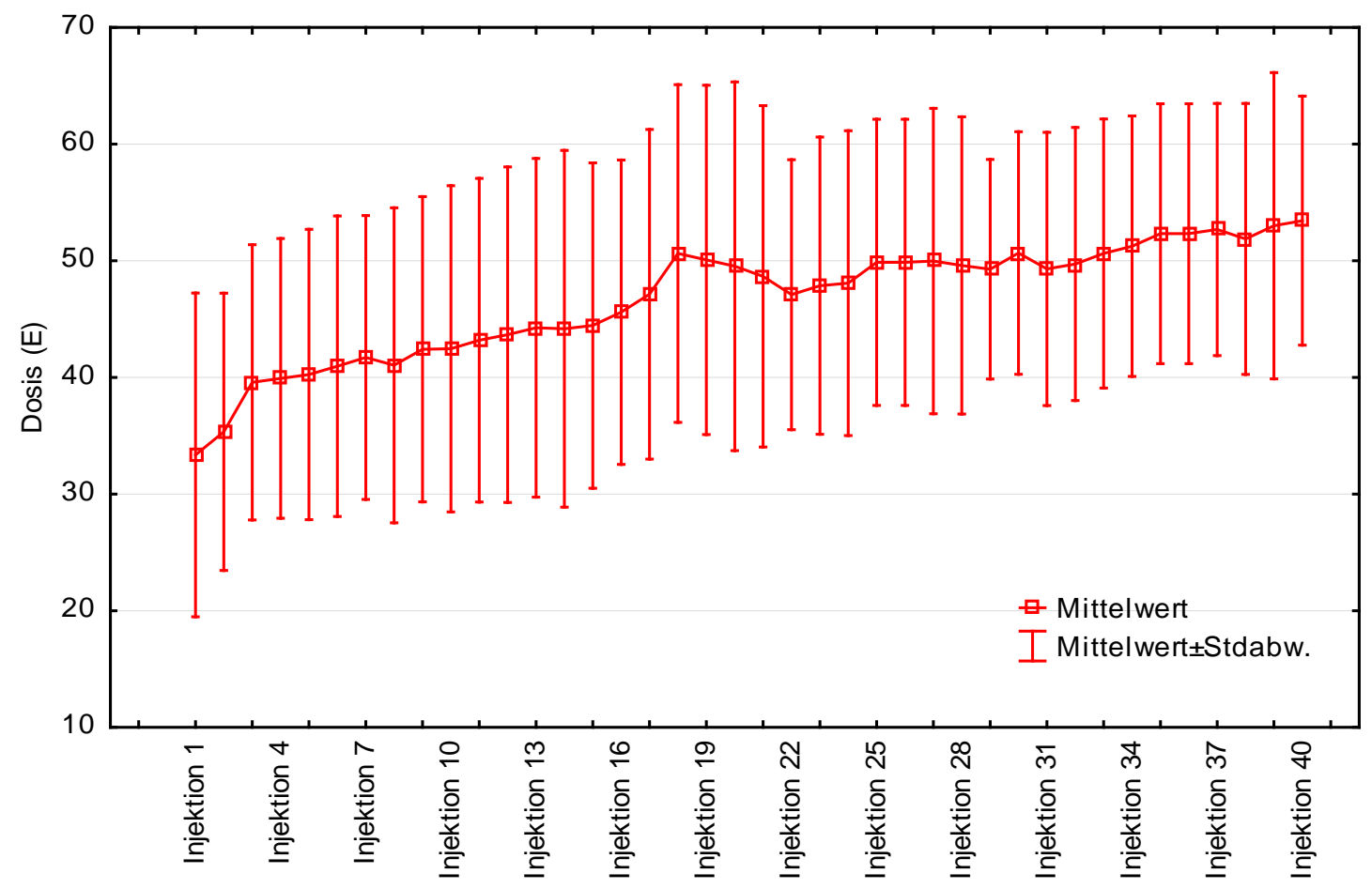

Abbildung 19: Mittlere Dosis mit Standardabweichung (E) in der Blepharospasmusgruppe

( $n=30$ bei 10 Injektionen; $n=16$ bei 20 Injektionen; $n=9$ bei 30 Injektionen; $\mathrm{n}=4$ bei 40 Injektionen).

Die Gruppe der Patienten mit einem Spasmus facialis begann im Durchschnitt (Standardabweichung) mit 20,5 Einheiten $(9,2)$ bei der ersten Injektion und stieg auf 24,8 (9,9), 30 (11) und 33,6 (14,1) Einheiten bei der 10., 20. und 30. Behandlung an. Die Dosis lag bei der 40. Injektion im Durchschnitt bei 43,4 (4,7) Einheiten. Auch in dieser Gruppe erhielten alle Patienten 10 Injektionen ( $n=30)$. 20 Behandlungen bekamen fünfzehn Patienten, bei 30 Injektionen waren es neun Patienten. Bis zu der 40. Behandlung konnten in dieser Gruppe nur zwei Patienten ausgewertet werden. Diese Gruppe zeigt ebenso eine deutliche Varianz der Dosiswerte (siehe Abb. 20). 


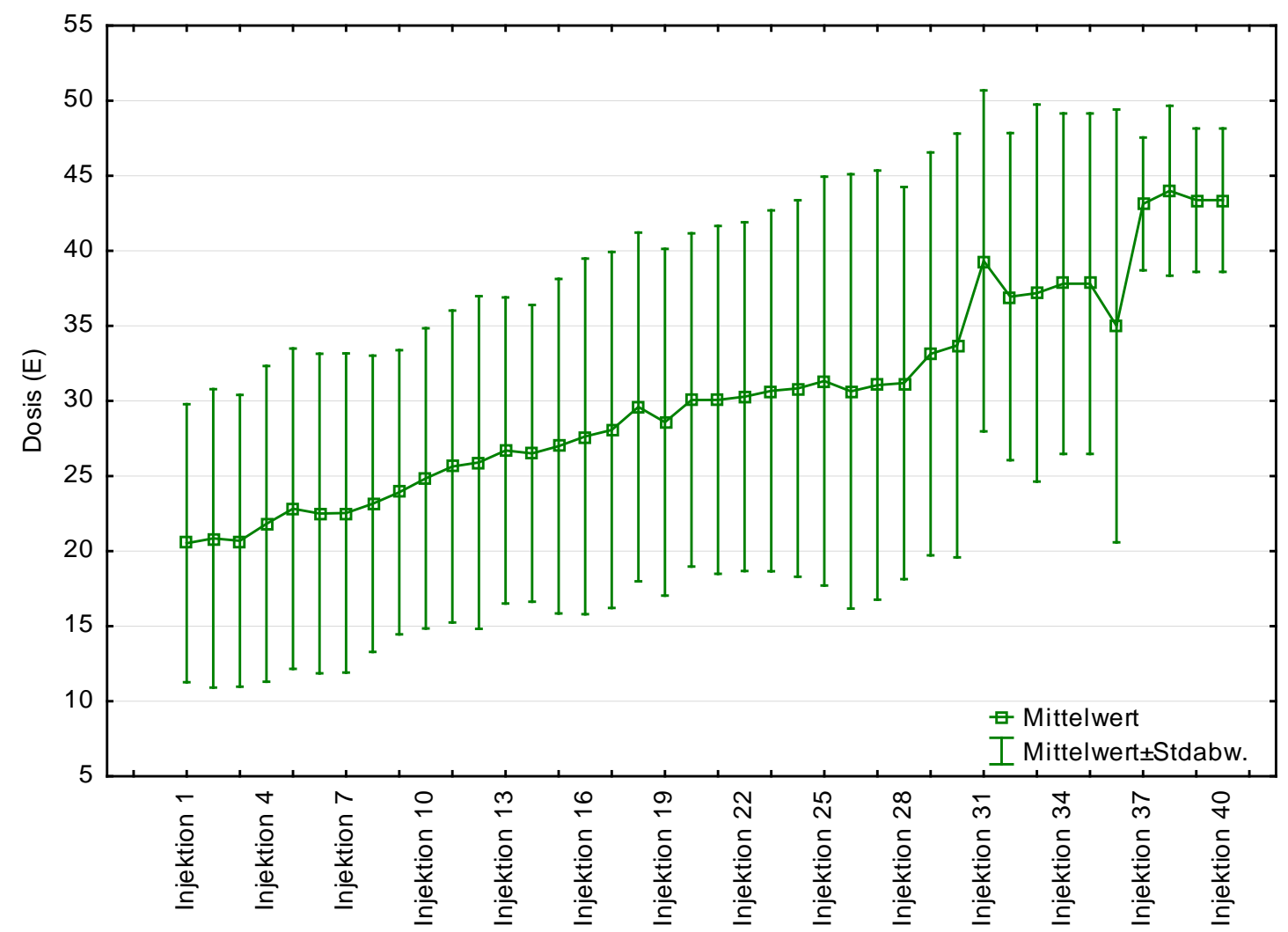

Abbildung 20: Mittlere Dosis mit Standardabweichung (E) in der Spasmusfacialis-Gruppe ( $n=31$ bei 10 Injektionen, $n=15$ bei 20 Injektionen, $n=9$ bei 30 Injektionen, $\mathrm{n}=2$ bei 40 Injektionen).

Die niedrigste durchschnittliche Gesamtdosis erhielt die Patienten-Gruppe mit Synkinesien nach Fazialisdefektheilung. Die Anfangsdosis (Standardabweichung) lag bei $14,3(7,1)$ Einheiten und wurde auf 18 (8,2), 20,1 (6) und 27,1 (7,8) Einheiten bei der 10., 20. und 30. Behandlung erhöht. Zum Zeitpunkt der 40. Behandlung waren es 28,3 (7,2) Einheiten. Es konnten jedoch insgesamt drei Patienten ausgewertet werden. Alle Patienten bekamen 10 Injektionen ( $\mathrm{n}=19)$. Zehn Patienten erhielten 20 und sieben Patienten erhielten 30 Behandlungen. Eine große Varianz der Dosiswerte kann auch hier beobachtet werden (siehe Abb. 21). 


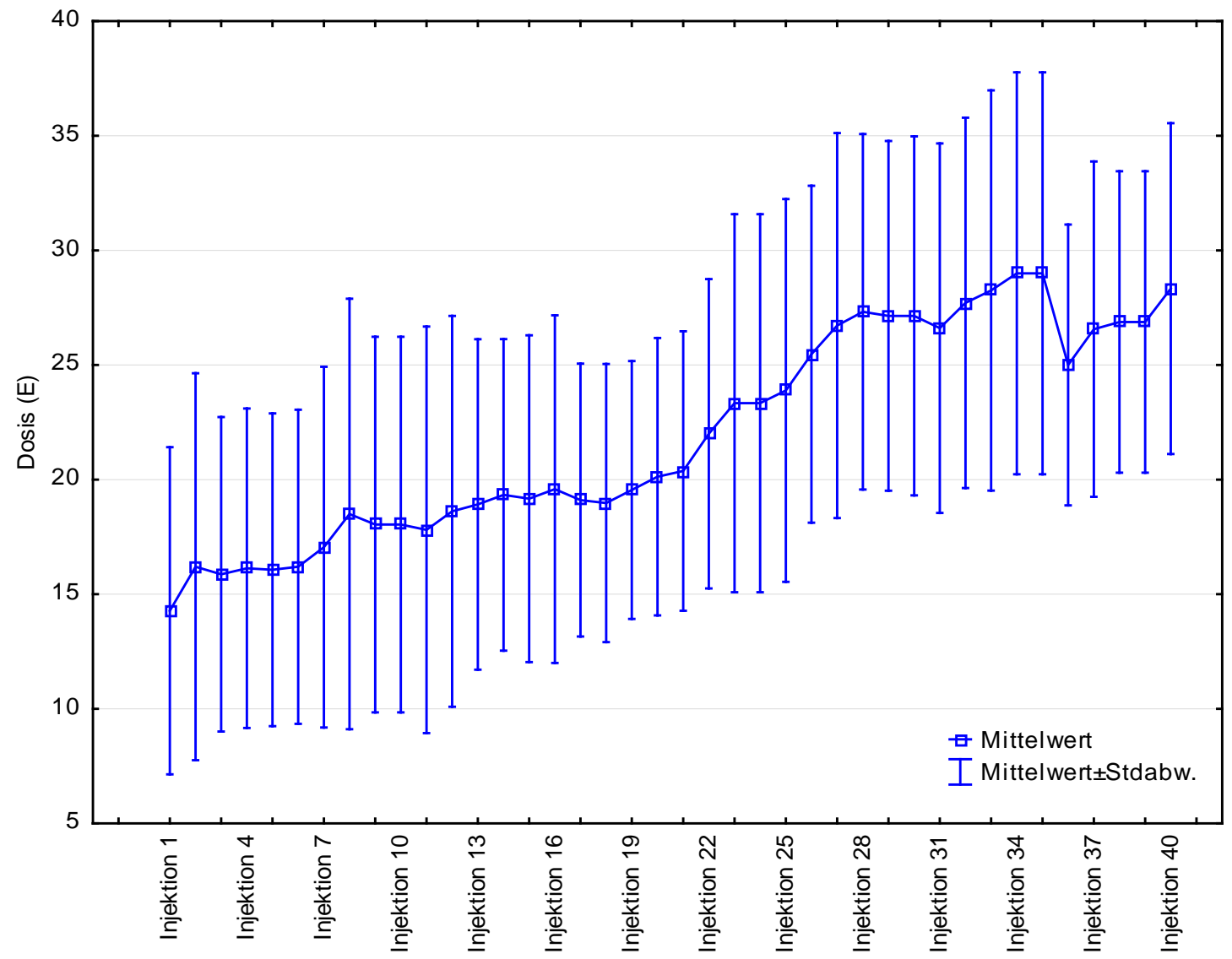

Abbildung 21: Mittlere Dosis und Standardabweichung (E) in der SynkinesienGruppe

( $n=19$ bei 10 Injektinen, $n=10$ bei 20 Injektionen, $n=7$ bei 30 Injektionen, $\mathrm{n}=3$ bei 40 Injektionen).

In der Abbildung 18 konnte eine Steigerung der mittleren Dosis über alle Behandlungsjahre bei allen Patienten gezeigt werden. Die Patientenanzahl ist jedoch zu den angegebenen Zeitpunkten unterschiedlich groß.

In den folgenden statistischen Tests wurden die relevanten Endpunkte hinsichtlich eines Gruppen- (Erkrankungsgruppe), Injektionseffektes und deren Wechselwirkung überprüft. Für den Injektionseffekt wurden jeweils der erste Injektionszeitpunkt mit dem zehnten, zwanzigsten, dreißigsten und vierzigsten Injektionszeitpunkt verglichen. Zu jedem angegebenen Zeitpunkt wurden Patienten eingeschlossen, die zu beiden Zeitpunkten einen entsprechenden Wert hatten. Bei der ersten und 10. Behandlung waren es alle 80 Patienten. Die Patientengruppe mit 20 Behandlungen umfasst 16 Patienten mit Blepharospasmus, 15 
Patienten mit Spasmus facialis und 10 mit Synkinesien $(n=41)$. 30 Behandlungen erhielten 9 Patienten mit Blepharospasmus, 9 mit einem Spasmus facialis und 7 Patienten mit Synkinesien $(n=25) .40$ Behandlungen bekamen 9 Patienten, jeweils 4 BlepharospasmusPatienten, 2 Spasmus facialis-Patienten und 3 Synkinesien-Patienten.

Den Abbildungen 22 bis 25 ist zu entnehmen, dass die Dosis im Mittel bei allen Erkrankungen zum entsprechenden Zeitpunkt ansteigt. Es zeigte sich somit keine signifikante Wechselwirkung zwischen den Gruppen bei allen statistischen Auswertungen. Der p-Wert zeigt keine statistische Signifikanz an (siehe Abb. 22-25, Tabelle 5-8).

Da keine Wechselwirkung gezeigt werden konnte, ist eine Auftrennung der Daten nach Erkrankungsgruppen nicht zwangsläufig nötig.

Ein mittlerer Dosisunterschied konnte von Injektion 1 zu 10 gezeigt werden, da der p-Wert für den Injektionseffekt eine statistische Signifikanz anzeigt (siehe Tabelle 5). In dieser Gruppe befanden sich alle Patienten 80 Patienten.

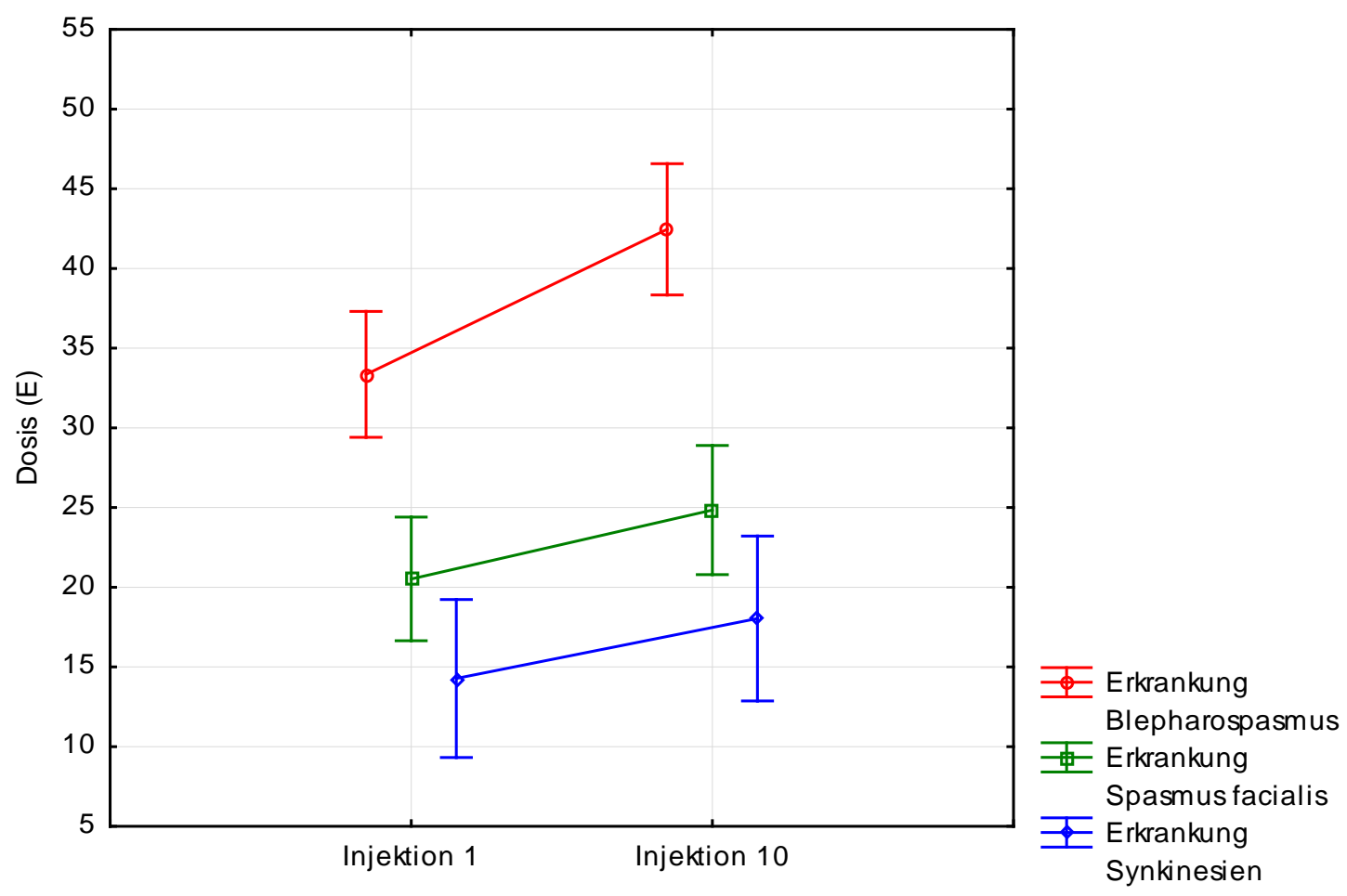

Abbildung 22: Mittlere Dosis (E) mit Konfidenzintervall zum ersten und zehnten Injektionszeitpunkt bei den Erkrankungsgruppen Blepharospasmus $(n=30)$, Spasmus facialis $(n=31)$, Synkinesien $(n=19)$. 


\begin{tabular}{|c|c|c|}
\hline Faktor & p-Wert & Interpretation \\
\hline Erkrankungsgruppe & $\leq 0,00$ & signifikant \\
\hline Injektionseffekt & $\leq 0.01$ & signifikant \\
\hline Wechselwirkung & $\leq 0,11$ & nicht signifikant \\
\hline
\end{tabular}

Tabelle 5: Ergebnisse in Form des p-Wertes der ANOVA mit Messwiederholungen, wobei die Injektionszeitpunkte 1 und 10 betrachtet werden.

Da die Erkrankungsgruppenunterschiede detaillierter beschrieben werden sollen, wurden zu den verschiedenen Injektionszeitpunkten Paarvergleiche durchgeführt (siehe Tabelle 6, 8, 10, 12).

In den Paarvergleichen konnte zu bestimmten Injektionszeitpunkten ein Erkrankungseffekt beobachtet werden. Der Vergleich zwischen den Erkrankungsgruppen Blepharospasmus und Spasmus facialis sowie Blepharospasmus und Synkinesien zeigt zum ersten und zehnten Injektionszeitpunkt einen signifikanten Mittelwertunterschied. Der p-Wert beträgt bei den Injektionszeitpunkten $\leq 0,01$ und zeigt somit eine statistische Signifikanz an (siehe Tabelle 6). Der p-Wert zum ersten und zehnten Injektionszeitpunkt deutet im Paarvergleich der Spasmus facialis- und Synkinesien-Gruppe auf keinen Mittelwertunterschied hin. Somit ist die mittlere Dosis im Laufe der Behandlungsjahre hier vergleichbar (siehe Tabelle 6). 


\begin{tabular}{|c|c|c|c|c|}
\hline & Erkrankungsgruppe & $\begin{array}{l}\text { Mittelwert (SD) } \\
\text { in Einheiten }\end{array}$ & $\begin{array}{c}\text { p-Wert } \\
\text { (adjustiert) }\end{array}$ & Interpretation \\
\hline \multirow{6}{*}{ Injektion 1} & Blepharospasmus & 33,3 (14) & \multirow{2}{*}{$\leq \mathbf{0 , 0 1}$} & \multirow{2}{*}{ signifikant } \\
\hline & Spasmus facialis & $20,5(9,2)$ & & \\
\hline & Blepharospasmus & 33,3 (14) & \multirow{2}{*}{$\leq \mathbf{0 , 0 1}$} & \multirow{2}{*}{ signifikant } \\
\hline & Synkinesien & $14,3(7,1)$ & & \\
\hline & Spasmus facialis & $20,5(9,2)$ & \multirow{2}{*}{$\leq 0,06$} & \multirow{2}{*}{$\begin{array}{c}\text { nicht } \\
\text { signifikant }\end{array}$} \\
\hline & Synkinesien & $14,3(7,1)$ & & \\
\hline \multirow{6}{*}{ Injektion 10} & Blepharospasmus & $42,4(13,9)$ & \multirow{2}{*}{$\leq \mathbf{0 , 0 1}$} & \multirow{2}{*}{ signifikant } \\
\hline & Spasmus facialis & $24,8(9,9)$ & & \\
\hline & Blepharospasmus & $42,4(13,9)$ & \multirow{2}{*}{$\leq \mathbf{0 , 0 1}$} & \multirow{2}{*}{ signifikant } \\
\hline & Synkinesien & $18(8,2)$ & & \\
\hline & Spasmus facialis & $24,8(9,9)$ & \multirow{2}{*}{$\leq 0,06$} & \multirow{2}{*}{$\begin{array}{c}\text { nicht } \\
\text { signifikant }\end{array}$} \\
\hline & Synkinesien & $18(8,2)$ & & \\
\hline
\end{tabular}

Tabelle 6: Paarvergleiche der Erkrankungsgruppen aufgetrennt nach den zwei Injektionszeitpunkten.

Auch zum 20. Injektionszeitpunkt konnte gezeigt werden, dass es zu einem mittleren Dosisunterschied von Injektion 1 zu Injektion 20 gekommen ist (siehe Abb. 23). Hier muss mitberücksichtigt werden, dass die Anzahl der Patienten abgenommen hat, da nicht alle Patienten aus unserer Datenbank 20 Behandlungen bekommen haben ( $n=41 ; 16$ Patienten mit Blepharospasmus, 15 Patienten mit Spasmus facialis und 10 mit Synkinesien). Der p-Wert für den Injektionseffekt zeigt eine statistische Signifikanz an (siehe Tabelle 7).

Im Paarvergleich zwischen Spasmus facialis- und Synkinesie-Gruppe konnte auch hier gezeigt werden, dass die mittlere Dosis über die Jahre vergleichbar ist. Der Mittelwertunterschied ist statistisch nicht signifikant. Zwischen der Blepharospasmus- und Spasmus facialis-Gruppe, Blepharospasmus- und Synkinesien-Gruppe zeigt der p-Wert eine statistische Signifikanz an (siehe Tabelle 8). 


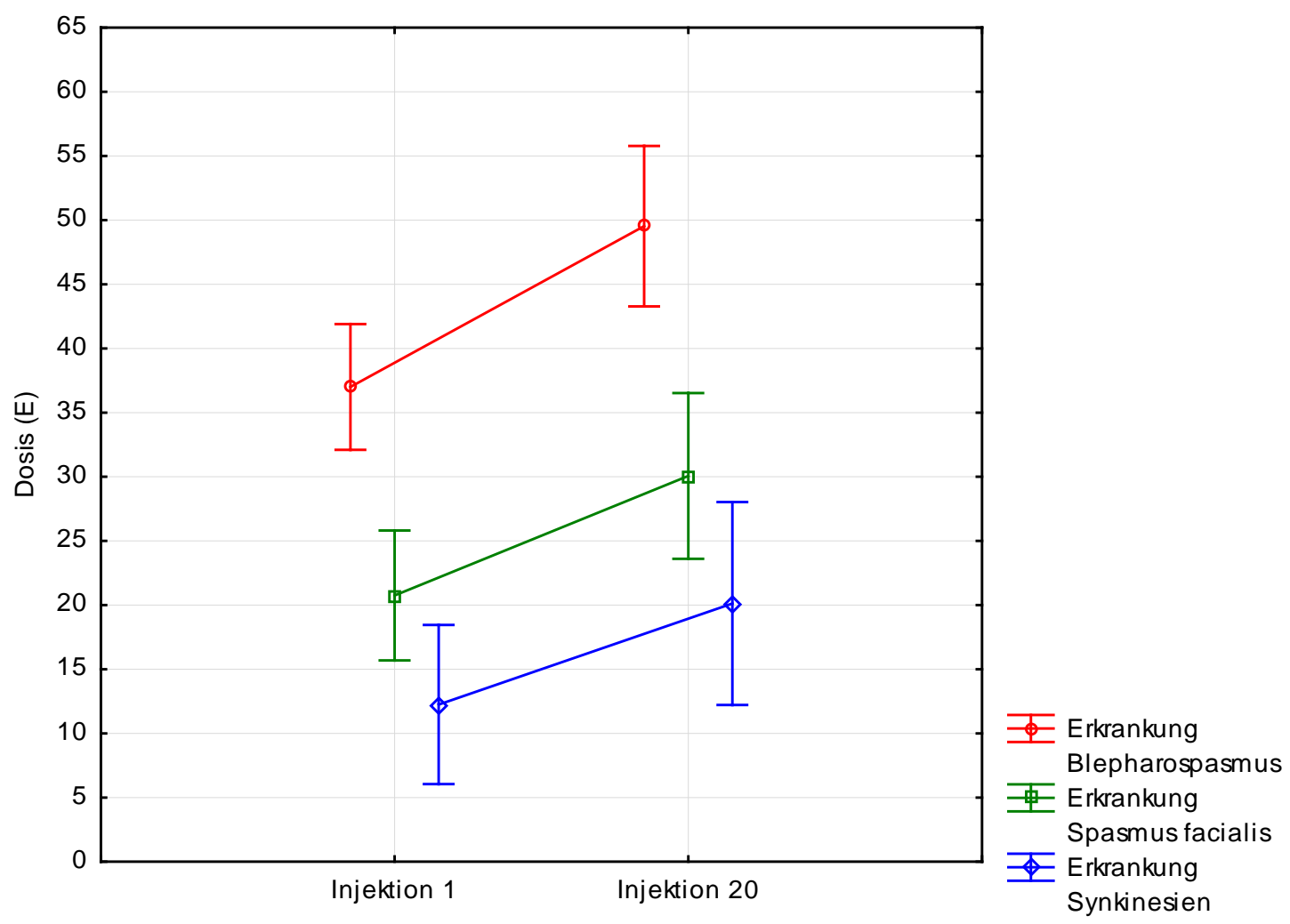

Abbildung 23: Mittlere Dosis (E) mit Konfidenzintervall zum ersten und zwanzigsten Injektionszeitpunkt bei den Erkrankungsgruppen Blepharospasmus $(n=16)$, Spasmus facialis $(n=15)$, Synkinesien $(n=10)$.

\begin{tabular}{|c|c|c|}
\hline Faktor & p-Wert & Interpretation \\
\hline Erkrankungsgruppe & $\leq 0,01$ & signifikant \\
\hline Injektionseffekt & $\leq 0,01$ & signifikant \\
\hline Wechselwirkung & $\leq 0,66$ & nicht signifikant \\
\hline
\end{tabular}

Tabelle 7: Ergebnisse in Form des p-Wertes der ANOVA mit Messwiederholungen, wobei die Injektionszeitpunkte 1 und 20 betrachtet werden. 


\begin{tabular}{|c|c|c|c|c|}
\hline & Erkrankungsgruppe & $\begin{array}{c}\text { Mittelwert (SD) } \\
\text { in Einheiten }\end{array}$ & $\begin{array}{c}\text { p-Wert } \\
\text { (adjustiert) }\end{array}$ & Interpretation \\
\hline & Blepharospasmus & $49,5(15,8)$ & \multirow{2}{*}{$\leq \mathbf{0 , 0 1}$} & signifikant \\
\cline { 2 - 3 } & Spasmus facialis & $30(11)$ & \multirow{2}{*}{$\leq \mathbf{0 , 0 1}$} & signifikant \\
\cline { 2 - 3 } Injektion 20 & Blepharospasmus & $49,5(15,8)$ & $20,1(6)$ & nicht \\
\cline { 2 - 3 } & Synkinesien & $30(11)$ & \multirow{2}{*}{$\leq 0,06$} & signifikant \\
\cline { 2 - 3 } & Spasmus facialis & $20,1(6)$ & &
\end{tabular}

Tabelle 8: Paarvergleiche der Erkrankungsgruppen aufgetrennt nach dem Injektionszeitpunkt.

Zum Zeitpunkt der 30. Injektion wird ein ähnlicher Effekt beobachtet. Auch hier besteht ein Unterschied in der mittleren Dosis zwischen der 1. und der 30. Injektion (siehe p-Wert für Injektionseffekt in Tabelle 9 und Abb. 24). In dieser Gruppe befanden sich nur 25 Patienten (9 Blepharospasmus-Patienten, 9 Spasmus facialis-Patienten und 7 Synkinesien- Patienten) die bis zu diesem Zeitpunkt eine Behandlung bekommen haben.

Im Paarvergleich besteht ein signifikanter Unterschied zwischen der Blepharospasmus- und Synkinesien-Gruppe (siehe p-Wert in Tabelle 10). Im Vergleich zwischen der Blepharospasmus-, Spasmus facialis-Gruppe und Spasmus facialis-, Synkinesien-Gruppe konnte kein statistischer Mittelwertunterschied gezeigt werden (siehe Tabelle 10). 


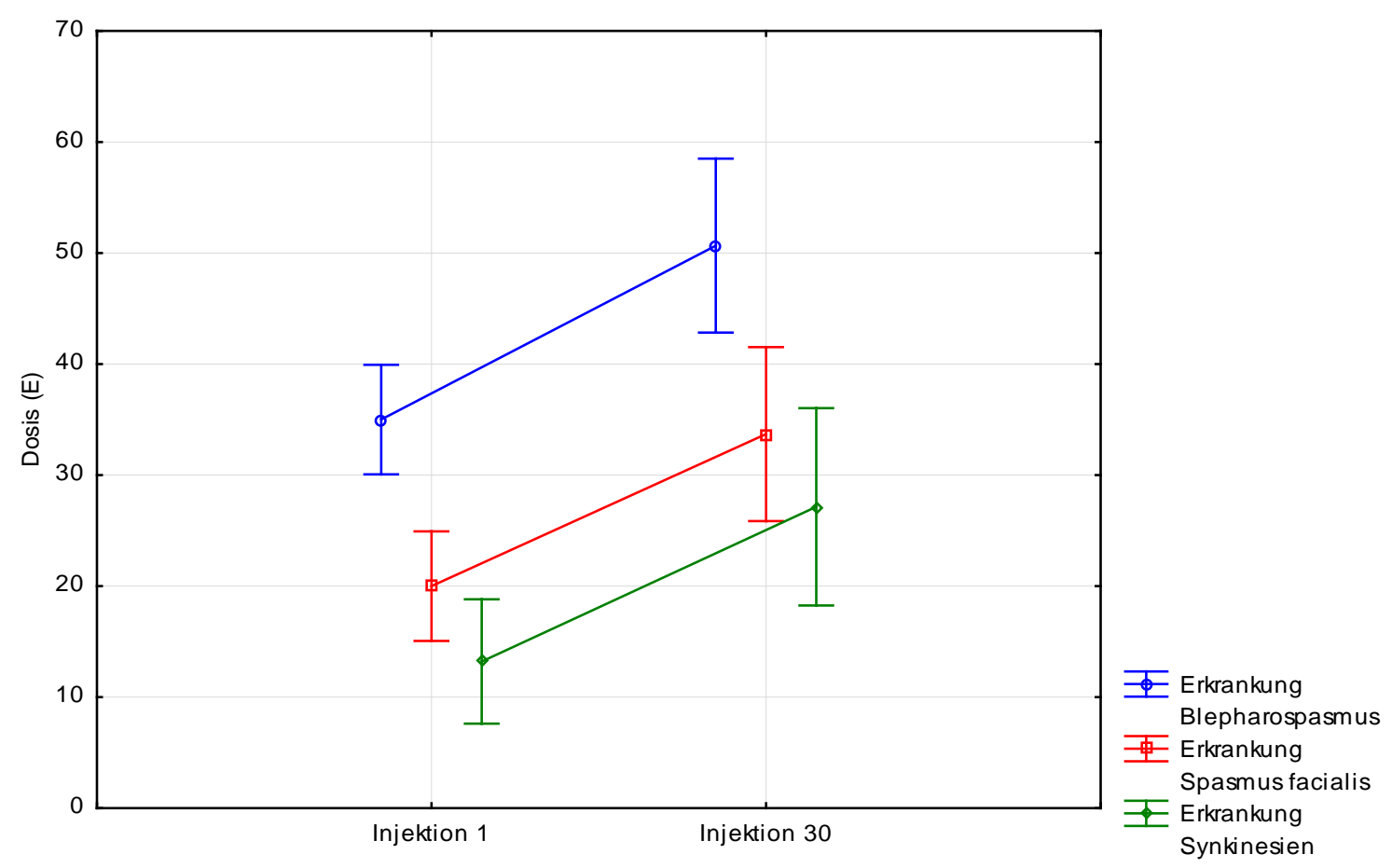

Abbildung 24: Mittlere Dosis (E) mit Konfidenzintervall zum ersten Injektionszeitpunkt und zum dreißigsten Injektionszeitpunkt bei den Erkrankungsgruppen

Blepharospasmus ( $n=9)$, Spasmus facialis $(n=9)$, Synkinesien $(n=7)$.

\begin{tabular}{|c|c|c|}
\hline Faktor & p-Wert & Interpretation \\
\hline Erkrankungsgruppe & $\leq 0,01$ & signifikant \\
\hline Injektionseffekt & $\leq 0,01$ & signifikant \\
\hline Wechselwirkung & $\leq 0,92$ & nicht signifikant \\
\hline
\end{tabular}

Tabelle 9: Ergebnisse in Form des p-Wertes der ANOVA mit Messwiederholungen, wobei die Injektionszeitpunkte 1 und 30 betrachtet werden. 


\begin{tabular}{|c|c|c|c|c|}
\hline & Erkrankungsgruppe & $\begin{array}{l}\text { Mittelwert (SD) } \\
\text { in Einheiten }\end{array}$ & $\begin{array}{c}\text { p-Wert } \\
\text { (adjustiert) }\end{array}$ & $\begin{array}{l}\text { Interpretati } \\
\text { on }\end{array}$ \\
\hline \multirow{6}{*}{ Injektion 30} & Blepharospasmus & $50,5(10,3)$ & \multirow{2}{*}{$\leq 0,06$} & \multirow{2}{*}{$\begin{array}{c}\text { nicht } \\
\text { signifikant }\end{array}$} \\
\hline & Spasmus facialis & $33,6(14,1)$ & & \\
\hline & Blepharospasmus & $50,5(10,3)$ & \multirow{2}{*}{$\leq \mathbf{0 , 0 1}$} & \multirow{2}{*}{ signifikant } \\
\hline & Synkinesien & $27,1(7,8)$ & & \\
\hline & Spasmus facialis & $33,6(14,1)$ & \multirow{2}{*}{$\leq 1$} & \multirow{2}{*}{$\begin{array}{c}\text { nicht } \\
\text { signifikant }\end{array}$} \\
\hline & Synkinesien & $27,1(7,8)$ & & \\
\hline
\end{tabular}

Tabelle 10: Paarvergleiche der Erkrankungsgruppen aufgetrennt nach dem Injektionszeitpunkt.

Zum 40. Injektionszeitpunkt wird auch ein statistisch signifikanter Mittelwertunterschied bei der mittleren Injektionsdosis beobachtet (siehe Tabelle 11, p-Wert für Injektionseffekt). Die Anzahl der behandelten Patienten in der Gruppe beinhaltet 9 Personen (jeweils 4 Blepharospasmus-, 2 Spasmus facialis- und 3 Synkinesien-Patienten).

Nur der Paarvergleich zwischen der Blepharospasmus- und Synkinesie-Gruppe ergab eine statistische Signifikanz für die mittlere Behandlungsdosis über die Jahre (siehe Tabelle 12 und Abb.25). Der p-Wert für den Vergleich zwischen den Blepharospasmus-Patienten, Spasmus facialis-Patienten und Spasmus facialis-Patienten, Synkinesie-Patienten deutet auf keinen statistisch signifikanten Mittelwertunterschied in der Dosis hin. 


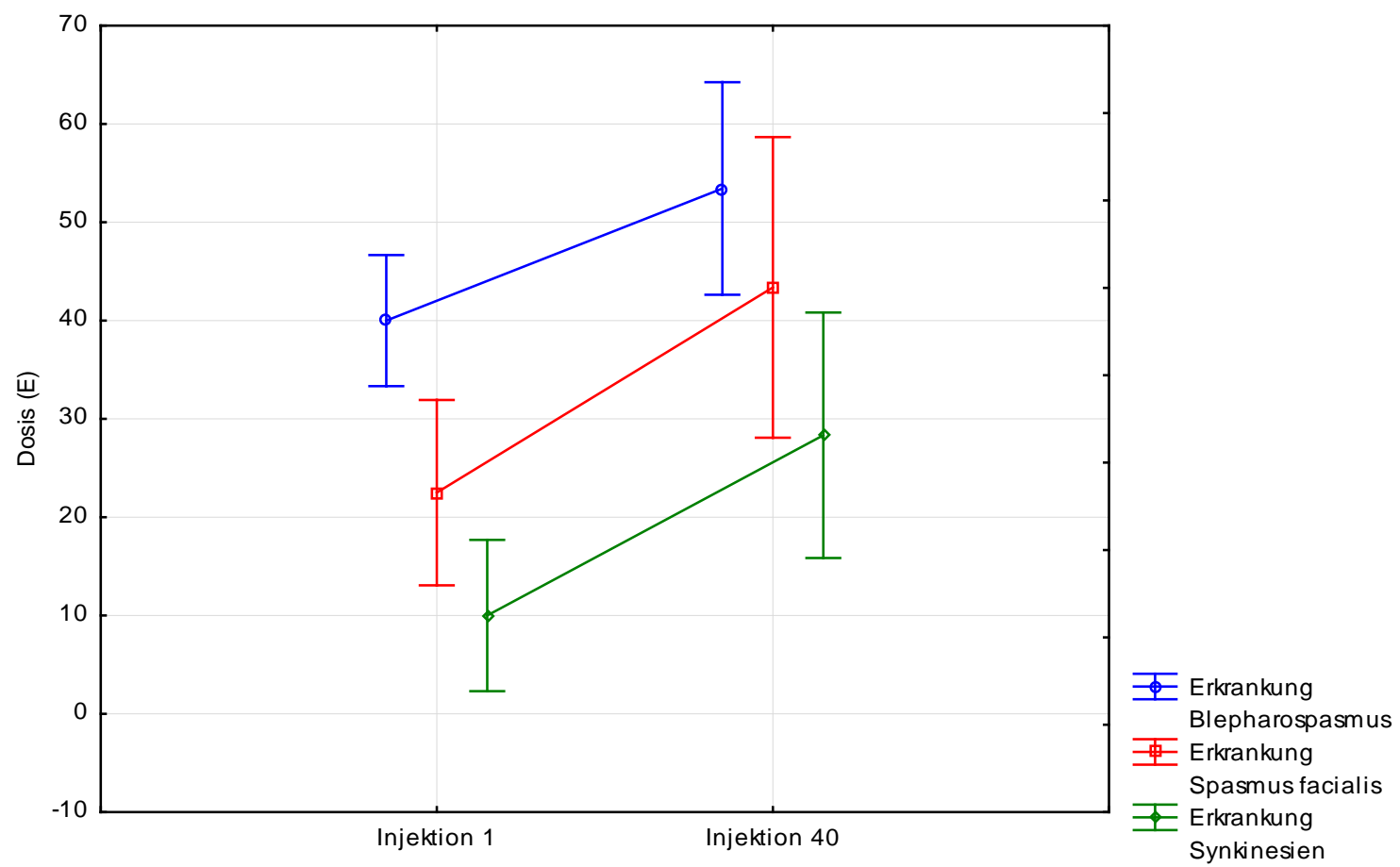

Abbildung 25: Mittlere Dosis (E) mit Konfidenzintervall zum ersten I und vierzigsten Injektionszeitpunkt bei den Erkrankungsgruppen Blepharospasmus ( $n=4)$, Spasmus facialis $(n=2)$, Synkinesien $(n=2)$.

\begin{tabular}{|c|c|c|}
\hline Faktor & p-Wert & Interpretation \\
\hline Erkrankungsgruppe & $\leq 0,01$ & signifikant \\
\hline Injektionseffekt & $\leq 0,01$ & signifikant \\
\hline Wechselwirkung & $\leq 0,45$ & nicht signifikant \\
\hline
\end{tabular}

Tabelle 11: Ergebnisse in Form des p-Wertes der ANOVA mit Messwiederholungen, wobei die Injektionszeitpunkte 1 und 40 betrachtet werden. 


\begin{tabular}{|c|c|c|c|c|}
\hline & Erkrankungsgruppe & $\begin{array}{l}\text { Mittelwert (SD) } \\
\text { in Einheiten }\end{array}$ & $\begin{array}{c}\text { p-Wert } \\
\text { (adjustiert) }\end{array}$ & Interpretation \\
\hline \multirow{6}{*}{ Injektion 40} & Blepharospasmus & $53,4(10,6)$ & \multirow{2}{*}{$\leq 0,29$} & \multirow{2}{*}{$\begin{array}{c}\text { nicht } \\
\text { signifikant }\end{array}$} \\
\hline & Spasmus facialis & $43,4(4,7)$ & & \\
\hline & Blepharospasmus & $53,4(10,6)$ & \multirow{2}{*}{$\leq \mathbf{0 , 0 1}$} & \multirow{2}{*}{ signifikant } \\
\hline & Synkinesien & $28,3(7,2)$ & & \\
\hline & Spasmus facialis & $43,4(4,7)$ & \multirow{2}{*}{$\leq 0,08$} & \multirow{2}{*}{$\begin{array}{c}\text { nicht } \\
\text { signifikant }\end{array}$} \\
\hline & Synkinesien & $28,3(7,2)$ & & \\
\hline
\end{tabular}

Tabelle 12: Paarvergleiche der Erkrankungsgruppen aufgetrennt nach dem Injektionszeitpunkt.

\subsection{Entwicklung der Anzahl der Injektionspunkte in den Behandlungsjahren}

Im Folgenden wurde die Anzahl der Injektionspunkte pro Injektion und Patient ermittelt und anschließend wurde daraus ein Mittelwert gebildet. In dieser Betrachtung konnte nachgewiesen werden, dass die Anzahl der Injektionspunkte in den Gruppen „Spasmus facialis“ und „Synkinesien“ kontinuierlich angestiegen ist. Bei Patienten mit einem Blepharospasmus fehlt dieser Trend (siehe Abbildung 26).

Auch in dieser Betrachtung muss berücksichtigt werden, dass in jeder Patientengruppe zu den verschiedenen Behandlungszeitpunkten eine unterschiedliche Patientenanzahl gegeben ist. 


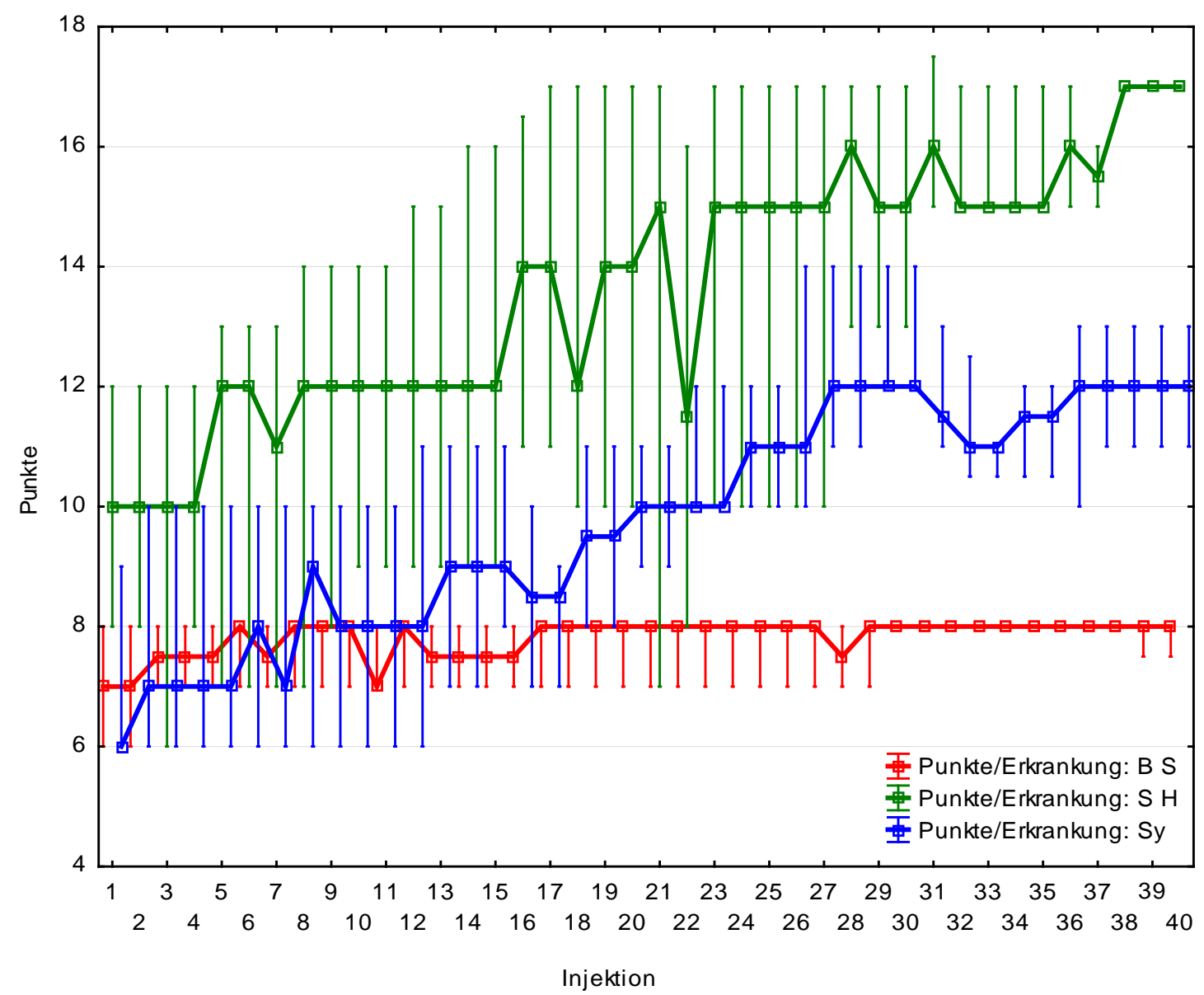

Abbildung 26: Mittlere Injektionspunktanzahl mit Standardabweichung pro Behandlung bei Blepharospasmus ( $\mathrm{n}=30)$, Spasmus facialis $(\mathrm{n}=31)$ und Synkinesien $(\mathrm{n}=19)$.

Man kann bei Blepharospasmus-Patienten erkennen, dass die Punkteanzahl relativ konstant bleibt. Als Standard-Schema wurden die Punkte 1 bis 6 injiziert. Durchschnittlich wurde jedoch mit 7 Injektionsorten begonnen. Im weiteren Verlauf ist die Anzahl der Injektionsorte kaum angestiegen und betrug konstant 8 Injektionspunkte. Es gab auch kaum Unterschiede zwischen dem rechten und dem linken Auge. Bei Patienten, bei denen die Symptomatik sehr ausgeprägt war, wurden, je nach anatomischer Lokalisation des Spasmus, die Punkte 7 und 8 oder 9 und 10 zusätzlich gespritzt (7 und 8 kennzeichnen die Augenbrauenregion; 9 und 10 entsprechen dem unteren Rand des M. orbicularis oculi). Diese Punkte kamen aber auch später im weiteren Therapieverlauf hinzu, wenn der Patient anfänglich nur die Injektion am Auge gewünscht hatte, obwohl die Spasmen auch im Stirnbereich eindeutig zu erkennen wa- 
ren. Dieses Tatsache betrifft sowohl die Spasmus-facialis- als auch die Synkinesien-Gruppe (siehe Abbildung 26, Tabelle 13).

Bei Spasmus facialis wurde die Therapie mit Botulinumtoxin im Mittel mit 10 Injektionspunkten begonnen. Die Anzahl der Injektionspunkte wuchs mit der Therapiedauer von 14 bei der 20. Injektion bis 17 Punkte bei der 40. Behandlung (siehe Abbildung 26, Tabelle 13). Bei 38 \% (12 von 31 Personen) der Patienten mit Spasmus facialis wurde die Behandlung der Hyperkinesien im Augenbereich begonnen. Davon wurden 4 Pat. (33\%) bis zu der letzten Behandlung nur in der periokulären Region injiziert. In 61\% (19 von 31) des Patientengutes begannen wir die Injektion bei der ersten Vorstellung zusätzlich in der Platysmaregion und im perioralen Bereich.

In der dritten Gruppe der Patienten mit Synkinesien wurde durchschnittlich mit 6 Punkten begonnen. Die Anzahl der Punkte stieg auf 10 und 12 Injektionspunkte bei der 20. und 30. Behandlung. Bei der 40. Injektion waren es bereits 12 Injektionspunkte (siehe Abbildung 26, Tabelle 13). Die Behandlung wurde in $58 \%$ im Augenbereich und in $42 \%$ zusätzlich im Platysmabereich und in der perioralen Region durchgeführt. Zu der Periorbitalregion zählen die Standardpunkte 1-6 und die Punkte 7-10 (das entspricht der Augenbrauenregion und dem Unterrand des M. orbicularis oculi), obwohl diese nicht bei jedem Patienten bei der ersten Injektion appliziert wurden. Die Anzahl und Verteilung der Injektionspunkte war intra- und interindividuell different. Diese Verteilung schwankte auch zwischen den Injektionsbehandlungen. Dies beruht darauf, dass man sich bei jeder Injektionsbehandlung nach dem Beschwerdebild des Patienten gerichtet hatte.

\begin{tabular}{|l|c|c|c|c|c|c|c|}
\hline \multirow{2}{*}{ Erkrankung } & \multicolumn{7}{|c|}{ Anzahl der Injektionspunkte pro Behandlung } \\
& $\mathbf{1}$ & $\Delta$ & $\mathbf{2 0}$ & $\Delta$ & $\mathbf{3 0}$ & $\Delta$ & $\mathbf{4 0}$ \\
\hline Blepharospasmus & $\mathbf{7}$ & $\mathbf{1}$ & $\mathbf{8}$ & $\mathbf{0}$ & $\mathbf{8}$ & $\mathbf{0}$ & $\mathbf{8}$ \\
\hline & & & & & & & \\
\hline Spasmus facialis & $\mathbf{1 0}$ & $\mathbf{4}$ & $\mathbf{1 4}$ & $\mathbf{1}$ & $\mathbf{1 5}$ & $\mathbf{2}$ & $\mathbf{1 7}$ \\
\hline & & & & & & & \\
\hline Synkinesien & $\mathbf{6}$ & $\mathbf{4}$ & $\mathbf{1 0}$ & $\mathbf{2}$ & $\mathbf{1 2}$ & $\mathbf{0}$ & 12 \\
\hline
\end{tabular}

Tabelle 13: $\quad$ Anstieg der Injektionspunkte im Laufe der Behandlungsjahre. $\Delta=$ Differenz der Injektionspunkte zwischen den Behandlungen 
Im weiteren Verlauf wird wiederum eine ANOVA, wie oben dargestellt, durchgeführt. Jedoch ist nun der abhängige Endpunkt die Punktezahl. In unserer Analyse wurden somit die drei Erkrankungsgruppen auch bezüglich der Injektionspunkte zu den Zeitpunkten der ersten, der zehnten, der zwanzigsten, der dreißigsten und der vierzigsten Behandlung miteinander verglichen und auf Wechselwirkung, Erkrankungseffekt, Zeiteffekt und deren Signifikanz geprüft. Zu den angegebenen Zeitpunkten wurden Patienten eingeschlossen, die zu beiden Zeitpunkten einen Wert hatten, sodass an den verschiedenen Behandlungen eine unterschiedliche Patientenanzahl vorhanden war.

Da in diesem Fall eine Wechselwirkung vorliegt (siehe p-Wert Tabelle 14, 16, 18, 20), wird nach den verschiedenen Gruppen aufgetrennt, um mögliche Injektionszeiteffekte aufzudecken.

In der Abbildung 27 ist zu erkennen, dass die Anzahl der Punkte (Standardabweichung) in der Spasmus facialis-Gruppe und Synkinesie-Gruppe von der ersten bis zu der 10. Behandlung ansteigt. In der Spasmus facialis-Gruppe wurde mit 9,5 (3,3), in der SynkinesieGruppe mit 7,3 (2,4) Injektionspunkten begonnen. Bei der 10. Injektion waren es 11,3 (3,6) und 8,5 (2,8) Punkte. Der p-Wert zeigt hier also eine statistische Signifikanz an. Bei Blepharospasmus-Patienten bleibt der Anstieg aus. Es wurden zu Beginn und zum Zeitpunkt der 10. Injektion 7-7,4 (1,3-1,2) Punkte verabreicht. In dieser Gruppe konnten alle 80 Patienten betrachtet werden (siehe Tabelle 15). 


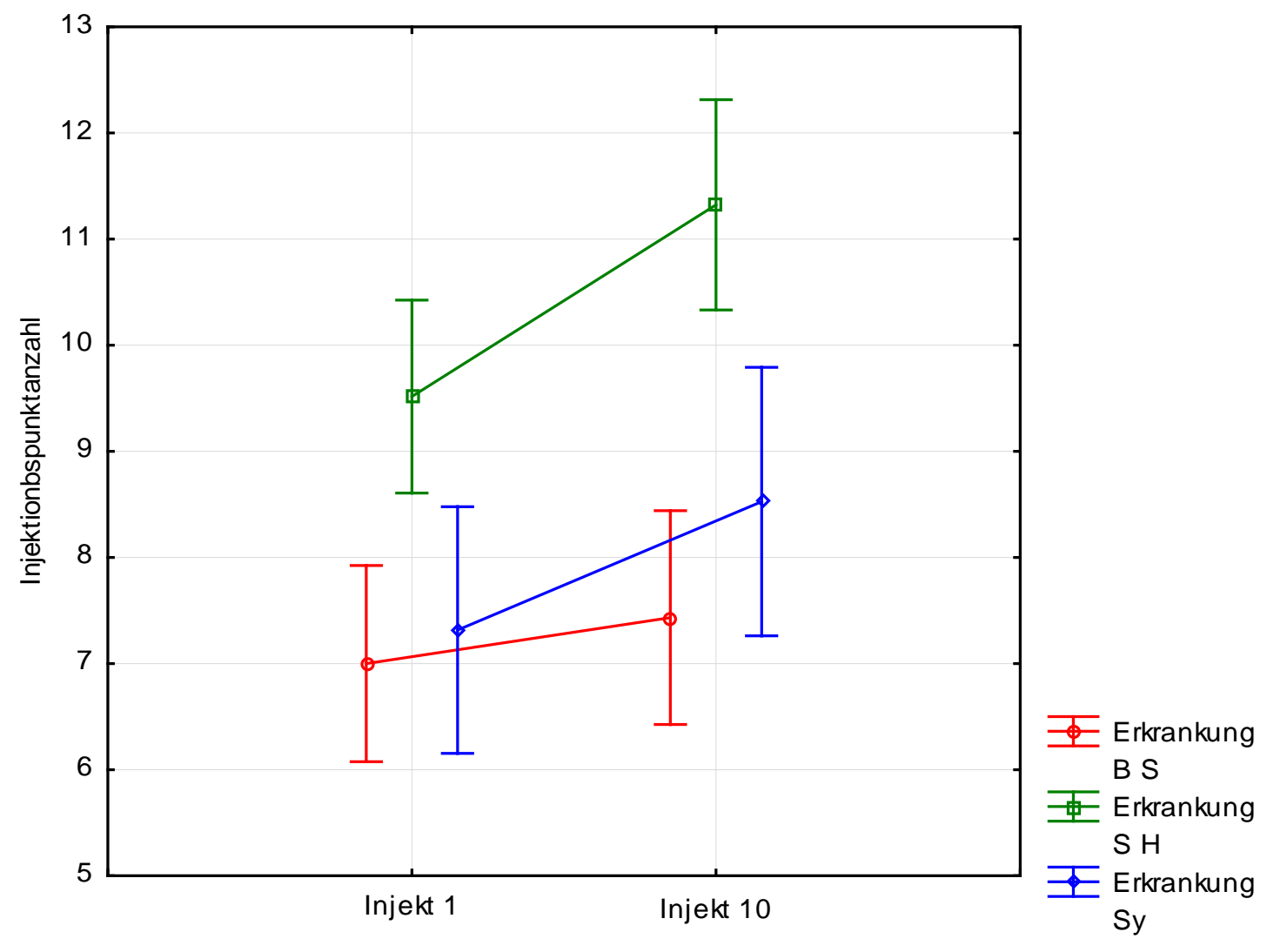

Abbildung 27: Mittlere Injektionspunktanzahl mit Konfidenzintervall zum ersten und zum zehnten Injektionszeitpunkt bei den Erkrankungsgruppen Blepharospasmus $(n=30)$, Spasmus facialis $(n=31)$, Synkinesien $(n=19)$.

\begin{tabular}{|c|c|c|}
\hline Faktor & p-Wert & Interpretation \\
\hline Erkrankungsgruppe & $\leq 0,01$ & signifikant \\
\hline Injektionseffekt & $\leq 0,01$ & signifikant \\
\hline Wechselwirkung & $\leq 0,04$ & signifikant \\
\hline
\end{tabular}

Tabelle 14: Ergebnisse in Form des p-Wertes der ANOVA mit Messwiederholungen, wobei die Zeitpunkte 1 und 10 betrachtet werden. 


\begin{tabular}{|c|c|c|c|c|c|}
\hline $\begin{array}{l}\text { Erkrankungsgruppe } \\
\qquad \mathbf{n}=\mathbf{8 0}\end{array}$ & \multicolumn{2}{|c|}{$\begin{array}{l}\text { Injektionspunkt- } \\
\text { anzahl }\end{array}$} & SD & $\begin{array}{c}\text { p-Wert } \\
\text { (adjustiert) }\end{array}$ & Interpretation \\
\hline \multirow{2}{*}{$\begin{array}{c}\text { Blepharospasmus } \\
n=30\end{array}$} & 1 & 7 & 1,3 & \multirow{2}{*}{$\leq 0,1$} & \multirow{2}{*}{$\begin{array}{c}\text { nicht } \\
\text { signifikant }\end{array}$} \\
\hline & 10 & 7,4 & 1,2 & & \\
\hline \multirow{2}{*}{$\begin{array}{l}\text { Spasmus facialis } \\
\qquad n=31\end{array}$} & 1 & 9,5 & 3,3 & \multirow{2}{*}{$\leq \mathbf{0 , 0 1}$} & \multirow{2}{*}{ signifikant } \\
\hline & 10 & 11,3 & 3,6 & & \\
\hline \multirow{2}{*}{$\begin{array}{c}\text { Synkinesien } \\
n=19\end{array}$} & 1 & 7,3 & 2,4 & \multirow{2}{*}{$\leq \mathbf{0 , 0 3}$} & \multirow{2}{*}{ signifikant } \\
\hline & 10 & 8,5 & 2,8 & & \\
\hline
\end{tabular}

Tabelle 15: Mittelwert der Anzahl der Injektionspunkte (Standardabweichung) in den Behandlungsgruppen zum Zeitpunkt der ersten und zehnten Behandlung und deren Signifikanz.

( $\mathrm{n}=$ Anzahl der Patienten zum Injektionszeitpunkt)

In Abbildung 28, die die 1. und 20. Behandlung darstellt, zeigt sich auch weiterhin die Tendenz, dass bei der Blepharospasmus-Gruppe die Anzahl der Punkte konstant bleibt (7,5 mit SD 0,6). Der höchste Anstieg ist in der Synkinesie-Gruppe zu sehen. Die Anzahl der Punkte (Standardabweichung) stieg von 6,3 (1,2) auf 9,8 (2) Punkte. In der Spasmus facialisGruppe betrug der Zuwachs von 10,2 (2,5) auf 13,2 (4,3) Injektionspunkte bei der 20. Behandlung. Der Mittelwertunterschied für die beiden Gruppen ist statistisch signifikant. Die Anzahl der Patienten betrug 41 zu diesem Behandlungszeitpunkt (siehe Tabelle 17). 


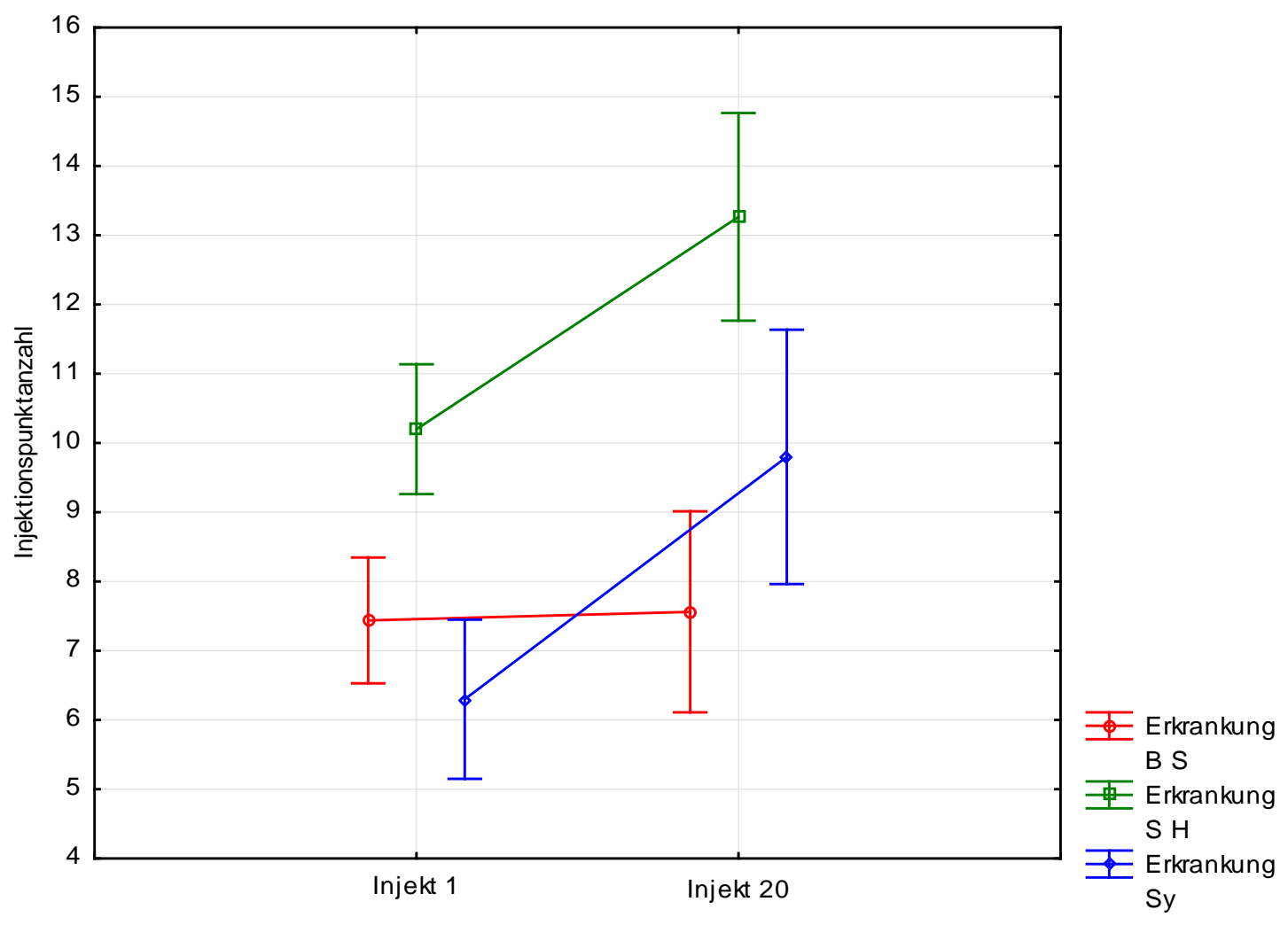

Abbildung 28: Mittlere Injektionspunktanzahl mit Konfidenzintervall zum ersten und zwanzigsten Injektionszeitpunkt bei den Erkrankungsgruppen Blepharospasmus $(n=16)$, Spasmus facialis $(n=15)$, Synkinesien $(n=10)$.

\begin{tabular}{|c|c|c|}
\hline Faktor & p-Wert & Interpretation \\
\hline Erkrankungsgruppe & $\leq 0,01$ & signifikant \\
\hline Injektionseffekt & $\leq 0,01$ & signifikant \\
\hline Wechselwirkung & $\leq 0,01$ & signifikant \\
\hline
\end{tabular}

Tabelle 16: Ergebnisse in Form des p-Wertes der ANOVA mit Messwiederholungen, wobei die Zeitpunkte 1 und 20 betrachtet werden. 


\begin{tabular}{|c|lr|c|c|c|}
\hline $\begin{array}{c}\text { Erkrankungsgruppe } \\
\mathbf{n}=\mathbf{4 1}\end{array}$ & \multicolumn{2}{|c|}{$\begin{array}{c}\text { Injektionspunkt- } \\
\text { anzahl }\end{array}$} & SD & $\begin{array}{c}\text { p-Wert } \\
\text { (adjustiert) }\end{array}$ & Interpretation \\
\hline $\begin{array}{c}\text { Blepharospasmus } \\
\mathbf{n}=\mathbf{1 6}\end{array}$ & 1 & 7,4 & 1 & $\leq 1$ & nicht signifikant \\
\cline { 2 - 4 } & 20 & 7,5 & 0,6 & signifikant \\
\hline $\begin{array}{c}\text { Spasmus facialis } \\
\mathbf{n}=\mathbf{1 5}\end{array}$ & 1 & 10,2 & 2,5 & $\leq \mathbf{0 , 0 3}$ & signifikant \\
\cline { 2 - 4 } $\begin{array}{c}\text { Synkinesien } \\
\mathbf{n}=\mathbf{1 0}\end{array}$ & 1 & 13,2 & 4,3 & \\
\cline { 2 - 4 } & 20 & 9,3 & 1,2 & $\leq \mathbf{0 , 0 1}$ & \\
\hline
\end{tabular}

Tabelle 17: Mittelwert der Anzahl der Injektionspunkte (Standardabweichung) in den Behandlungsgruppen zum Zeitpunkt der ersten und zwanzigsten Behandlung und deren Signifikanz.

( $\mathrm{n}=$ Anzahl der Patienten zum Injektionszeitpunkt)

In der Gruppe mit 30. Injektionen bleibt die Blepharospasmus-Gruppe ohne Veränderung der mittleren Anzahl der Injektionspunkte (7,7 mit SD 0,4). Die Patienten in der SynkinesieGruppe erfuhren auch zu diesem Behandlungszeitpunkt den höchsten Anstieg (von 6,5 auf 12,8 mit SD 1,1-1,6). In der Spasmus facialis-Gruppe kam es zu einem Anstieg der Punkte von 10,3 auf 14,3 (3,1-3,9). In dieser Untersuchungsgruppe befanden sich 25 Patienten (siehe Abb. 29 und Tabelle 19). 


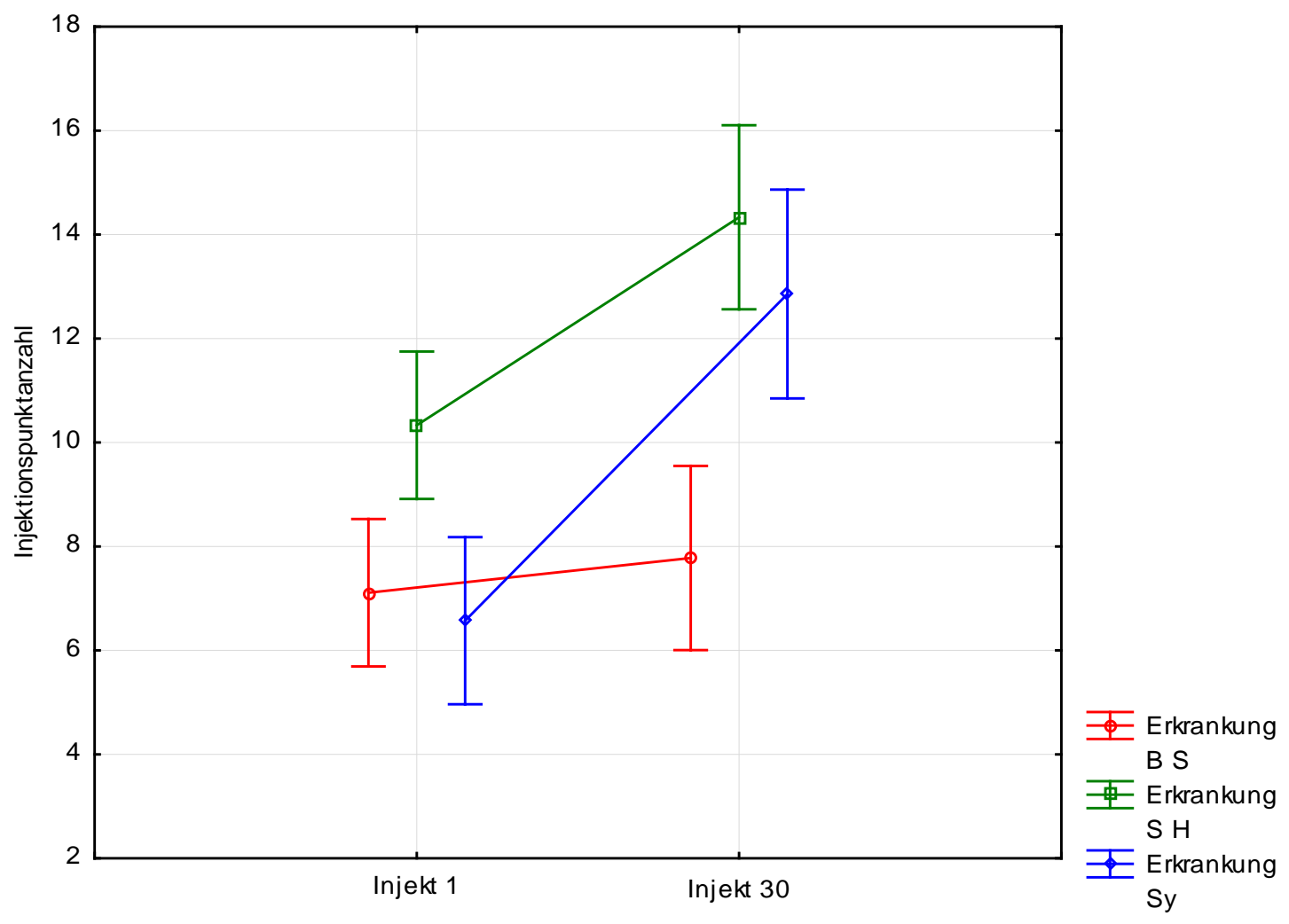

Abbildung 29: Mittlere Injektionspunktanzahl mit Konfidenzintervall zum ersten und dreißigsten Injektionszeitpunkt bei den Erkrankungsgruppen Blepharospasmus ( $n=9)$, Spasmus facialis $(n=9)$, Synkinesien $(n=7)$.

\begin{tabular}{|c|c|c|}
\hline Faktor & p-Wert & Interpretation \\
\hline Erkrankungsgruppe & $\leq 0,01$ & signifikant \\
\hline Injektionseffekt & $\leq 0,01$ & signifikant \\
\hline Wechselwirkung & $\leq 0,01$ & signifikant \\
\hline
\end{tabular}

Tabelle 18: Ergebnisse in Form des p-Wertes der ANOVA mit Messwiederholungen, wobei die Zeitpunkte 1 und 30 betrachtet werden. 


\begin{tabular}{|c|c|c|c|c|c|}
\hline $\begin{array}{l}\text { Erkrankungsgruppe } \\
\qquad n=25\end{array}$ & Inje & Inkt- & SD & $\begin{array}{c}\text { p-Wert } \\
\text { (adjustiert) }\end{array}$ & Interpretation \\
\hline \multirow{2}{*}{$\begin{array}{l}\text { Blepharospasmus } \\
\qquad \mathbf{n}=9\end{array}$} & 1 & 7,1 & 0,7 & \multirow{2}{*}{$\leq 0,1$} & \multirow{2}{*}{ nicht signifikant } \\
\hline & 30 & 7,7 & 0,4 & & \\
\hline \multirow{2}{*}{$\begin{array}{l}\text { Spasmus facialis } \\
\qquad \mathbf{n}=9\end{array}$} & 1 & 10,3 & 3,1 & \multirow{2}{*}{$\leq \mathbf{0 , 0 3}$} & \multirow{2}{*}{ signifikant } \\
\hline & 30 & 14,3 & 3,9 & & \\
\hline \multirow{2}{*}{$\begin{array}{l}\text { Synkinesien } \\
\quad n=7\end{array}$} & 1 & 6,5 & 1,1 & \multirow{2}{*}{$\leq \mathbf{0 , 0 1}$} & \multirow{2}{*}{ signifikant } \\
\hline & 30 & 12,8 & 1,6 & & \\
\hline
\end{tabular}

Tabelle 19: Mittelwert der Anzahl der Injektionspunkte (Standardabweichung) in den Behandlungsgruppen zum Zeitpunkt der 1. und der 30. Behandlung und deren Signifikanz.

( $\mathrm{n}=$ Anzahl der Patienten zum Injektionszeitpunkt)

Die Tendenz hat sich in der 40. Behandlung nicht geändert. Die Blepharospasmus-Gruppe bleibt im Durchschnitt (Standardabweichung) mit 7,7 (0,5) Injektionspunkten weiterhin ohne Veränderung. Die Synkinesie-Gruppe steigt von 6 (0) auf 12 (1) Injektionspunkte und die Spasmus facilais-Gruppe von 11,5 (2,1) auf 17 (0) Injektionspunkte pro Behandlung. Dieser Anstieg ist in den beiden Gruppen statistisch signifikant (siehe Abb. 30 und Tabelle 21).

In der Analyse konnte damit gezeigt werden, dass in der Synkinesie-Gruppe und Spasmus facialis-Gruppe nicht nur die Dosis über die Zeit sondern auch die Anzahl der Injektionspunkte im Mittel angestiegen ist. In der Blepharospasmus-Gruppe ist die Anzahl der Punkte über die Behandlungsjahre konstant geblieben, wobei die mittlere Gesamtdosis angestiegen ist. 


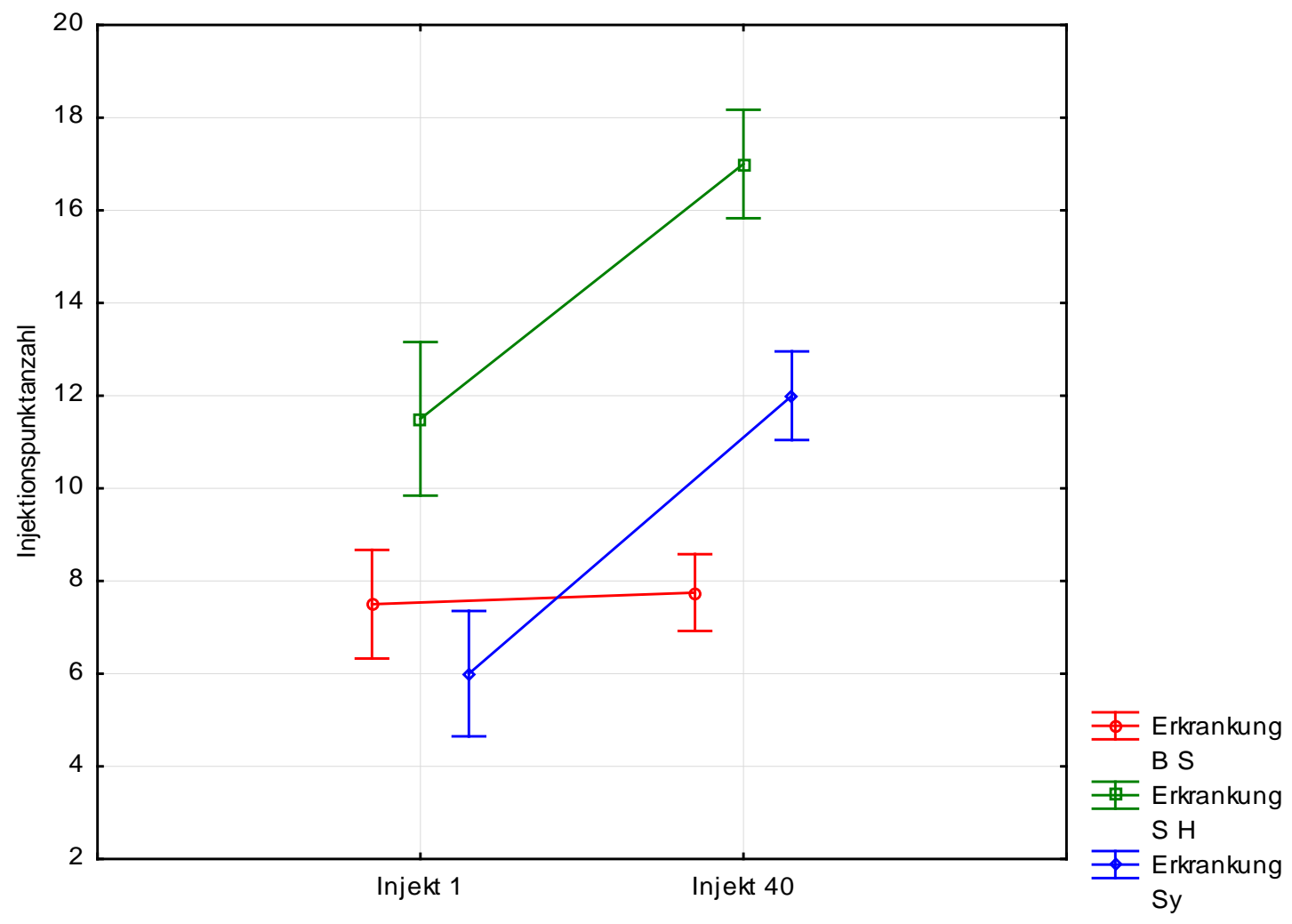

Abbildung 30: Mittlere Injektionspunktanzahl mit Konfidenzintervall zum ersten und vierzigsten Injektionszeitpunkt bei den Erkrankungsgruppen Blepharospasmus $(n=4)$, Spasmus facialis $(n=2)$, Synkinesien $(n=3)$.

\begin{tabular}{|c|c|c|}
\hline Faktor & p-Wert & Interpretation \\
\hline Erkrankungsgruppe & $\leq 0,01$ & signifikant \\
\hline Injektionseffekt & $\leq 0,01$ & signifikant \\
\hline Wechselwirkung & $\leq 0,01$ & signifikant \\
\hline
\end{tabular}

Tabelle 20: Ergebnisse in Form des p-Wertes von der ANOVA mit Messwiederholungen, wobei die Zeitpunkte 1 und 40 betrachtet werden. 


\begin{tabular}{|c|cc|c|c|c|}
\hline $\begin{array}{c}\text { Erkrankungsgruppe } \\
\mathbf{n}=\mathbf{9}\end{array}$ & \multicolumn{2}{|c|}{$\begin{array}{c}\text { Injektionspunkt- } \\
\text { anzahl }\end{array}$} & SD & $\begin{array}{c}\text { p-Wert } \\
\text { (adjustiert) }\end{array}$ & Interpretation \\
\hline $\begin{array}{c}\text { Blepharospasmus } \\
\mathbf{n}=\mathbf{4}\end{array}$ & 1 & 7,5 & 0,5 & $\leq 1$ & nicht signifikant \\
\cline { 2 - 4 } & 40 & 7,7 & 0,5 & signifikant \\
\hline $\begin{array}{c}\text { Spasmus facialis } \\
\mathbf{n}=\mathbf{2}\end{array}$ & 1 & 11,5 & 2,1 & \multirow{2}{*}{$\leq \mathbf{0 , 4 8}$} & signifikant \\
\cline { 2 - 4 } & 40 & 17,0 & 0,0 & \\
\hline $\begin{array}{c}\text { Synkinesien } \\
\mathbf{n}=\mathbf{3}\end{array}$ & 1 & 6 & 0,0 & $\mathbf{0 0 , 0 1}$ & \\
\cline { 2 - 4 } & 40 & 12 & 1,0 & & \\
\hline
\end{tabular}

Tabelle 21: Mittelwert der Anzahl der Injektionspunkte (Standardabweichung) in den Behandlungsgruppen zum Zeitpunkt der ersten und der vierzigsten Behandlung und deren Signifikanz.

( $\mathrm{n}=$ Anzahl der Patienten zum Injektionszeitpunkt)

\subsection{Dosis-Dynamik der Injektionspunkte bei den fazialen Dyskinesien}

Man kann in Abbildung 31 die Abhängigkeit der mittleren Dosis pro Punkt über die Anzahl der Injektionen ablesen. In der Gruppe der Patienten mit Blepharospasmus beobachtet man eine deutliche Steigerung der durchschnittlichen Dosis pro Injektionspunkt im Vergleich zu den anderen Erkrankungs-Gruppen. Der Anstieg resultiert aus der Erhöhung der Dosis pro Punkt, da die Injektionspunktanzahl über die Jahre konstant blieb. In der Gruppe der Patienten mit Spasmus facialis und Synkinesien ist die Steigerung der Dosis pro Injektionspunkt im Vergleich zu Blepharospasmus-Gruppe gering. Man beobachtet jedoch hier einen deutlicheren Zuwachs an Injektionspunkten über die Jahre. Die Dosis Steigerung resultiert hier von der Zunahme der Anzahl der Injektionspunkte über die Behandlungsjahre.

In dieser Analyse wurde auch die Entwicklung der Dosis am Injektionsort im Detail genau betrachtet. Die Abbildungen und Beschreibungen sind dem Anhang zu entnehmen. 


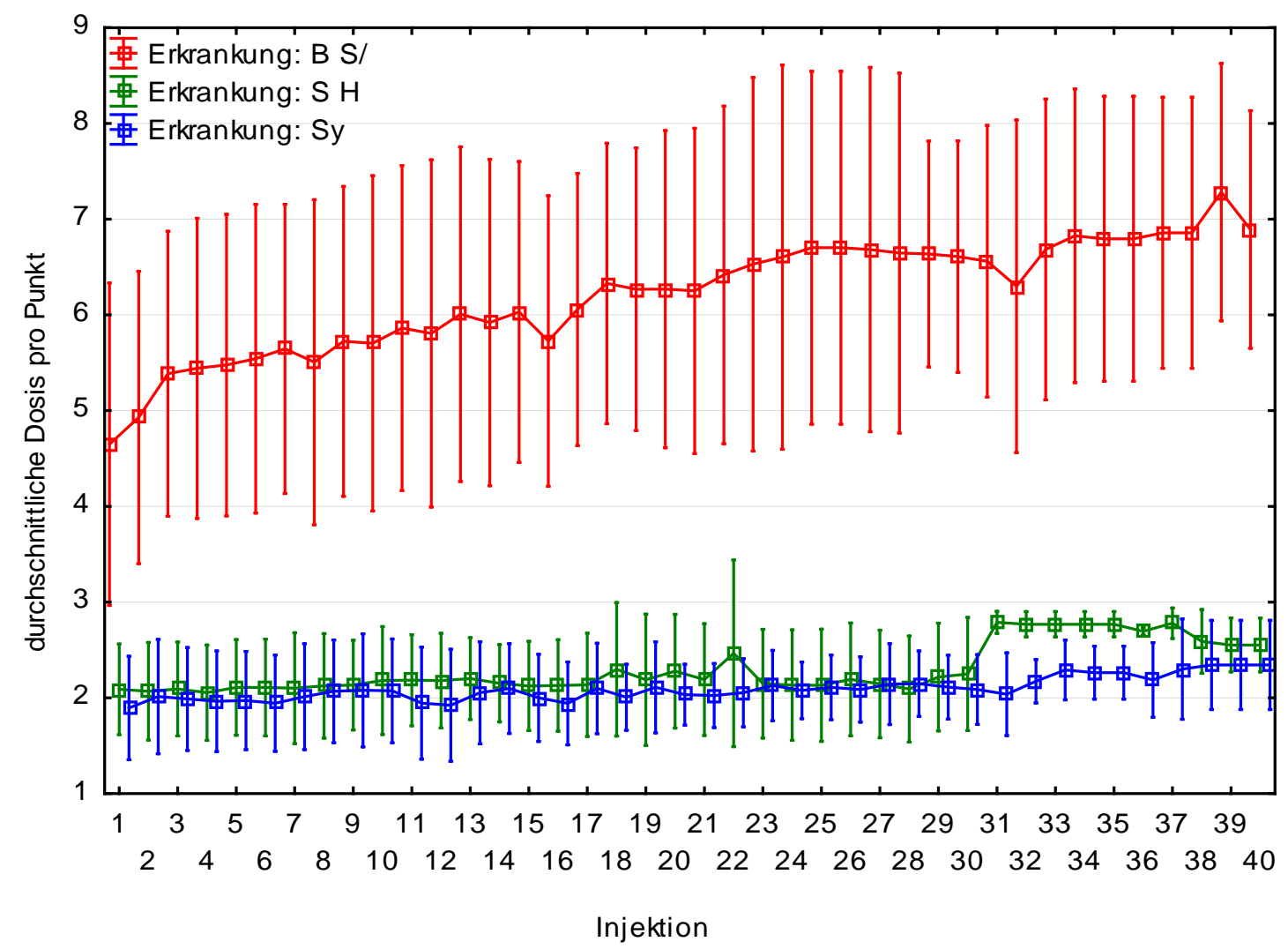

Abbildung 31: Entwicklung der durchschnittlichen Dosis pro Injektionspunkt mit Standardabweichung über die Zeit. 


\subsection{Nebenwirkungen der Botulinumtoxin-Therapie}

Bei allen drei Erkrankungs-Gruppen wurden insgesamt 1862 Behandlungen durchgeführt, 690 und 692 Behandlungen bekamen Patienten mit Blepharospasmus und Spasmus facialis. In der Gruppe mit Synkinesien wurden 480 Injektionen durchgeführt. Das Nebenwirkungsprofil fiel in jeder Behandlungsgruppe unterschiedlich aus. Während die häufigste Nebenwirkung bei Patienten mit Blepharospasmus mit 1,7\% (12 von 690) die Ptosis ist, ist das bei Patienten mit Spasmus facialis der hängende Mundwinkel mit 1,5\% (10 von 692). Bei Patienten mit Synkinesien ist die häufigste Nebenwirkung der Lagophthalmus mit 1,5\% (7 von 480). Die Ptosis ist auch stark in den anderen beiden Gruppen mit jeweils 0,6\% vertreten. Der Lagophthalmus ist bei Patienten mit Blepharospasmus und Spasmus facialis mit 0,9\% bzw. 0,7\% eine der häufigeren Nebenwirkungen. Ebenfalls häufig tritt eine Sicca-Symptomatik bei Patienten mit Blepharospasmus und Synkinesien auf (0,6\%; 1\%). In der Behandlungsgruppe des Blepharospasmus war auch die Epiphora (1,3\%) zu verzeichnen. Etwas seltener tritt das Hämatom als Nebenwirkung auf. Häufiger gab es Hämatome bei Patienten, die blutverdünnende Medikamente zum Zeitpunkt der Injektion eingenommen haben.

Betrachtet man alle drei Behandlungsgruppen zusammen, lässt sich die Aussage treffen, dass die Nebenwirkungsrate insgesamt gering ausfiel (siehe Tabelle 22).

\begin{tabular}{|c|c|c|c|c|c|c|}
\hline \multirow[b]{2}{*}{ Erkrankung } & \multicolumn{6}{|c|}{ Nebenwirkung } \\
\hline & Ptosis & $\begin{array}{l}\text { Lagoph- } \\
\text { thalmus }\end{array}$ & $\begin{array}{c}\text { Sicca- } \\
\text { Symptomatik } \\
\end{array}$ & Epiphora & $\begin{array}{l}\text { Hängender } \\
\text { Mundwinkel }\end{array}$ & Hämatom \\
\hline $\begin{array}{l}\text { Blepharo- } \\
\text { spasmus }\end{array}$ & 1,7 & 0,9 & 0,6 & 1,3 & & 1 \\
\hline $\begin{array}{l}\text { Spasmus } \\
\text { facialis }\end{array}$ & 0,6 & 0,7 & 0,15 & 0,3 & 1,5 & 0,3 \\
\hline Synkinesien & 0,6 & 1,5 & 1 & 0,2 & 0,4 & 0,4 \\
\hline Insgesamt & 1 & 1 & 0,6 & 0,7 & 0,7 & 0,6 \\
\hline
\end{tabular}

Tabelle 22: Nebenwirkungsprofil in \%-Angaben bei Patienten mit Blepharospasmus $(\mathrm{n}=690)$, Spasmus facialis $(\mathrm{n}=692)$, mit Synkinesien $(\mathrm{n}=480)$ und bei allen Gruppen zusammen (insgesamt $\mathrm{n}=1862$ ). 


\section{Diskussion}

\subsection{Wirkung von Botulinumtoxin A bei fazialen Dyskinesien}

Die klinische Wirkung von Botulinumtoxin A auf den jeweils behandelten Muskelbereich ist keine komplette Paralyse, sondern eine „Schwächung“ der Muskulatur bzw. eine Minderung der bestehenden Symptomatik. Die gewollte Verminderung der Muskelkontraktionen resultiert aus der spezifischen Hemmung der cholinergen Nervenendigungen an der neuromuskulären Endplatte. Tatsächlich kommt es jedoch zu einem völligen Sistieren der fehlerhaften Innervation für einen gewissen Zeitraum (Roggenkämper et al. 1991). Die Wirkung setzt zeitlich verzögert ein und der Wirkungsbeginn ist individuell unterschiedlich. Meist kommt es innerhalb von 7 bis 14 Tagen zu einem Eintritt der Wirkung. Das Ansprechen auf die injizierte Substanzmenge kann auch interindividuell erheblich variieren. Gleiche Dosen der Substanz können sowohl innerhalb eines Patientenkollektivs als auch bei demselben Patienten zu unterschiedlichen Injektionszeitpunkten einen differenten „Lähmungsgrad“ hervorrufen. Aus diesem geschilderten Grund ist die Dosisanpassung an das klinische Bild und an das Gespräch über die subjektiven Beschwerden des Patienten gekoppelt, um den maximalen Therapieerfolg zu erzielen (Roggenkämper 1994).

Die prinzipielle therapeutische Wirksamkeit des Botulinumtoxin A für die Indikationen Blepharospasmus und Spasmus hemifacialis wurde in vielen Studien belegt. Die subjektive Besserung der Symptomatik betrug zwischen 79\% bis 98,6\% (Yoshimura et al. 1992, Wang und Jankovic 1998, Jost und Kohl 2001, Defazio et al. 2002, Hsiung et al. 2002, Cannon et al. 2010). In einer Placebo-kontrollierten Studie an Patienten mit Spasmus hemifacialis wurde die Wirksamkeit des Botulinumtoxins A unabhängig von der Anfangsdosis nachgewiesen (Yoshimura et al. 1992). Eine Studie von Scott et al. (1985) verglich die Wirkung des Botulinumtoxins bei Blepharospasmus-Patienten mit einer Natrium-Chloridlösung, um die Wirksamkeit des Neurotoxins Botox ${ }^{\circledR}$ zu beweisen.

Für unsere Untersuchung haben wir diejenigen Patienten ausgewählt, die auf das Toxin angesprochen haben. Im Laufe der Behandlung sollte die geeignete Dosis gefunden werden. 


\subsection{Therapieintervalle}

Von großem Interesse in zahlreichen Arbeiten waren ebenso die zeitlichen Abstände zwischen den einzelnen Behandlungen bzw. die durchschnittliche Dauer der Wirkung der Therapie mit BTX/A.

Man beobachtete bei zahlreichen Analysen die Tendenz, dass die zeitlichen Abstände/Dauer der Wirkung für Patienten mit Spasmus facialis größer waren als die in der BlepharospasmusGruppe. Im Durchschnitt betragen in der Literatur die Zeiten zwischen den Behandlungen 13,1-14,9 Wochen für Blepharospasmus-Patienten und 15,4-16,3 Wochen für Patienten mit Spasmus facialis (Dutton und Buckley 1988; Frueh und Musch 1986; Mauriello und Aljian 1991, Snir et al. 2003, Vogt et al. 2008).

Gill und Kraft (2010) haben herausgefunden, dass bei Patienten mit Blepharospasmus, die 30 und mehr Behandlungen mit BTX/A bekommen haben, die zeitlichen Abstände zwischen den Behandlungen signifikant abgenommen haben (13,5 Wochen in den ersten 10 Behandlungen und 11,4 Wochen in den letzten 10 Behandlungen). In der Spasmus-facialis-Gruppe sind die Abstände zwischen den Behandlungen jedoch gleich geblieben (12,4 Wochen).

Kollewe et al. (2010) haben Patienten mit einem Spasmus facialis und Synkinesien jeweils in zwei Gruppen eingeteilt. Diese wurden mit Botox ${ }^{\circledR}$ oder Dysport ${ }^{\circledR}$ (im Lösungsverhältnis 1:2,56) im Durchschnitt 6 Jahre behandelt. Die Dauer der Wirkung betrug 12 Wochen in der Spasmus-facialis-Gruppe sowohl bei der Behandlung mit Botox ${ }^{\circledR}$ als auch mit Dysport ${ }^{\circledR}$. In der Synkinesien-Gruppe betrug die Zeit 11 Wochen für Botox ${ }^{\circledR}$ und 12 Wochen für Dysport ${ }^{\circledR}$. Dieser Unterschied war statistisch nicht signifikant.

Cannon et al. (2010) untersuchten eine Gruppe von 64 Patienten mit einem Blepharospasmus (30 Patienten) und mit einem Spasmus facialis (34 Patienten). Der Beobachtungszeitrum betrug 7,5 Jahre. Sie haben zeigen können, dass zwischen den beiden Gruppen ein statistisch signifikanter Unterschied in zeitlichen Abständen zwischen den Behandlungen existiert (11 Wochen versus 21 Wochen). Dabei hatte die Blepharospasmus-Gruppe signifikant häufiger eine Behandlung erhalten (25 versus 17 Behandlungen) als die Gruppe mit einem Spasmus facialis.

Diese Beobachtung ließ sich auch in der vorliegenden Analyse grundsätzlich bestätigen. Nach eigenen Berechnungen sind die zeitlichen Intervalle jedoch bei Patienten mit Blepharospasmus und Spasmus facialis in unserem Kollektiv größer. In unserem Patientengut betrugen 
die durchschnittlichen Zeitintervalle 4,5 Monate (19 Wochen) für die BlepharospasmusGruppe und 5,5 Monate (23 Wochen) für Patienten mit einem Spasmus facialis. Die Patienten hatten anhand der subjektiven Beschwerdesymptomatik zu entscheiden, wann der nächste Injektionstermin stattfinden sollte. In beiden Gruppen befanden sich Patienten die zwischen den Behandlungen längere Pausen hatten. In der Blepharospasmus-Gruppe sind es 4 Patienten die nach einem Jahr und 3 Monaten und 2 Patienten die nach einem Jahr zur erneuten Injektion vorstellig wurden. Jeweils ein Patient hatten drei Jahre und 6 Monate, vier Jahre und 5 Monate und sogar fünf Jahre Pause bis zur erneuten Injektion.

In der Gruppe mit einem Spasmus facialis gab es 7 Patienten, die bis zu der nächsten Injektion ein Jahr gewartet haben. Zwei Patienten hatten eine Pause von einem Jahr und 7 Monaten, ein Patient von einem Jahr und 10 Monaten und 4 Patienten kamen zu der nächsten Behandlung erst nach vollen zwei Jahren. Diese oben genannten Abstände sind jedoch nur vereinzelte Spitzenwerte. Ein Patient der vorliegenden Analyse kam insgesamt jedoch durchschnittlich nur alle 15 Monate zu den Injektionen. Dies ist im Hinblick auf die aktuelle Datenlage besonders, und mag somit ein Grund dafür sein, warum die zeitlichen Abstände in unserer Analyse von denen der anderen Autoren mitunter deutlich abweichen.

Die Intervalle zwischen den Behandlungen in der Patientengruppe mit Synkinesien betrugen bei anderen Autoren zwischen 16,6 Wochen (Laskawi et al. 1994) und 20,4 Wochen (Roggenkämper et al. 1991). Ito et al. (2007) konnten feststellen, dass bei Patienten mit Synkinesien, die mit Botox ${ }^{\circledR}$ behandelt worden waren, durchschnittlich alle 14 Wochen reinjiziert werden musste. Eine durchschnittliche Wirkung der Therapie mit BTX/A (Dysport ${ }^{\circledR}$ ) von 2 Monaten wurde von Chua et al. (2004) beschrieben. Die Arbeitsgruppe von Boroojerdi et al. (1998) betrachteten eine Patientengruppe mit Synkinesien (10 Pat.), die insgesamt 32 Injektionen bekommen hatten. Die Wirkdauer betrug hier durchschnittlich 24 Wochen. Über ähnliche zeitliche Abstände wie bei den Arbeitsgruppen von Roggenkämper et al. (1991) und Boroojerdi et al. (1998) kann in unserem Patientengut für Patienten mit Synkinesien (durchschnittlich 21 Wochen) berichtet werden. 


\subsection{Dosisentwicklung}

Seit Beginn der Therapie mit Botulinumtoxin beschäftigten sich zahlreiche Studien mit der Suche nach einer geeigneten Dosis um die best mögliche Wirkung zu erzielen, die zeitlich optimalen Behandlungsabstände zu erreichen und die Nebenwirkungsrate möglichst gering zu halten.

In dieser retrospektiven Analyse war von besonderem Interesse, ob nach einer zunächst gefundenen mittleren Substanzmenge mit guter Wirkung auch bei Langzeitpatienten eine weitere Dosisänderung notwendig wird.

Die ermittelten Ergebnisse haben gezeigt, dass nach Berechnung einer durchschnittlichen Gesamtdosis pro Injektion und untersuchter Patientengruppe es zu einer geringen, jedoch stetigen Dosiserhöhung im zeitlichen Verlauf kam. Diese Erkenntnis war überraschend, weil nach subjektiven Einschätzungen primär ein konstanter Dosisverlauf nach anfänglicher Dosisanpassung zu erwarten war. Man muss jedoch bedenken, dass in dieser Betrachtung bei allen Patienten und in den Erkrankungsgruppen unterschiedliche Patientenanzahl zu den angegebenen Zeitpunkten vorhanden war. Die Patientenanzahl wurde bis zu der 40. Injektion kleiner.

Dutton und Buckley (1988) berichteten, dass die meisten Patienten mit Blepharospasmus und Spasmus facialis eine konstante Dosis in der Behandlungszeit erfahren haben. Bei Patienten, die jedoch nicht adäquat auf die Behandlung angesprochen hatten, eine Dosiserhöhung von 25,5 E auf 75 E pro Auge notwendig war, um eine Reduktion der Spasmen erzielen zu können. Die Arbeitsgruppe von Ainsworth und Kraft (1995) zeigte, dass sich eine Dosissteigerung um 50\% bei Patienten mit Blepharospasmus nach der 12. Behandlung abzeichnete. Bei Patienten mit Spasmus facialis war der Anstieg der Dosis nicht signifikant und blieb dann auch nach der 12. Behandlung (Injektion) konstant.

Defazio et al. (2002) haben in einer Verlaufsbeobachtung über 10 Jahre bei 65 Patienten mit Spasmus facialis feststellen können, dass die Dosis von BTX/A nach 10 Jahren die gleiche war, wie die Dosis, die zum Beginn der Therapie injiziert wurde. Die durchschnittliche Anfangsdosis im ersten Jahr der Behandlung betrug 17,3 E. Nach zehn Jahren waren es 16,0 E. Bei diesem Patientengut wurde jedoch nur im Bereich des Musculus orbicularis oculi mit durchschnittlich 3-4 Injektionspunkten behandelt. 
In dem in dieser Arbeit verwendetem Standardschema wurde periorbital mit 6 Punkten begonnen. Nach Wunsch und Klinik der Patienten wurden im Verlauf der Therapie auch andere betroffene Muskeln injiziert. Die Dosis am einzelnen Injektionsort oder Muskel wurde dem subjektiven Beschwerdegrad oder dem Nebenwirkungsprofil des Patienten angepasst. Dies bedeutet, dass die Dosis im Laufe der Behandlungsjahre sowohl vermindert als auch erhöht werden konnte.

Hsiung et al. (2002) haben zeigen können, dass eine Dosissteigerung von BTX/A nach dem zweiten und fünften Jahr bei Patienten mit Spasmus facialis stattgefunden hatte. Diese Veränderung war jedoch statistisch nicht signifikant. Die durchschnittliche Dosis betrug 29,4 E. Bei Patientin mit Blepharospasmus sank die Dosis zunächst vom ersten zum zweiten und bis zum vierten Jahr, um dann eine Dosiserhöhung in den folgenden Jahren zu erfahren. Die durchschnittliche Dosis hier war 51,5 E. Problematisch bei dieser Studie und deren Vergleichbarkeit mit unseren Ergebnissen ist jedoch das Fehlen eines konkreten Injektionsschemas mit Angaben zur Lokalisation und Dosis pro Injektionspunkt.

Die Ergebnisse der Studie von Vogt et al. (2008) zeigten ebenso einen nicht signifikanten Anstieg der Dosis von BTX/A (Behandlung sowohl mit Botox ${ }^{\circledR}$ als auch mit Dysport ${ }^{\circledR}$ ) im Patientengut mit Spasmus facialis im Untersuchungszeitraum von 6 Jahren. Hier lag die mittlere Dosis zu Beginn der Therapie bei 15 E. Nach 6 Jahren stieg die Dosis auf insgesamt 19,25 E. In der Patientengruppe mit Blepharospasmus betrug die mittlere Anfangsdosis 27,75 E und nach 6 Jahren Behandlung 38,75 E. Hier konnte eine statistisch signifikante Dosiserhöhung im Laufe der Therapiejahre nachgewiesen werden. Eine Aussage zu der Anzahl der Injektionspunkte an den Muskeln und die Angaben zur genauen Dosierung wurden jedoch auch hier nicht getroffen.

Snir et al. (2003) haben bei ihren Untersuchungen eine Abhängigkeit der verabreichten Dosis und der erwünschten Wirkung nachweisen können. Dabei konnte die Arbeitsgruppe zeigen, dass eine Dosiserhöhung gehäuft mit einer kürzeren Wirkdauer beim Blepharospasmus einherging (4,0 vs. 3,2 Monate). Im Gegensatz dazu zeigte die gleiche Arbeitsgruppe, dass eine Dosiserhöhung zu einer längeren Wirkung beim Spasmus facialis führte (3,8 vs. 4,1 Monate). Diese Arbeitsgruppe (Snir et al. 2003) beobachtete 27 Patienten mit Blepharospasmus und Spasmus facialis über vier Jahre (12 Behandlungen pro Patient) und fand dabei über die Jahre eine Dosiserhöhung. Sie hatten für beide Gruppen ein Behandlungsschema mit den zu injizierenden Punkten und der minimalen Dosis (1,25-2,5 E) festgelegt. Dieses Schema 
entspricht periorbital genau dem hier verwendeten Therapieschema. Jeder Patient, bei dem ein unzureichendes Ansprechen auf das Botulinumtoxin A stattgefunden hatte, erfuhr eine Dosiserhöhung um 50\% bei der nachfolgenden Behandlung. Jeder Patient mit Blepharospasmus bekam im Durchschnitt 16,0 E. Nach 9 Injektionen stieg die Dosis durchschnittlich auf 24,2 E. In der Gruppe mit Spasmus facialis hat die Dosiserhöhung beim einzelnen Patienten von 16,8 E auf 25,0 E schon nach 6,5 Behandlungen stattgefunden. Diese Dosissteigerung wurde vorgenommen, um das Ansprechen der Muskulatur auf das Toxin und die Intervalle zwischen den Behandlungen bei 3-4 Monaten konstant zu halten.

Gill und Kraft (2010) haben eine Gruppe von 18 Pat. mit einem Spasmus facialis und 16 Pat. mit einem Blepharospasmus, die 30 und mehr Behandlungen mit BTX/A bekommen haben, ausgewertet. Sie konnten feststellen, dass die durchschnittliche Dosis über die Jahre in der Blepharospasmus-Gruppe gleichgeblieben ist (74 E). Die Patienten mit einem Spasmus facialis erfuhren eine Dosiserhöhung, die jedoch statistisch nicht signifikant war (32,9 E versus 38,4 E). Erklärt wurde dieses mit der Erweiterung der Injektionsorte im mittleren Gesicht und der Perioralregion.

Cannon et al. (2010) konnten zeigen, dass Patienten mit einem Blepharospasmus eine höhere Dosis von BTX/A (Botox $\left.{ }^{\circledR}\right)$ benötigt haben als Patienten mit einem Spasmus facialis (12,23 Einheiten versus 16,2 Units). Im Unterschied zu unserer Analyse wurde hier nur die periokuläre Region in Betracht gezogen und nur auf einer Seite bei Patienten mit einem Blepharospasmus.

Die Analyse der Arbeitsgruppe von Kollewe et al. (2010) hat über die Dosisentwicklung bei Patienten mit Spasmus facialis und Synkinesien nach durchschnittlicher Behandlung von 6 Jahren mit BTX/A berichtet. Sowohl die Spasmus-facialis- als auch die Synkinesien-Patienten wurden in Gruppen eingeteilt, die Botox ${ }^{\circledR}$ oder Dysport ${ }^{\circledR}$ erhalten hatten. Es bestand kein signifikanter Unterschied in der durchschnittlichen Dosis zwischen den Erkrankungsgruppen oder den beiden Präparaten (Spasmus facialis Botox ${ }^{\circledR}$ : 22 E, Dysport ${ }^{\circledR}$ : 51 E; Synkinesien: Botox ${ }^{\circledR}: 19$ E, Dysport ${ }^{\circledR}: 45$ E). Sie konnten auch feststellen, dass 14\% aller Patienten eine Erhöhung der Dosis über die Zeit erfahren hatten. Dieses war mit dem Injektionsschema zu erklären. Es wurden 3-4 Punkte am M. orbicularis oculi und je nach Ausprägung der Symptomatik im Verlauf der Erkrankung auch zusätzliche andere Muskelregionen (M. zygomaticus major, buccinator, corrugator und frontalis) behandelt. Diese Vorgehensweise entspricht der Behandlung in unserer Auswertung. 
In der hier betrachteten Analyse betrug die mittlere Dosis bei der Blepharospasmus-Gruppe zunächst 33,3 E und stieg bis zu der 40. Injektion auf 53,4 E an (durchschnittliche Dosis bei beiden Augen). Wir konnten jedoch nur vier Patienten bis zu der 40. Injektion auswerten. Patienten mit Spasmus facialis begannen im Durchschnitt mit 20,5 E und bekamen bei der 40. Behandlung 43,4 E verabreicht. Nur zwei Patienten haben in dieser Gruppe 40 Injektionen erhalten. In dem verwendeten Behandlungsschema wurde keine standardisierte Dosiserhöhung bei unzureichendem Ansprechen auf das Toxin vorgenommen. Diese richtete sich in unserer Arbeit entgegen der beschriebenen Literatur bei jeder erneuten Behandlung nach dem subjektiven Beschwerdebild des Patienten bzw. nach dem Nebenwirkungsprofil.

Die ersten Erfahrungen mit der Behandlung der Synkinesien mit Botulinumtoxin A machte die Arbeitsgruppe von Roggenkämper et al. (1990). Hier wurde, wie auch bei den Patienten der HNO-Klinik Göttingen, das Medikament entsprechend der betroffenen Muskelgruppen und der Stärke der Synkinesien dosiert. Die nach Fazialisparese verminderte Lidschlusskraft wurde immer mitberücksichtigt und in dieser Studie gemessen. Die Zahl der Injektionsorte am Musculus orbicularis oculi variierte zwischen 6 und 10 Injektionspunkten. Die Dosis am Injektionsort betrug zwischen 1,25 E und 2,5 E. Der Beobachtungszeitraum war jedoch nur von der ersten bis zur zweiten Injektion. Die Angaben zur Gesamtdosis im Verlauf fehlten in dieser Analyse.

Vier Jahre später errechneten Laskawi et al. (1994) zusätzlich die Dosis von Botox ${ }^{\circledR}$ pro behandeltes Auge (zwischen 3,75- 23,75 E). Sie konnten ebenso feststellen, dass Patienten mit einem kompletten Lidschluss bei der pathologischen Mitbewegung einer höheren Dosis bedurften um zufriedenstellende Ergebnisse erzielen zu können.

Boroojerdi et al. (1998) haben bei 10 Patienten mit Synkinesien über eine durchschnittliche Dosis mit BTX/A (Dysport ${ }^{\circledR}$ ) von 75 Einheiten berichtet. Hier wurde im Mittel an 4 Punkten des M. orbicularis oculi injiziert.

Chua et al. (2004) untersuchten 5 Patienten mit Synkinesien. Sie konnten feststellen, dass eine durchschnittliche Dosis von BTX/A (Dysport ${ }^{\circledR}$ ) von 40 Einheiten, die in zwei Punkte am M. orbicularis oculi appliziert wurden, ausreichend war, um eine effektive Wirkung zu erzielen. Eine höhere Dosis hatte zu einer passageren Ptosis geführt.

Die low-dose-Behandlung der Patienten mit Synkinesien hat die Analyse von Ito et al. (2007) bestätigt. 7 Patienten bekamen BTX/A (Botox ${ }^{\circledR}$ ) am M. orbicularis oculi und M. orbicularis 
oris durchschnittlich an 5-6 Punkten injiziert. Die Gesamtdosis betrug im Mittel 5-6 Einheiten und die Dosis pro Injektionspunkt betrug 1 Einheit.

Diese Arbeitsgruppen postulieren, dass eine geringere Dosis, im Vergleich zu Patienten mit einem Blepharospasmus, ausreichend ist, um einen guten Effekt der Therapie zu erreichen.

Die durchschnittliche Gesamtdosis in unserem Patientenkollektiv mit Synkinesien betrug zwischen 14,3- 28,3 E, wobei auch hier die Dosis im perioralen und Platysmabereich mitberechnet wurde. In unserer Analyse konnten wir 19 Patientendaten auswerten. Bei 37\% der Erkrankten begannen wir ab der ersten Injektion mit der Behandlung der perioralen oder Platysmaregion. Diese Unterschiede können für die höhere mittlere Dosis in unseren Berechnungen verantwortlich sein.

In dieser Analyse konnte auch die Beobachtung gemacht werden, dass die Patientengruppe mit Blepharospasmus diejenige ist, in der mit der höchsten durchschnittlichen Anfangsdosis, nämlich mit 33,2 E, begonnen wurde. Bei Patienten, die an Spasmus facialis erkrankten, wurde im Mittel mit 20,5 E begonnen. Die geringste Anfangsdosis wurde mit 14,3 E bei Synkinesien appliziert.

In der hier verwendeten Analyse wurden die Behandlungsgruppen $\mathrm{zu}$ bestimmten Injektioszeitpunkten (1, 10, 20, 30, 40) untereinander verglichen. In dieser Betrachtung ist die Anzahl der Patienten zum untersuchten Zeitpunkt jedoch unterschiedlich. Die Patientenanzahl wird im Durchschnitt kleiner.

Ein durchschnittlicher Anstieg der mittleren Dosis zeichnet sich jedoch insgesamt in diesen Ergebnissen ab. Je mehr Behandlungen ein Patient bekam, umso höher war der Anstieg der mittleren Gesamtdosis.

\subsection{Dosiserhöhung und deren mögliche Ursachen}

Bis jetzt sind die Ursachen einer erforderlichen Dosissteigerung spekulativ bzw. nicht vollständig geklärt. Ein individueller Krankheitsverlauf mit unterschiedlicher Ausprägung der Symptomatik unter bestimmten Lebensumständen ist beispielsweise bekannt. Auch, dass die Intensität und Stärke der Verkrampfung sich während der Zeit progressiv entwickeln können. Diese Umstände können eine Dosisanpassung notwendig machen (Snir et al. 2003). Das Erleben der Erkrankung durch die Patienten und der Wunsch nach Optimierung des Therapieerfolges könnten auch einen relevanten Einfluss auf die Dosissteigerung haben. Man 
darf auch die Erfahrung des behandelnden Arztes nicht außer Acht lassen, denn seine praktische Erfahrung und Vertrautheit mit den Krankheitsbildern könnte einen Einfluss auf die verabreichte Dosis und Entscheidung über den Injektionsort haben.

Denkbar ist, dass sich die dystonen Aktivitäten auch auf die tiefer liegende Muskulatur ausbreiten könnten. Diese sind womöglich der Therapie weniger gut zugänglich, was zu einer Dosiserhöhung an der oberflächlichen Muskulatur führen kann (Vogt et al. 2008).

In dieser Analyse haben wir uns mit der Frage befasst, ob eine Dosisänderung über die Zeit von einer unterschiedlichen „Dosisentwicklung pro Punkt“ und/oder einer Änderung der „Anzahl der gesetzten Injektionspunkte“ hervorgerufen wird. In der aktuellen Literatur sind keine vergleichbaren Daten zu dieser Fragestellung zu finden. Dieser Aspekt kann für die untersuchten Erkrankungsgruppen jedoch für die Behandlung von Bedeutung sein. Es könnten krankheitsbezogene Rückschlüsse hinsichtlich der Notwendigkeit einer Therapieänderung im Sinne einer Dosisänderung am Injektionsort oder im Sinne einer Optimierung der Wirkung durch Behandlung anderer Areale gezogen werden. Im zeitlichen Verlauf der Botulinumtoxin-Behandlung könnten auch beide Veränderungen von Bedeutung sein, um einen optimalen Effekt der Therapie zu erreichen.

Eine der möglichen Erklärungen für die Steigerung der Gesamtdosis könnte der Anstieg der Behandlungspunkte im Therapieverlauf oder eine Erhöhung der Dosis pro Punkt sein. Die Anzahl der Injektionspunkte im periorbitalen Bereich bei Patienten mit Blepharospasmus beträgt zwischen 7 und 7,7 Punkten auf beiden Seiten und bleibt über die Behandlungsjahre konstant. Die Dosis pro Punkt steigt jedoch durchschnittlich in geringem Maße von 2,5 E auf 4,0 E. Im Unterschied zu den anderen Erkrankungen, ist die betroffene, fehlbewegte Muskelgruppe beim essentiellen Blepharospasmus relativ konstant, so dass sich die Dosissteigerung vor allem durch die Erhöhung der Dosis am Injektionsort ergibt. Aber auch hier gibt es die Möglichkeit mehr Injektionsorte zu injizieren (zusätzlich: Punkt kaudal des Musculus orbicularis oculi, Punkt im Musculus frontalis), damit eine gute Wirksamkeit erreicht werden kann, die jedoch für die durchschnittliche Dosissteigerung nicht relevant erscheint.

Wir haben jedoch bei dieser Patientengruppe bei der Berechnung der mittleren Gesamtdosis über die Jahre sämtliche Injektionspunkte miteinbezogen. Bei den Patienten mit MeigeSyndrom sind auch weitere Injektionspunkte im Gesichtsbereich hinzugekommen, die wir in diese Auswertung einfließen ließen. Es könnte daher eine allgemeine Tendenz zu einer 
Dosissteigerung über die Jahre mit beeinflusst haben. Dieses spiegelt jedoch nur den natürlichen Verlauf der Behandlung einer Krankheitsentwicklung und den jeweiligen Zustand der Ausprägung der Symptomatik wider.

Die Ergebnisse bei Patienten mit Spasmus facialis und Synkinesien zeigen eine ähnliche Tendenz bei jedoch unterschiedlichen Dosierungen und unterschiedlicher Entwicklung der Injektionspunktanzahl. In der Spasmus facialis-Gruppe kann man sagen, dass die Anzahl der injizierten Punkte stetig von 9 auf 17 Punkte ansteigt. Hier beobachteten wir aber eine gering ausgeprägte mittlere Dosiserhöhung pro Injektionspunkt. Der Anstieg der Dosis scheint in dieser Gruppe aus beiden untersuchten Effekten zustande zu kommen. Die Dosis im Augenbereich blieb über die Behandlungszeit aber relativ konstant, geringe Schwankungen (je nach Injektionsort gab es sowohl eine Verminderung als auch eine Erhöhung der Dosis) waren jedoch zu beobachten. Wurde im Bereich des Platysma injiziert, so blieben die Dosen ebenso konstant. Die größte Abweichung sahen wir im Bereich des Musculus zygomaticus und im perioralen Bereich. Aus unseren Daten konnte entnommen werden, dass sich die Behandlung besonders dieser Region nach dem Muster des Nebenwirkungsprofils richtete. Um das Herunterhängen des Mundwinkels auf einer betroffenen Seite zu vermeiden, wurde die Dosis eines Injektionspunktes auf ein oder zwei benachbarte Injektionsorte verteilt. Diese Tatsache könnte auch zu Veränderung der Injektionspunktanzahl geführt haben. Diese Daten konnten wir in keiner bisherigen Untersuchung finden und somit auch nicht mit unseren Daten vergleichen. Auch hier haben wir die mittlere Dosisentwicklung aller injizierten Punkte pro Injektion berücksichtigt.

In der Patientengruppe mit Synkinesien nach Fazialisdefektheilung fanden wir eine äußerst geringe mittlere Dosisminderung pro Injektionspunkt über die Zeit. Die Anzahl der Injektionspunkte ist jedoch von der ersten bis zu der vierzigsten Behandlung gestiegen (7-12). In dieser Gruppe scheint also das größere Behandlungsareal für die Dosiserhöhung verantwortlich zu sein.

Die klinische Ausprägung in der Synkinesien-Gruppe unterliegt einer großen Variabilität, so dass die Dosissteigerung aber auch teilweise Minderung durch die Veränderung der Injektionspunkte relevant zu sein scheint.

Die Erkrankungen zeigen somit kein einheitliches Bild der symptomatischen Beschwerden. Endogene oder exogene Faktoren können zu Exazerbationen der Erkrankung führen, so dass 
intraindividuelle Änderungen der Intensität der Spasmen vorkommen können. Dies kann zu unterschiedlichen Zeitpunkten eine Anpassung der Dosis notwendig machen, so dass wir keine einheitliche Dosiserhöhung bei unzureichenden oder nicht zufriedenstellenden Ergebnissen angewandt haben.

Ferner können im Laufe der Behandlung neutralisierende Antikörper gebildet werden. Nach derzeitigen Erkenntnissen können diese Antikörper nach 3-12 Injektionsserien zu einem partiellen antikörpervermittelten Therapieversagen führen. Häufig kommt es dann nach weiteren 2-3 Behandlungen zu einem kompletten Versagen der Therapie (Dressler und Benecke 2004). Dieses Versagen der Therapie ist aber nach der 14. Injektion laut der aktuellen Literatur unwahrscheinlich. Hsiung et al. (2002) berichteten jedoch über Einzelfälle, in denen noch nach 10 Jahren ein antikörpervermitteltes Therapieversagen stattgefunden hatte. Die Häufigkeit des Auftretens von Antikörpern schwankt in den Literaturangaben zwischen 2\% und 9 \% (Hsiung et al. 2002). Dieser Fakt korreliert mit der Höhe der Einzeldosis, der Häufigkeit der Behandlungen und möglicherweise mit der Kumulativdosis, die im Therapieverlauf angewendet wurde (Snir et al. 2003).

Es ist in dieser Betrachtung jedoch zu beachten, dass die Anzahl der Patienten über die Zeit abnimmt, so dass die Ergebnisse mit Vorsicht interpretiert werden sollten. Die Daten zeigen dennoch eine gewisse Tendenz, die in der praktischen Durchführung behilflich sein können. Es liegen jedoch relativ lange Beobachtungszeiträume (7-9 Jahre bei der 20. Injektion) mit größeren Patientenzahlen vor, was unsere Beobachtungen stützt.

\subsection{Anzahl der Injektionspunkte und die Dosisentwicklung am Injektionsort}

In unseren Ergebnissen kamen wir zum Schluss, dass die durchschnittliche Dosis über die Jahre stetig ansteigt. Von Interesse war es herauszufinden, ob die Erhöhung der Gesamtdosis an einer Dosiserhöhung der konstanten Punkte lag und/oder der Zunahme der Injektionsorte während der Behandlungsjahre. Es gibt nur wenige Studien über diese Zusammenhänge.

Das Schema der Injektion in unseren Behandlungsgruppen war sehr variabel. Die Anzahl der Injektionspunkte wurde anhand der sichtbaren und von der vom Patienten angegebenen Ausprägung der Symptomatik festgelegt. Es gab zwar ein festgelegtes Schema der Injektionsorte, die jedoch variabel eingesetzt wurden. Kamen die Patienten mit einer ausgeprägten Symptomatik im perioralen oder Platysmabereich zur nächsten Konsultation und wünschten 
Linderung auch dieser Beschwerdesymptomatik, haben wir diese Region mitbehandelt. Dieses unterscheidet unsere Analyse von vielen anderen, wo ein bestimmtes Schema eingesetzt und eingehalten wurde.

Die verwendete Dosis wurde an die Angaben der Patienten angepasst. Je stärker die Verkrampfungen vorhanden waren und je stärker das subjektive Leiden des Patienten war, umso höher wurde die Dosis am Injektionsort gewählt und appliziert.

Wir konnten feststellen, dass bei Patienten mit einem Blepharospasmus die Anzahl der Punkte über die Behandlungsjahre kaum angestiegen ist, die Dosis pro Injektionsort jedoch anstieg.

Die Anzahl der Injektionspunkte bei Patienten mit Synkinesien war in unserer Analyse über die Jahre zwischen 7 und 12, wobei der periorale und der Platysmabereich mitberücksichtigt wurden. Die Dosis pro Injektionspunkt liegt im Mittel, ähnlich der Arbeitsgruppe von Roggenkämper et al. (1990), zwischen 1,9 E und 2,5 E über alle Behandlungsjahre. Ähnliche Ergebnisse mit einer größeren Patientenanzahl zeigte eine Studie von Roggenkämper et al. (1994) vier Jahre später.

In unserer Analyse haben wir bei 58\% der Patienten die Injektionen im periokulären Bereich begonnen. Bei der letzten Behandlung waren die perioralen und/oder die Injektionspunkte im Platysmabereich fast immer zusätzlich vorhanden. Die Anzahl der Punkte und der Zeitpunkt ab welcher Injektionsanzahl die Punkte dazugekommen waren, war jedoch bei unseren Patienten sehr unterschiedlich.

Auch bei den Patienten mit einem Spasmus hemifacialis sind die Injektionsorte von 9 auf 17 über die gesamten Behandlungsjahre angestiegen. Bei 25\% (8 von 31 Pat.) der Patienten sind zusätzliche Injektionspunkte erst im späteren Behandlungsverlauf hinzugekommen, so dass auch hier der Anstieg der Injektionspunkte damit erklärt werden kann.

\subsection{Nebenwirkungen der Botulinumtoxin-Therapie}

Sowohl alle bekannten Studien, als auch diese Auswertungen zeigten, dass das Nebenwirkungsprofil von untergeordneter Bedeutung ist und die unerwünschten Wirkungen fast immer lokal auftreten. Alle auftretenden Effekte sind insgesamt vorübergehender Natur. Die Patienten empfinden sie meistens als nicht so störend, und deshalb sind sie selten ein Grund, die Therapie mit Botulinumtoxin abzubrechen. Es wurde in keiner Studie nachgewiesen, dass systemische Nebenwirkungen (wie z.B. ein anaphylaktischer Schock oder Todesfall) jemals 
auftraten. In unserem Patientenkollektiv hat keiner der Patienten aufgrund des Nebenwirkungsprofils die begonnene Therapie abgebrochen. Die häufigste unerwünschte Wirkung war in den meisten Untersuchungen die Ptosis. Die Angaben schwanken zwischen 7-38\%. Die Autoren beschreiben, dass eine höhere Rate an Ptosis mit der zuvor durchgeführten Erhöhung der Dosis am Auge korreliert. Die anderen beschriebenen Nebenwirkungen waren: SiccaSyndrom, Schwäche der mimischen Muskulatur, Diplopie und Lagophthalmus. Die ungewollten Symptome waren in den meisten Fällen 2-4 Wochen nach der Injektion nicht mehr vorhanden (Chen et al. 1996, Defazio et al. 2002, Yoshimura et al. 1992, Hsiung et al. 2002, Wang und Jankovic 1998, Dutton und Buckley 1988, Mauriello et al.1996a, b). Die Arbeitsgruppen von Roggenkämper et al. (1994) und Laskawi et al. (1994) berichteten über Epiphora als die häufigste Nebenwirkung (7-10\%).

Den Berechnungen dieser Analyse nach ist die Nebenwirkungsrate, bezogen auf alle Injektionsbehandlungen und Erkrankungsgruppe, insgesamt sehr gering. Bei Patienten mit Blepharospasmus ist die Ptosis mit 1,6\% und bei Patienten mit Spasmus facialis der hängende Mundwinkel mit 1,5\% die häufigste Nebenwirkung. Patienten mit Synkinesien klagten am häufigsten über einen Lagophthalmus (1,5\%).

\subsection{Schlussfolgerung}

Anhand bisheriger Erfahrungen und zahlreicher Analysen lässt sich die Aussage treffen, dass das Botulinumtoxin A das Mittel der Wahl zur Behandlung des Blepharospasmus und der Synkinesien nach Fazialisdefektheilung darstellt.

Beim Spasmus facialis gilt nach wie vor als kurativer Ansatz die mikrovaskuläre Dekompression. Aufgrund der möglichen Komplikationen, entscheiden sich nach unseren Erfahrungen die meisten Patienten jedoch für die regelmäßige Applikation von Botulinumtoxin A.

Wir konnten in unserer Analyse zeigen, dass die Botulinumtoxin-Dosis im Verlauf der Behandlungsjahre stetig ansteigt. Es gibt verschiedene Ansätze, die eine Erhöhung der Dosis zu erklären versuchen. Zum einen sind das die anfängliche Dosisanpassung, die zunehmende Intensität der Ausprägung der Krankheitsbilder, die Zunahme der Dosis am Injektionspunkt, und die Zunahme der Injektionspunkte im Behandlungsschema. Zum anderen ist es die 
mögliche Ausbildung neutralisierender Antikörper. Für den Patienten hat es jedoch, wenn die Therapie die gewünschte Wirkung zeigt, keine negativen Folgen.

Botulinumtoxin A ermöglicht ein nicht-invasives Vorgehen. Es zeichnet sich durch ein geringes Nebenwirkungsprofil aus. Zusätzlich ist die Zufriedenheit der Patienten und eine hohe Quote der subjektiven Besserung der Symptomatik nach der Behandlung ein Hinweis für eine sehr gute Wirksamkeit des Medikamentes. Ein sehr gutes Argument für die Behandlung besteht auch in der Beeinflussung der ästhetischen Integrität des Gesichtes. Ebenso bedeutend sind die funktionellen Vorteile nach der Therapie. Die Patienten sind kurzfristig wieder in der Lage ihre Augen zu öffnen, so dass ein gesteigertes Selbstvertrauen und eine positive Beeinflussung des psychosozialen Umfeldes gegeben sind. Außerdem wird erreicht, dass die Mimik der Patienten wieder entsprechend der emotionalen Situation angepasst werden kann. 


\section{Zusammenfassung}

Botulinumtoxin blockiert, als äußerst potenter Inhibitor cholinerger Synapsen, im Bereich der muskulären Endplatte sowie der Schweißdrüsen den ankommenden Impuls, indem es die Ausschüttung von Acetylcholin in der präsynaptischen Nervenendigung irreversibel hemmt. Die Wirkung ist jedoch von begrenzter Dauer, denn es bilden sich später neue Nervenendigungen, die neue synaptische Verbindungen herstellen und die neuronale Aktion wieder aufnehmen.

Die Botulinumtoxin-Injektion ist seit 1983 die Therapie der Wahl beim essentiellen Blepharospasmus, der fokalen, kranialen Dystonie. Auch beim Spasmus hemifacialis, trotz eines vorhandenen kurativen neurochirurgischen Verfahrens (mikrovaskuläre Dekompression) und bei Synkinesien kann diese Therapie als wirksame Therapie angesehen werden.

In der retrospektiven Langzeitbeobachtungsanalyse wurde der Verlauf der Dosisentwicklung bei Patienten mit Blepharospasmus, Spasmus hemifacialis und Synkinesien über die Zeit verfolgt. Die Patientengruppen wurden zusammen, getrennt und abhängig von der erhaltenen Behandlungsanzahl analysiert. In jedem dieser Fälle konnte eine Dosissteigerung beobachtet werden. Es gibt einige Gründe, die eine Dosiserhöhung über die Zeit erklären könnten. Das Ziel der Analyse war es herauszufinden, ob der Anstieg der Dosis mit einer Dosiserhöhung pro Injektionspunkt und/oder mit einer Zunahme der Injektionsorte über die Zeit zusammenhängt. Eine Zunahme der Injektionspunkte konnte insbesondere bei Patienten mit Spasmus hemifacialis und Synkinesien nachgewiesen werden, während bei Patienten mit Blepharospasmus vor allem eine geringe, aber kontinuierliche Dosissteigerung pro Injektionspunkt stattgefunden hat.

Die geringe Invasivität und das Fehlen schwerer und dauerhafter unerwünschter Wirkungen von Botulinumtoxin stellen die Vorteile gegenüber anderen, wenigen und doch nebenwirkungsreicheren Therapiemöglichkeiten dar. Die Wirkdauer von 3-5 Monaten ermöglicht die Nutzung von Botulinumtoxin Typ A zur passageren Reduktion und Linderung der störenden Spasmen. Es ist eine lebenslange Therapie, die die Erkrankten leider nicht heilen kann, jedoch sehr effizient die Verkrampfungen reduzieren kann. 
Wird Botulinumtoxin in regelmäßigen Abständen von 3 Monaten in adäquater Dosierung verabreicht, ist die Gefahr der Antikörperbildung bei diesen Erkrankungen gering. Diese Tatsache war nicht Gegenstand unserer Untersuchung, obwohl sie als möglicher Grund für eine Dosiserhöhung nicht vergessen werden sollte. 


\section{Anhang}

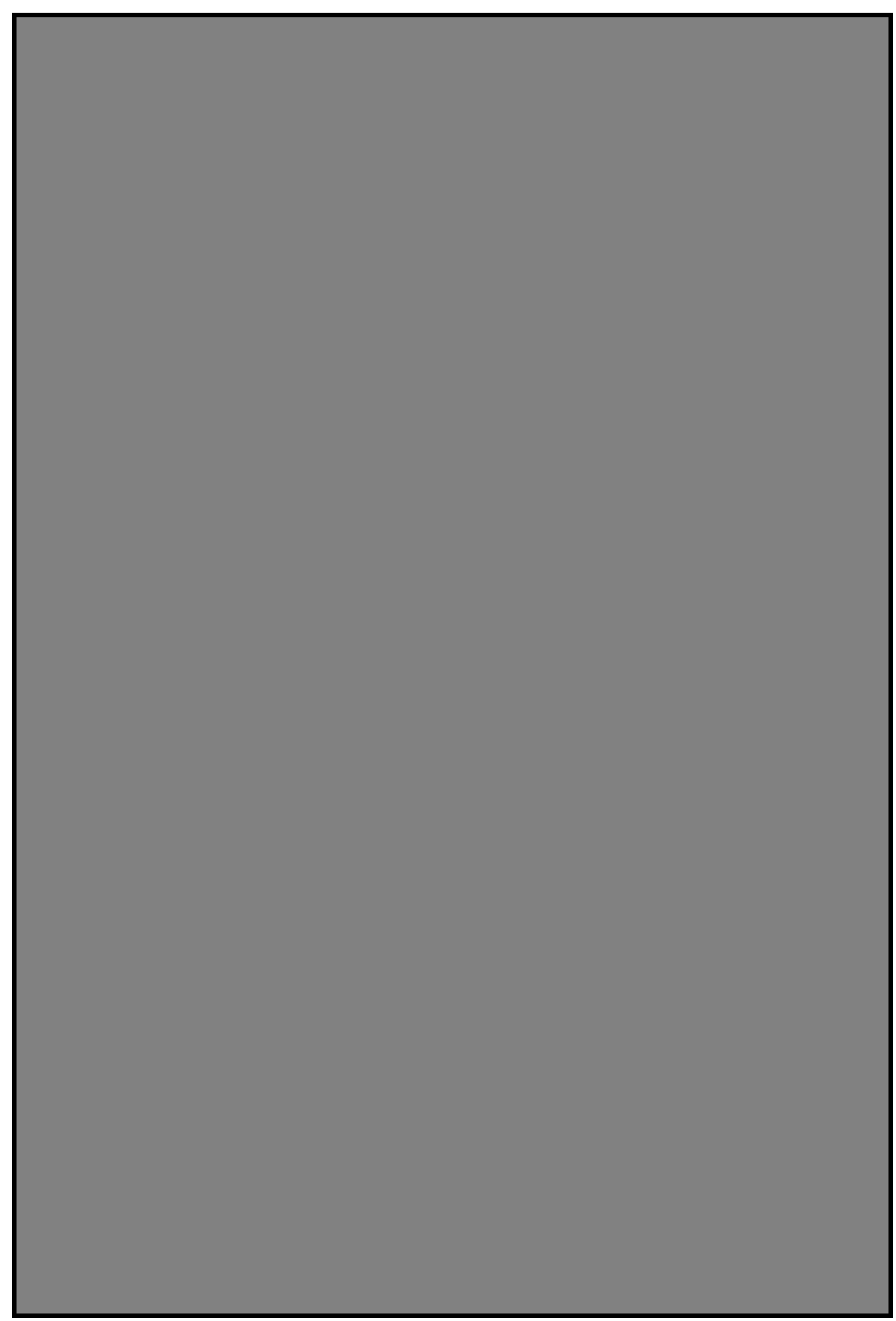

Abbildung 32: Dokumentationsbogen-Behandlung fazialer Dyskinesien.

Auf dem Behandlungsbogen wird bei jeder Injektion der Name des Patienten, die Diagnose und das Datum der Behandlung ein-getragen. Des Weiteren werden die Dosissymbole im Gesichts-schema zur Dokumentation der Topik der Injektionspunkte ein-gezeichnet. 


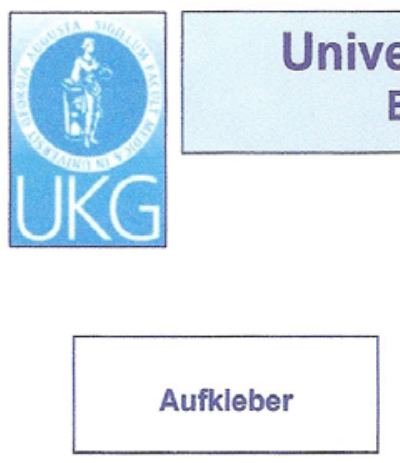

\section{Einverständniserklärung (Aufklärung)}

zur Botulinum-Toxin-Injektion bei Blepharospasmus, Spasmus facialis, spastisches Entropium, Fazialistic, Synkinesien:

Mit umseitiger Unterschrift bestätige ich, dass ich diese Erklärung gelesen und verstanden habe, dass ich die Möglichkeit hatte, mit dem Arzt deren Inhalt ausführlich und zu meiner Zufriedenheit zu besprechen, und dass ich mit dem beschriebenen Vorgehen einverstanden bin.

Die Unterschrift dieser Einverständniserklärung bedingt für mich keinerlei Verpflichtung, und ich darf ohne irgendwelche Verbindlichkeiten entscheiden, dass ich an dieser Behandlung nicht weiter teilnehmen werde oder eine andere Behandlungsform wähle. Dies gilt insbesondere für den Fall, dass es neue Informationen über Auswirkungen der Behandlung oder uber Komplikationen gibt. Ich wurde darüber aufgeklärt, dass ein augenchirurgisches bzw. neurochirurgisches Vorgehen eine Alternativmöglichkeit darstellt.

Wenn Nahrungsmittel, die einen Anteil von Botulinum-Toxin enthalten, gegessen werden, können sie eine Nahrungsmittelvergiftung (Botulismus) verursachen. Wird diese Substanz jedoch in der angewendeten extrem kleinen Menge in den Muskel gespritzt, verursacht sie eine vorübergehende Schwäche dieses Muskels, der eine zu starke Zugwirkung hat.

Herr Prof. Dr. med. R. Laskawi und/oder werden eine bestimmte Dosis von Botulinum-Toxin in einen oder mehrere Augenlid- bzw. Gesichtsmuskeln spritzen.

Ich werde mich bemühen, allen Anweisungen zu folgen und das Vorgehen zu erleichtern und ich werde unter ärztlicher Überwachung nach der Injektion bleiben, bis mir mitgeteilt wird, dass eine derartige sorgfältige Beobachtung nicht mehr notwendig ist.

Ich wurde darüber informiert, dass dieses Präparat auf dem Markt für die Indikation des Blepharospasmus, des hemifaszialen Spasmus und fokaler Dystonien zugelassen und für die anderen $0 . \mathrm{g}$. Indikationen nicht zugelassen ist (Off-label-use).

Ich wurde weiterhin daruber informiert, dass der Erfinder dieser Methode, Dr. Scott (Smith-KettlewellInstitute of Visual Science in San Francisco/USA) bzw. andere Mitarbeiter an diesem Forschungsvorhaben bislang mehrere tausend Patienten, die verschiedene Formen des Blepharospasmus hatten, mit Hilfe dieser Technik behandelt haben. Ich wurde weiterhin darüber informiert, dass diese Behandlung nicht das grundsătzliche Problem der unzweckmäßigen Versorgung des Muskels mit Nervenimpulsen löst, dass es sich hier aber um eine Methode handelt, den entstandenen Krampf der befallenen Muskeln zu lindern. Die Dauer dieses Effektes ist von Patient zu Patient unterschiedlich, hängt von der Dosierung ab und beträgt im Durchschnitt etwa 3 Monate.

Abbildung 33: Einverständniserklärung Botulinumtoxin-Therapie (Seite 1) 
Fortsetzung der Einverständniserklärung zur Botulinum-Toxin-Injektion bei Blepharospasmus, Spasmus facialis, spastischem Entropium, Fazialistic, Synkinesien:

Weil die 1. Injektion mit einer verhăltnismäßig geringen Dosis vorgenommen wird, hat sie typischerweise einen eher unzureichenden Effekt, und normalerweise sind zusătzliche Injektionen mit derselben Dosis oder mit höheren Dosen erforderlich.

Ich bin jedoch darüber aufgeklärt worden, dass der Effekt, der auch eine vorübergehende Schwäche von anderen Gesichtsmuskeln bewirken kann, größer sein kann als vorgesehen.

\section{Nebenwirkungen:}

Ich wurde uber die Risiken, die mit dieser Behandlung verbunden sind, aufgeklärt. Insbesondere können auftreten: vorubergehendes Herabhängen des/der Oberlider (Ptosis 11\%), Trockenheit der Augen, so dass Augentropfen notwendig werden (4,5\%), Tränen der Augen aufgrund der Schwäche des Oberlides (3,4\%), Schwierigkeit das Oberlid zu schließen (3,4\%), Lichtempfindlichkeit (1,4\%). Andere Nebenwirkungen werden sehr selten berichtet: Ektropium (Auswärtskehren des Unterlides), Hornhautentzündungen, Doppeltsehen, Blutungen, Schwellungen des Lides, Anstrengung beim Sehen und Entropium (Einwärtskehren des Lides). Diese letztgenannten Störungen treten sämtlich in weniger als $1 \%$ auf. Bei der Behandlung des Spasmus facialis kann gelegentlich eine Störung der Lippenbewegung auftreten, die Schwierigkeiten beim Sprechen zur Folge haben kann. Alle diese Medikamentenwirkungen sind vorübergehender Natur. Systemische Nebenwirkungen (Auswirkung auf den gesamten Körper außerhalb der behandelten Muskeln) sind nie aufgetreten. Wie bei Injektionen, die an anderen Stellen durchgeführt werden, können ein leichter Bluterguß und ein leichtes Unwohlsein gelegentlich auftreten. Mir wurde erklärt, dass diese Risiken ărztlich vertretbar sind.

Die Fragen nach generalisierten Muskelerkrankungen (z.B. Myasthenia gravis), derzeitiger Aminoglykosid-Antibiotika-Therapie (z.B. Streptomycin, Neomycin, Gentamicin) oder bestehender Schwangerschaft/Stillzeit habe ich verneint.

Ich wurde darauf hingewiesen, dass die grundsätzlich vorgeschriebene pharmakologisch-toxikologische Prüfung am Tier stattgefunden hat und sich dabei keine Bedenken gegen die Anwendung des Prüfpräparates am Menschen ergeben haben.

Aufgrund dieser Informationen erkläre ich mich damit einverstanden, dass die vorgenannte Behandlung einschließlich der dafür notwendigen ärztlichen Untersuchungen an mir durchgeführt wird, wobei ich mir vorbehalte, meine Einwilligung jederzeit widerrufen zu können. Ferner erkläre ich meine Bereitschaft, mich an die ärztlichen Anordnungen für die Zeit vor, während und nach der Behandlung zu halten.

Meine persönlichen Daten und meine Krankengeschichte unterliegen den üblichen Bestimmungen über die ärztliche Schweigepflicht. Ich bin damit einverstanden, dass Photographien, auf denen ich erkennbar bin sowie die Beschreibung meiner Krankengeschichte in medizinischen Zeitschriften, Büchern und anderen Publikationen oder Medien veröffentlicht werden oder auf wissenschaftlichen Fachtagungen vorgetragen werden.

Hiermit versichere ich die Übernahme entstandener Behandlungskosten im Falle einer Nichterstattung durch die Krankenkasse.

Göttingen, den

Abbildung 34: Einverständniserklärung Botulinumtoxin-Therapie (Seite 2) 


\subsection{Dosisentwicklung am Injektionspunkt}

Es wurde jeder einzelne Punkt pro Injektionsdatum und Patient betrachtet. Es wurde ein Mittelwert aus allen Patienten pro Punkt und Injektionsdatum gebildet. Somit konnte eine Veränderung der Dosis von der ersten bis zur vierzigsten Injektion beobachtet werden. Diese Auswertung wurde für jede Erkrankungsgruppe separat durchgeführt.

Aus der Abbildung der Gruppe der Patienten mit Blepharospasmus geht hervor, dass mit der steigenden Behandlungsanzahl eine diskrete Steigerung der Botulinumtoxin-Dosis pro Punkt einhergeht. Bei der ersten Injektion wurde im Durchschnitt mit 2,5 Einheiten begonnen. Die Ausnahme hier ist der Injektionspunkt Nummer 6, bei dem die Dosis von Anfang an sehr gering gewählt worden ist (2,3 E). Sie steigt zwar auch etwas an (auf 2,4 und 3,1 E bei der 40. Behandlung), bleibt jedoch insgesamt am geringsten dosiert. Man kann auch beobachten, dass zwischen der 20. und der 30. Injektion die Punkte 2, 3, 4 und 5 eine Dosissteigerung erfahren. Die Dosis steigt hier von 2,4-2,6 E bei der ersten Behandlung über 3,2-3,5 E zwischen der 30. und 40. Behandlung auf 4,0 E bei der 40. Injektion an. Die übrigen Punkte 1 und 7-10, bleiben zunächst konstant, um dann wieder gering anzusteigen. Im Durchschnitt wurde dort 2,5-3,1 E injiziert. Die Injektionspunkte 9 und 10 wurden nur bis zu der 20 und 30.. Behandlung injiziert (siehe Abbildung 35 und Tabelle 23). 


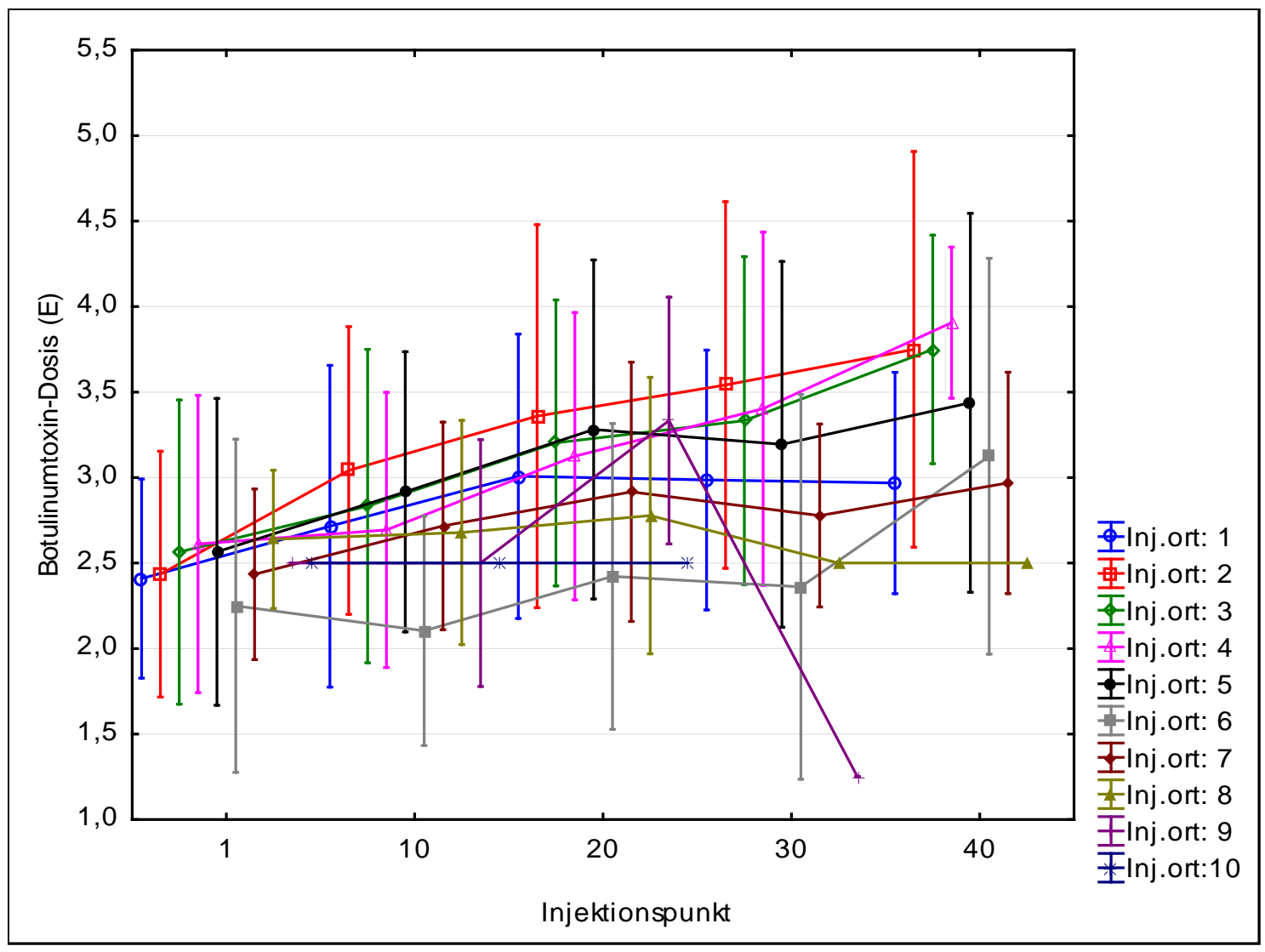

Abbildung 35: Mittlere Dosis mit Standardabweichung pro Injektionspunkt bei Patienten mit Blepharospasmus.

In dieser Abbildung erkennt man eine gewisse Tendenz zur Dosissteigerung. Die höchste Dosis wurde an den Punkten 1, 2, 3, 4, 5 und 7 mit 2,4 E beginnend bis zu 3,0-4,0 E bei der 40. Injektion appliziert. Die Punkte 8-10 blieben mit einem geringen Anstieg relativ konstant. Es wurde mit 2,5 bis 2,8 E im Durchschnitt behandelt. Die Dosis am Punkt 6 war am geringsten gewählt worden und beträgt im Durchschnitt 2,3 bis 3,1 E bei der letzten Injektion. Der Punkt 9 wurden nur bis zur 30. Behandlung injiziert, der Punkt 10 nur bis zur 20 Injektion. 


\begin{tabular}{|c|c|c|c|c|c|}
\hline \multicolumn{7}{|c|}{$\begin{array}{c}\text { Erkrankung: } \\
\text { Blepharospasmus }\end{array}$} \\
\hline \multirow{2}{*}{$\begin{array}{c}\text { Injektions- } \\
\text { punkt }\end{array}$} & \multicolumn{6}{|c|}{ Angabe der injizierten Dosis in E pro Behandlung } \\
\cline { 2 - 6 } & $\mathbf{1}$ & $\mathbf{1 0}$ & $\mathbf{2 0}$ & $\mathbf{3 0}$ & $\mathbf{4 0}$ \\
\hline $\mathbf{1}$ & 2,4 & 2,7 & 3 & 2,9 & 3,0 \\
\hline $\mathbf{2}$ & 2,4 & 3 & 3,3 & 3,5 & 4 \\
\hline $\mathbf{3}$ & 2,5 & 2,8 & 3,2 & 3,3 & 4 \\
\hline $\mathbf{4}$ & 2,6 & 2,7 & 3,1 & 3,4 & 4 \\
\hline $\mathbf{5}$ & 2,5 & 2,9 & 3,3 & 3,2 & 3,4 \\
\hline $\mathbf{6}$ & 2,3 & 2,1 & 2,4 & 2,3 & 3,1 \\
\hline $\mathbf{7}$ & 2,4 & 2,7 & 2,9 & 2,7 & 3 \\
\hline $\mathbf{8}$ & 2,6 & 2,7 & 2,8 & 2,5 & 2,5 \\
\hline $\mathbf{9}$ & 2,5 & 2,5 & 3,3 & 1,25 & \\
\hline $\mathbf{1 0}$ & 2,5 & 2,5 & 2,5 & & \\
\hline
\end{tabular}

Tabelle 23: Verlauf der mittleren Dosis mit pro Injektionspunkt beim Blepharospasmus.

Die Angaben der mittleren Dosis pro Injektionspunkt beziehen sich auf die erste, zehnte bis zu der vierzigsten Behandlung.

Eine Betrachtung dieser Art konnte auch bei Patienten mit einem Spasmus facialis gemacht werden. Hier wurden der periorbitale Bereich und der periorale Bereich mit dem Platysmabereich getrennt analysiert. Im Bereich des M. orbicularis oculi wurde im Mittel mit einer Dosis zwischen 1,8 und 2,5 Einheiten Botulinumtoxin begonnen. Die Punkte Nummer 1 und 6 wurden mit 1,5 bis 1,8 Einheiten injiziert. Auch hier ist die Dosis im Laufe der Behandlung auf 2,5 E angestiegen, blieb jedoch bis zu der 30. Injektion insgesamt am geringsten 1,8-2,2 E. Zwischen der 16. und 25. Behandlung kam es im Durchschnitt zu Dosisschwankungen zwischen 2,1-2,5 E, die jedoch dann bis zu der 30. Injektion auf einem bestimmten Niveau relativ konstant blieben. Nach der 30. Injektion kam es erneut zum vereinzelten Anstieg der Einzeldosis auf 3,1 und 3,7 E pro Punkt. Dies betraf fast ausschließlich die Punkte 5, 9 und 10. Die restlichen Injektionspunkte erhielten im Mittel wieder 2,5 Einheiten, also die Anfangsdosis (siehe Abbildung 36 und Tabelle 24). 
Im Platysmabereich wurde immer die gleich bleibende Dosis von 2,5 Einheiten verwendet, die sich über alle Behandlungsjahre konstant hielt. Die Injektionspunkte im Bereich des Musculus zygomaticus und im perioralen Bereich unterlagen deutlichen Schwankungen. Am häufigsten wurde jeweils ein Punkt im Mund- und Kinnbereich mit einer Dosis zwischen 1,9 und 2,5 Einheiten behandelt. Die übrigen Punkte wurden in Abhängigkeit von der Beschwerdesymptomatik und dem Nebenwirkungsprofil injiziert, so dass die Schwankungen damit zu erklären sind (siehe Abbildung 37 und Tabelle 24).

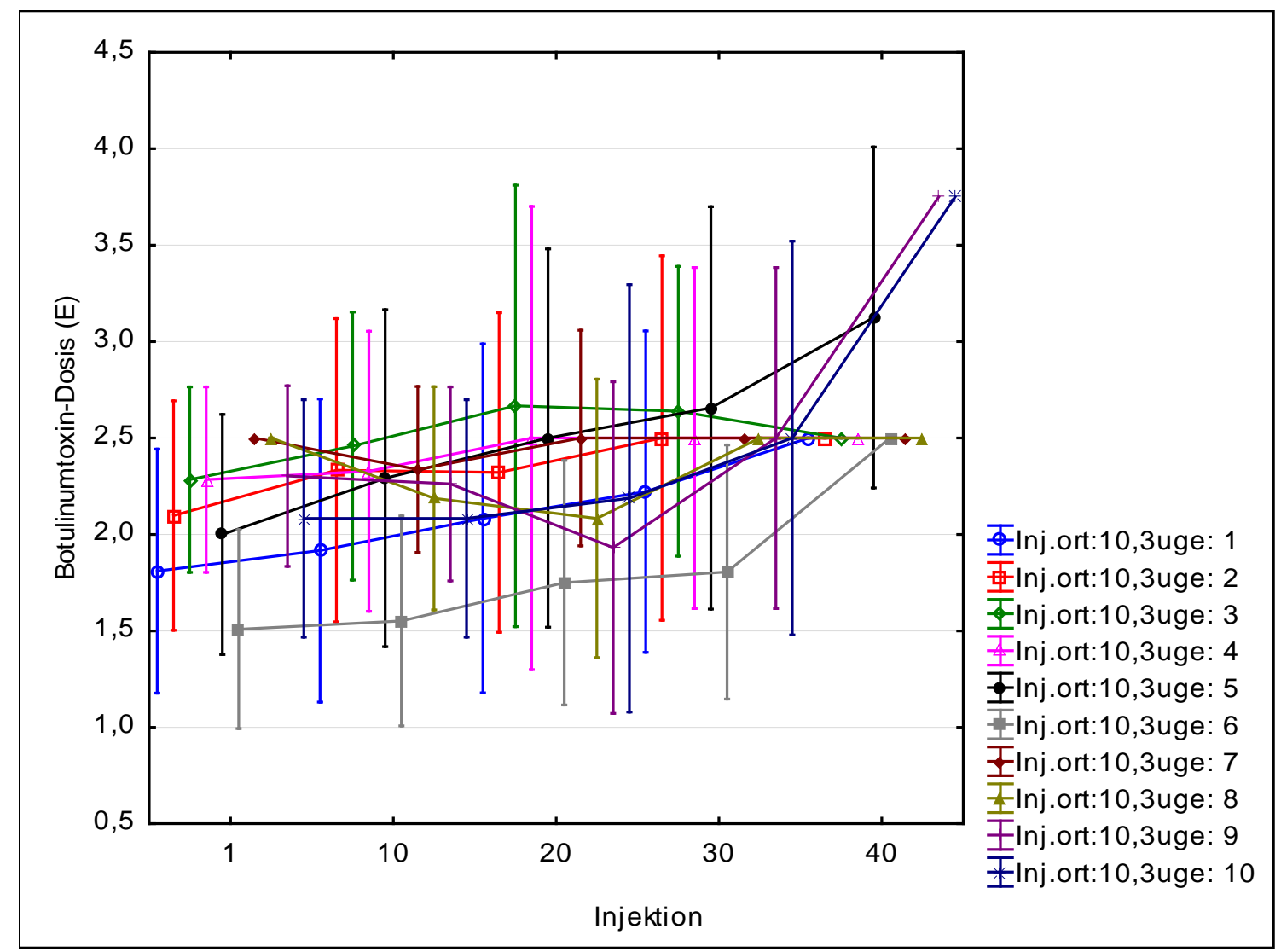

Abbildung 36: Mittlere Dosis mit Standardabweichung pro Injektionspunkt im periorbitalen Bereich bei Patienten mit Spasmus facialis.

An den Punkten Nummer 1 und 6 wurden mit der geringsten Dosis, 1,5 bis 1,8 E begonnen. Die Dosis ist im Laufe der Behandlung auf 2,5 E angestiegen, zeigt sich jedoch bis zu der 30. Injektion am geringsten mit 1,8-2,2 E. Die größten Dosisschwankungen befanden sich zwischen der 16. und 25. Behandlung mit 2,1-2,5 E, die jedoch dann bis zu der 30. Injektion auf einem bestimmten Niveau relativ 
konstant blieben. Ein erneuter Anstieg auf 3,75 E für Punkte 5,9 und 10 begann nach der 30. Injektion. Die übrigen Injektionspunkte erhielten die Anfangsdosis, also im Mittel 2,5 E.

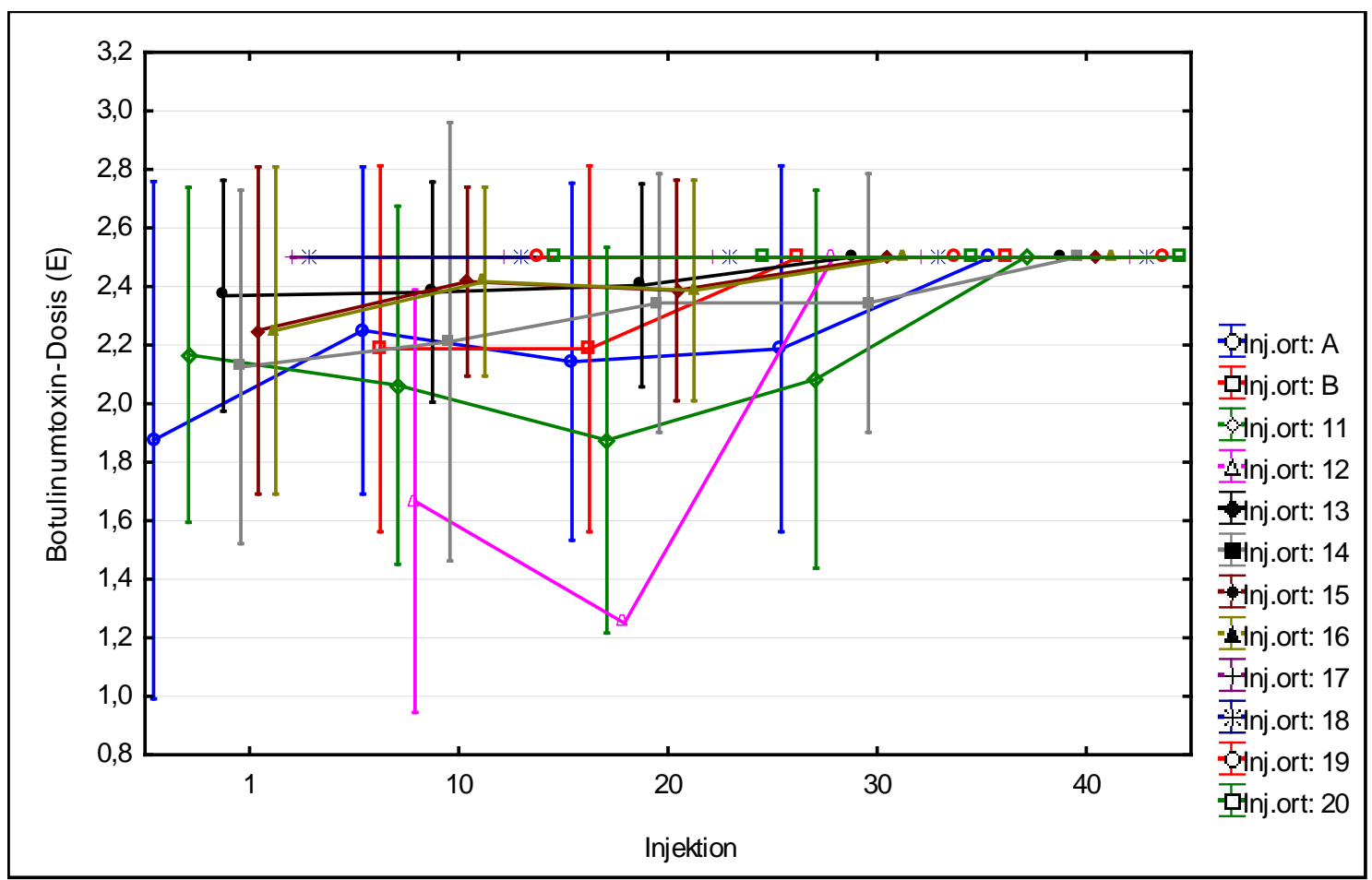

Abbildung 37: Mittlere Dosis mit Standardabweichung pro Injektionspunkt im perioralen und Platysmabereich bei Patienten mit Spasmus facialis.

Im Platysmabereich, Punkte 15-20, ist die Dosis über alle Behandlungsjahre mit 2,5 E injiziert worden. Der periorale Bereich und der M. zygomaticus sind abhängig von der Symptomatik behandelt worden, so dass sich hier die größten Schwankungen zeigen. Der häufigste Injektionsort war jeweils ein Punkt im Mundund Kinnbereich mit 2,3-2,5 E. 


\begin{tabular}{|c|c|c|c|c|c|}
\hline \multicolumn{6}{|c|}{$\begin{array}{c}\text { Erkrankung: } \\
\text { Spasmus facialis }\end{array}$} \\
\hline \multirow{2}{*}{$\begin{array}{l}\text { Injektions } \\
\text {-punkt }\end{array}$} & \multicolumn{5}{|c|}{ Angabe der injizierten Dosis in $\mathrm{E}$ pro Behandlung } \\
\hline & 1 & 10 & 20 & 30 & 40 \\
\hline 1 & 1,8 & 1,9 & 2,1 & 2,2 & 2,5 \\
\hline 2 & 2,1 & 2,3 & 2,3 & 2,5 & 2,5 \\
\hline 3 & 2,3 & 2,5 & 2,7 & 2,6 & 2,5 \\
\hline 4 & 2,3 & 2,3 & 2,5 & 2,5 & 2,5 \\
\hline 5 & 2 & 2,3 & 2,5 & 2,6 & 3,1 \\
\hline 6 & 1,5 & 1,5 & 1,7 & 1,8 & 2,5 \\
\hline 7 & 2,5 & 2,3 & 2,5 & 2,5 & 2,5 \\
\hline 8 & 2,5 & 2,2 & 2,1 & 2,5 & 2,5 \\
\hline 9 & 2,3 & 2,3 & 1,9 & 2,5 & 3,7 \\
\hline 10 & 2,1 & 2,0 & 2,2 & 2,5 & 3,7 \\
\hline A & 1,8 & 2,3 & 2,1 & 2,2 & 2,5 \\
\hline B & - & 2,2 & 2,2 & 2,5 & 2,5 \\
\hline 11 & 2,1 & 2 & 1,9 & 2,1 & 2,5 \\
\hline 12 & - & 1,7 & 1,2 & 2,5 & - \\
\hline 13 & 2,4 & 2,4 & 2,4 & 2,5 & 2,5 \\
\hline 14 & 2,4 & 2,2 & 2,3 & 2,3 & 2,5 \\
\hline $15-20$ & 2,5 & 2,4 & 2,4 & 2,5 & 2,5 \\
\hline
\end{tabular}

Tabelle 24: Verlauf der mittleren Dosis pro Injektionspunkt bei Spasmus facialis

Die Angaben der mittleren Dosis pro Injektionspunkt beziehen sich auf die erste, zehnte bis zu der vierzigsten Behandlung.

Die dritte Gruppe (Synkinesien-Gruppe) zeigt im Verlauf die meisten Abweichungen von der Durchschnittsdosis. Die erste Injektion im periorbitalen Bereich beginnt mit einer durchschnittlichen Dosis von 2,1 Einheiten. Diese fällt bis zu der zehnten Behandlung auf 1,2-1,7 Einheiten. Punkt 1 bleibt über die gesamten Jahre mit 1,6 E konstant. Die Punkte 6 und 8 zeigen im Allgemeinen über die Jahre eine Dosisminderung von 1,9 auf 1,2 E und 2,5- 1,2 E. Die übrigen Injektionsstellen unterliegen Schwankungen, die jedoch eine Tendenz zur 
Dosisminderung oder Konstanz zeigen. Der periorale Bereich und die Platysmaregion zeigen ähnlichen Dosisverlauf wie bei Patienten mit Spasmus facialis. Die Dosis am Platysma besteht im Mittel aus 2,5 Einheiten. Andere Punkte am M. zygomaticus und im perioralen Bereich zeigen dagegen deutliche Dosisschwankungen. Nach der 20. Behandlung ist im Diagramm zu sehen, dass die an die Beschwerdesymptomatik angepasste Dosis konstant geblieben ist (siehe Abbildung 38, 39 und Tabelle 25).

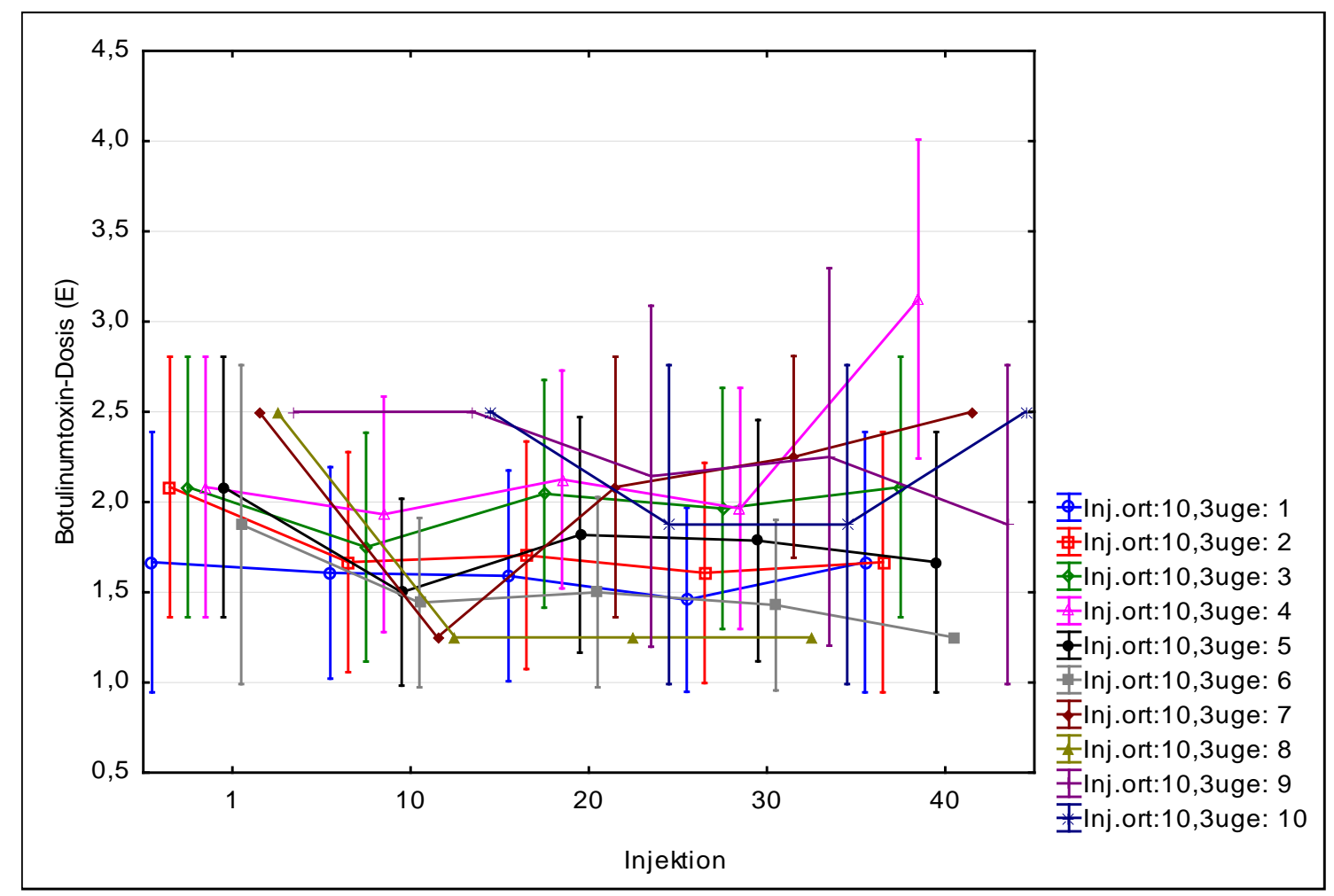

Abbildung 38: Mittlere Dosis mit Standardabweichung pro Injektionspunkt im periorbitalen Bereich bei Patienten mit Synkinesien

Die durchschnittliche Dosis in den ersten 10. Behandlungen fällt auf 1,2-1,7 E. Der Punkt 1 bleibt über die Jahre mit 1,6 E konstant. Bei den Punkten 6 und 8 ist über die Jahre eine Dosisminderung von 1,9 auf 1,2 E und 2,5- 1,2 E zu beobachten. Eine geringe Tendenz zur Dosisminderung oder Konstanz, jedoch mit Schwankungen, ist an den übrigen Injektionsstellen zu beobachten. 


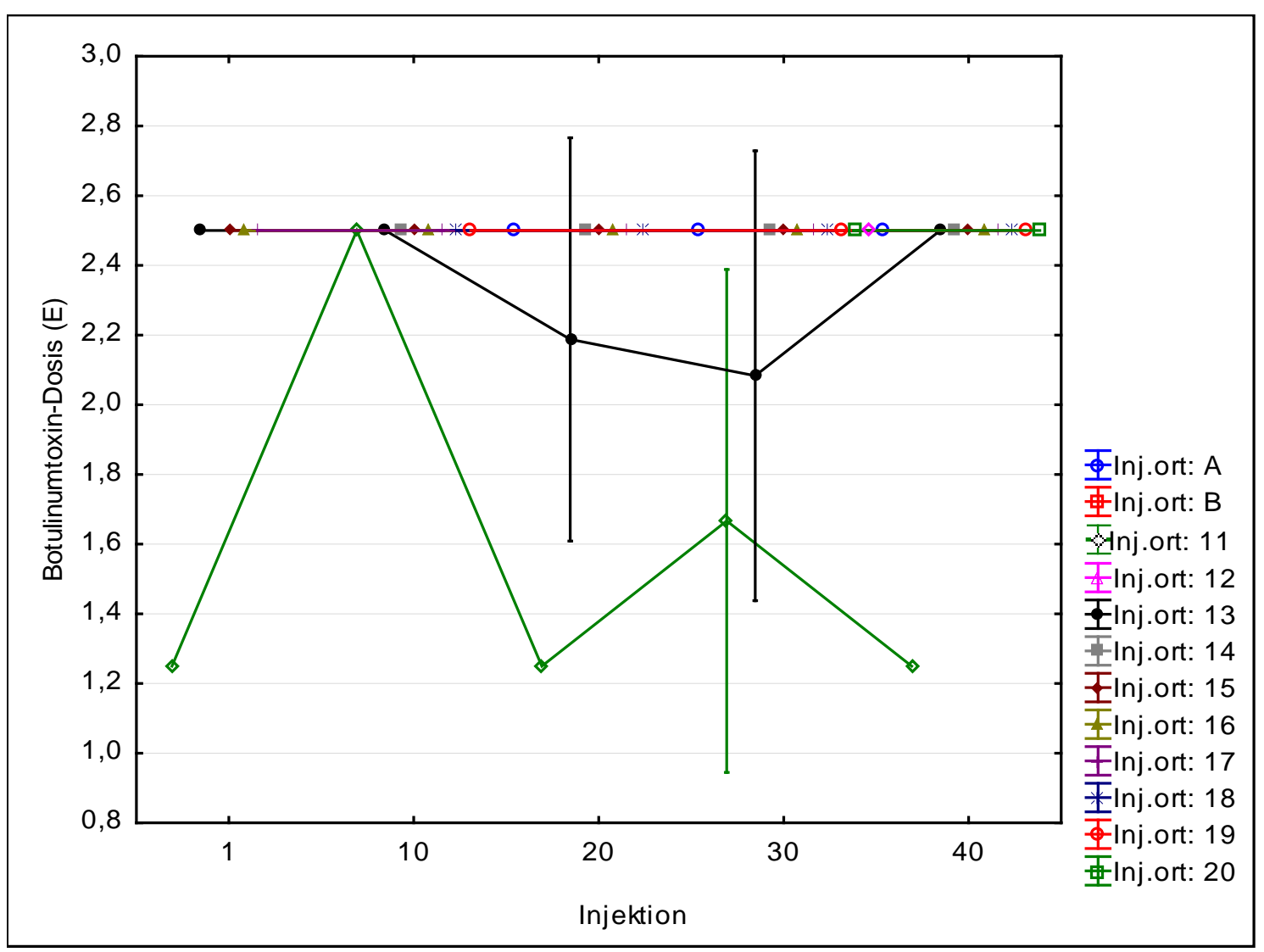

Abbildung 39: Mittlere Dosis mit Standardabweichung pro Injektionspunkt im perioralen und Platysmabereich bei Patienten mit Synkinesien

Die Dosis am Platysma besteht im Mittel aus 2,5 Einheiten. Die Punkte am Musculus zygomaticus und im perioralen Bereich zeigen Dosisveränderungen, vor allem nach der 10. Injektion. Nach der 20. Behandlung ist die mittlere Dosis konstant. 


\begin{tabular}{|c|c|c|c|c|c|}
\hline \multicolumn{6}{|c|}{$\begin{array}{l}\text { Erkrankung: } \\
\text { Synkinesien }\end{array}$} \\
\hline \multirow{2}{*}{$\begin{array}{c}\text { Injektions } \\
\text {-punkt }\end{array}$} & \multicolumn{5}{|c|}{ Angabe der injizierten Dosis in E pro Behandlung } \\
\hline & 1 & 10 & 20 & 30 & 40 \\
\hline 1 & 1,6 & 1,6 & 1,6 & 1,5 & 1,6 \\
\hline 2 & 2,1 & 1,7 & 1,7 & 1,6 & 1,6 \\
\hline 3 & 2,1 & 1,7 & 2 & 2 & 2,1 \\
\hline 4 & 2,1 & 2 & 2,1 & 2 & 3,1 \\
\hline 5 & 2,1 & 1,5 & 1,8 & 1,9 & 1,6 \\
\hline 6 & 1,9 & 1,4 & 1,5 & 1,4 & 1,2 \\
\hline 7 & 2,5 & 1,2 & 2,1 & 2,2 & 2,5 \\
\hline 8 & 2,5 & 1,2 & 1,2 & 1,25 & - \\
\hline 9 & 2,5 & 2,5 & 2,1 & 2,25 & 1,9 \\
\hline 10 & - & 2,5 & 1,9 & 1,9 & 2,5 \\
\hline A & - & - & 2,5 & 2,5 & 2,5 \\
\hline B & - & - & - & - & - \\
\hline 11 & 1,5 & 2,5 & 1,2 & 1,6 & 1,25 \\
\hline 12 & - & & & - & - \\
\hline 13 & 2,5 & 2,5 & 2,2 & 2 & 2,5 \\
\hline 14 & - & 2,5 & 2,5 & 2,5 & 2,5 \\
\hline $15-20$ & 2,5 & 2,5 & 2,5 & 2,5 & 2,5 \\
\hline
\end{tabular}

Tabelle 25: Verlauf der mittleren Dosis pro Injektionspunkt bei Synkinesien Die Angaben der mittleren Dosis pro Injektiospunkt beziehen sich auf die erste, zehnte bis zu der vierzigsten Behandlung.

Man muss bei dieser Darstellung jedoch bedenken, dass zu den unterschiedlichen Zeitpunkten auch sehr unterschiedliche und auch kleine Patientenanzahl vorhanden ist. Somit ist die Aussagekraft eingeschränkt. 


\section{Literaturverzeichnis}

Ainsworth JR, Kraft SP (1995): Long-term changes in duration of relief with botulinum toxin treatment of essential blepharospasm and hemifacial spasm. Ophthalmology 102, 2036-40

Aoki KR (2001): Pharmacology and immunology of botulinum toxin serotypes. J Neurol $\underline{248}$ Suppl 1:3-10

Arnon SS, Midura TF, Clay SA, Wood RM, Chin J (1977): Infant botulism. Epidemiological, clinical, and laboratory aspects. JAMA 237, 1946-1951

Arnon SS, Schechter R, Inglesby TV, Henderson DA, Barlett JG, Ascher MS, Eitzen E, Fine AD, Hauer J, Layton M, Lillibridge S, Osterholm MT, O`Toole T, Parker G, Perl TM, Russell PK, Swerdlow DL, Tonat K (2001): Botulinum toxin as a biological weapon. JAMA 285, 1059-1070

Baldwin M, Barbieri JT (2009): Association of botulinum neurotoxins with synaptic vesicle protein complexes. Toxicon $\underline{54}, 570-574$

Barker FG, Janneta PJ, Bissonette DJ, Shields PT, Larkins MV, Jho HD (1995): Microvascular

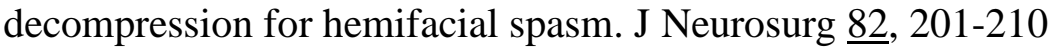

Beurskens CH, Heymans PG (2004): Physiotherapy in patients with facial nerve paresis: description of outcomes. Am J Otolaryngol 25, 394-400

Beurskens CH, Heymans PG, Oostendorp RA (2006): Stability of benefits of mime therapy in sequelae of facial nerve paresis during a 1-year period. Otol Neurotol 27, 1037-1042

Beurskens CH, Oosterhof J, Nijhuis-van der Sanden MW (2010): Frequency and location of synkineses in patients with peripheral facial nerve paresis. Otol Neurotol $\underline{31}$ (4), 671-675

Blasi J, Chapman ER, Link E, Binz T, Yamasaki S, De Camilli P, Südhof TC, Niemann H, Jahn R (1993): Botulinum neurotoxin A selectively clave the synaptic protein SNAP-25. Nature $\underline{365}, 160-163$ 
Boroojerdi B, Ferbert A, Schwarz M, Herath H, Noth J (1998): Botulinum toxin treatment of Synkinesia and hyperlacrimation after facial palasy. J Neurol Neurosurg Psychiatr $\underline{65}$, 111-114

Breit S, Heckmann M (2000): Botulinumtoxin. Ein Neurotoxin zur dermatologischen Therapie. Hautarzt 51, 874-891

Brin MF (2009): Basic and clinical aspects of Botox ${ }^{\circledR}$. Toxicon 54, 676-682

Brunger AT, Rummel A (2009): Receptor and substrate interactions of clostridial neurotoxins. Toxicon $\underline{54}$, 550-560

Calace P, Cortese G, Piscopo R, Della Volpe G, Gagliardi V, Magli A, De Berardinis T (2003): Treatment of blepharospasm with botulinum neurotoxin type A: long-term results. Eur J Ophthalmol 13, 331-336

Campbell E, und Keedy C (1947): Hemifacial spasm: a note on the etiology in two cases. Neurosurg $\underline{4(4)}$, 342-347

Campbell K, Collins MD, East AK (1993): Nucleotide sequence of the gene coding for Clostridium botulinum (Clostridium argentinense) type $G$ neurotoxin: genealogical comparison with other clostridial neurotoxins. Biochim Biophys Acta 1216, 487- 491

Cannon PS, Mackenzie KR, Cook AE, Leatherbarrow B (2010): Difference in response to botulinum toxin type A treatment between patients with benign essential blepharospasm and hemifacial spasm. Clin Experiment Ophthalmol 38, 688-691

Chen R-S, Lu C-S, Tsai C-H (1996): Botulinum toxin A injection in the treatment of hemifacial spasm. Acta Neurol Scand $\underline{94}, 207-211$

Cherington M (2004): Botulism: update and revive. Sem Neurol 24, 155-163

Chertow DS, Tan ET, Maslanka SE, Schulte J, Bresnitz EA, Weisman RS, Bernstein J, Marcus SM, Kumar S, Malecki J, Sobel J, Braden CR (2006): Botulism in 4 adults following cosmetic injections with an unlicensed, highly concentrated botulinum preparation. JAMA 296, 2476-2479 
Chua CN, Quhill F, Jones E, Voon LW, Ahad M, Rowson N (2004): Treatment of abberant facial nerve regeneration with botulinum toxin A. Orbit $\underline{23}, 213-218$

Coad JE, Wirtschafter JD, Haines SJ, Heros RC, Perrone T (1991): Familial hemifacial spasm associated with arterial compression of the facial nerve. J Neurosurg $\underline{74}$, 290-296

Coscarelli JM (2010): Essential blepharospasm. Semin Ophthalmol 25, 104-108

Defazio G, Abbruzzese G, Girlanda P, Vacca L, Currà A, De Salvia R, Marchese R, Raineri R, Roselli F, Livrea P, Berardelli A (2002): Botulinum toxin A treatment for primary hemifacial spasm: a 10-year multicenter study. Arch Neurol $\underline{59}$, 418-420

De Paiva A, Meunier FA, Molgó J, Aoki KR, Dolly JO (1999): Functional repair of motor endplates after botulinum neurotoxin type A poisoning: biphasic switch of synaptic activity between nerve sprouts and their parent terminals. Proc Natl Acad Sci U S A. 므, 3200-3205

Dressler D (2008): Botulinum toxin drugs: future developments. J Neural Transm $\underline{115}$, 575577

Dressler D, Benecke R (2003): Autonomic side effects of botulinum toxin type B treatment of cervical dystonia and hyperhidrosis. Eur Neurol $\underline{49}$, 34-38

Dressler D, Benecke R: Antikörpervermitteltes Versagen der Botulinumtoxin-Therapie. In: Botulinumtoxin- Therapie im Kopf- Hals- Bereich; 2., aktualisierte erweiterte Auflage; Hrsg. Rainer Laskawi, Peter Roggenkämper; Urban and Vogel München 2004, 58-70

Dressler D, Bigalke H (2004): Antibody-induced failure of botulinum toxin type B therapy in de novo patients. Eur Neurol 52, 132-135

Dressler D, Bigalke H: Pharmacology of Botulinum toxin drugs. In: Manual of Botulinum Toxin Therapy; Hrsg. Daniel Truong, Dirk Dressler, Mark Hallett, Cambridge University Press, New York 2009, 13-22

Dutton JJ, Buckley EG (1988): Long-term results and complications of botulinum A toxin in the treatment of blepharospasm. Ophthalmology $\underline{95}$, 1529-34 
Erbguth FJ (2008): From poison to remedy: the chequered history of botulinum toxin. J Neural Transm $\underline{115}, 559-565$

Fachinformation: Botox ${ }^{\circledR} 100$ Allergan-Einheiten. Pulver zur Herstellung einer Injektionslösung. Allergan Pharmaceuticals Ireland 2009

Fischer A, Montal M (2007): Crucial role of the disulfide bridge between botulinum neurotoxin light and heavy chains in protease translocation across membranes. J Biol Chem 282, 29604-29611

Foran PG, Davletov B, Meunier FA (2003): Getting muscles moving again after botulinum toxin: novel therapeutic challenges. Trends Mol Med $\underline{9}$, 291-298

Frei K, Roggenkämper P: Treatment of hemifacial spasm. In: Manual of Botulinum Toxin Therapy; Hrsg. Daniel Truong, Dirk Dressler, Mark Hallett; Cambridge University Press, New York 2009, 43-47

Frevert J (2009): Xeomin ${ }^{\circledR}$ : an innovative new botulinum toxin type A. Eur J Neurol 16, 1113

Friedman A, Jamrozik Z, Bojakowsky J (1989): Familial hemifacial spasm. Mov Disord $\underline{4}$, 213-218

Frueh BR, Musch DC (1986): Treatment of facial spasm with botulinum toxin. An interim report. Ophthalmology $\underline{93}$, 917-923

Galvez-Jimenez N, Hanson MR, Desai M (2001): Unusual causes of hemifacial spasm Sem Neurol 21, 75-83

Gill HS, Kraft SP (2010): Long-term efficacy of botulinum A toxin for blepharospasm and hemifacial spasm. Can J Neurol Sci $\underline{37}$, 631-636

Glaser DA: Cosmetic uses of botulinum toxin. In: Manual of Botulinum Toxin Therapy; Hrsg. Daniel Truong, Dirk Dressler, Mark Hallett; Cambridge University Press, New York, 2009, $133-142$ 
Grablowitz D: Plastisch-ästhetische Indikationen im Gesichtsbereich. In: BotulinumtoxinTherapie im Kopf-Hals-Bereich; 2., aktualisierte erweiterte Auflage; Hrsg. Rainer Laskawi, Peter Roggenkämper; Urban and Vogel, München 2004, 118

Green P, Fahn S, Diamond B (1994): Development of resistance to botulinum toxin type A in patients with torticollis. Mov Disord $\underline{9}$, 213-217

Grumelli C, Verderio C, Pozzi D, Rossetto O, Montecucco C, Matteoli M (2005): Internalisation and mechanism of clostridial toxins in neurons. NeuroToxicol 26, 761-767

Hsiung GY, Das SK, Ranawaya R, Lafontaine AL, Suchowersky O (2002): Long-term efficacy of botulinum toxin A in treatment of various movement disorders over a 10-year period. Mov Disord 17, 1288-1293

Huang W, Foster JA, Rogachefsky AS (2000): Pharmacology of Botulinum toxin. J Am Acad Dermatol $\underline{43}$, 249-259

Huh R, Han IB, Moon JY, Chang JW, Chung SS (2008): Microvascular decompression for hemifacial spasm: analyses of operative complications in 1582 consecutive patients. Surg Neurol $\underline{69}, 153-157$

Husseman J, Mehta RP (2008): Management of synkinesis. Facial Plast Surg 24, 242-249

Ito H, Ito H, Nakano S, Kusaka H (2007): Low-dose subcutaneous injection of botulinum toxin type A for facial synkinesis and hyperlacrimation. Acta Neurol Scand $\underline{115}$, 271-274

Jankovic J, Patel SC: (1983): Blepharospasm associated with brainstem lesions. Neurology $\underline{33}, 1237-1240$

Jankovic J, Schwartz K (1995): Response and immunoresistance to botulinum toxin injections. Neurology $\underline{45}, 1743-1746$

Jannetta PJ, Abassy M, Maroon JC, Ramos FM, Albin MS (1977): Etiology and definitive microsurgical treatment of hemifacial. J Neurosurg $\underline{47}$, 321-328

Jost WH, Kohl A (2001): Botulinum toxin: evidence-based medicine criteria in blepharospasm and hemifacial spasm. J Neurol 248 Suppl 1:21-24 
Kenney C, Jankovic J (2008): Botulinum toxin in the treatment of blepharospasm and hemifacial spasm. J Neural Transm 115, 585-591

Kollewe K, Mohammadi B, Dengler R, Dressler D (2010): Hemifacial spasm and reinnervation sykinesias: long-term treatment with Botox ${ }^{\circledR}$ or Dysport ${ }^{\circledR}$. J Neural Transm 117, $759-763$

Kreyden OP, Geiges ML, Böni R, Burg G (2000): Botulinumtoxin: Vom Gift zum Medikament. Ein historischer Rückblick. Hautarzt ㅁ1, 733-737

Kumaran D, Rawat R, Ludivico ML, Ahmed SA, Swaminathan S (2008): Structure - and substrate-based inhibitor design for Clostridium botulinum neurotoxin serotype A. J Biol Chem 283, 18883-18891

Laskawi R: Spasmus facialis. In: Botulinumtoxin- Therapie im Kopf-Hals-Bereich; 2., aktualisierte erweiterte Auflage; Hrsg. Rainer Laskawi, Peter Roggenkämper; Urban and Vogel, München 2004, 90-101

Laskawi R, Roggenkämper P: Botulinumtoxin-Therapie im Kopf-Hals-Bereich; 2., aktualisierte erweiterte Auflage; Hrsg. Rainer Laskawi, Peter Roggenkämper; Urban and Vogel, München 2004, 76

Laskawi R, Roggenkämper P: Synkinesien. In: Botulinumtoxin- Therapie im Kopf-HalsBereich; 2., aktualisierte erweiterte Auflage; Hrsg. Rainer Laskawi, Peter Roggenkämper; Urban and Vogel, München 2004, 102-113

Laskawi R, Damenz W, Roggenkämper P, Baetz A (1994): Botulinum toxin in patients with facial synkinesis. Eur Arch Otorhinolaryngol (Suppl), 195-199

Laskawi R, Demenz W, Roggenkämper P, Baetz A (1996): Botulinum toxin treatment in patients with facial synkinesis. Eur Arch Otorhinolaryngol (Suppl.), 195-199

Lovely TJ, Getch CC, Jannetta PJ (1998): Delayed facial weakness after microvaskular decompression of cranial nerve VII. Surg Neurol $\underline{50}$, 449-452 
Mauriello JA, Aljian J (1991): Natural history of treatment of facial dyskinesias with botulinum toxin: a study of 50 consecutive patients over seven years. Br J Ophthalmol $\underline{75}$, 737-739

Mauriello JA, Dhillon S, Leone T, Pakeman B, Mostafavi R, Yepez MC (1996a): Treatment selections of 239 patients with blepharospasm and Meige syndrom over 11 years. $\mathrm{Br} \mathrm{J}$ Ophthalmol 우, 1073-1076

Mauriello JA, Leone T, Dhillon S, Pakeman B, Mostafavi R, Yepez MC (1996b): Treatment choices of 119 patients with hemifacial spasm over 11 years. Clin Neurol Neurosurg $\underline{98,213-}$ 216

Mejia NI, Vuong KD, Jankovic J (2005): Long-term botulinum toxin efficacy, safety, and Immunogenicity. Mov Disord 20, 592-597

Montal M (2009): Translocation of botulinum neurotoxin light chain protease by the heavy chain protein-conducting channel. Toxicon $\underline{54}$, 565-569

Nagata S, Matsushima T, Fujii K, Fukui M, Curomatsu C (1992): Hemifacial spasm due to tumor, aneurysm, or arteriovenous malformation. Surg Neurol $\underline{38}$, 204-209

Neely G, Neufeld PS (1996): Defining functional limitation, disability, and societal limitations in patients with facial paresis: initial pilot questionnaire. Am J Otol 17, 340-342

Nielsen VK (1985): Electrophysiology of the facial nerve in hemifacial spasm: ectopic/ephaptic excitation. Muscle Nerve $\underline{8}$, 545-555

Nüßgens Z, Roggenkämper P (1995): Long-term treatment of blepharospasm with Botulinum toxin type A. German J Ophthalmol 또 363-367

Peckham EL, Lopez G, Shamim EA, Richardson SP, Sanku S, Malkani R, Stacy M, Mahant P, Crawley A, Singelton A, Hallett M (2011): Clinical features of patients with blepharospasm: a report of 240 patients. Eur J Neurol 18, 382-386

Pekmezovic T, Svetel M, Ivanovic N, Dragasevic N, Petrovic I, Tepavcevic DK, Kostic VS (2009): Quality of life in patients with focal dystonia. Clin Neurol Neurosurg $\underline{111}$, 161-164 
Pütz A: Empfehlungen zum Umgang mit der Substanz Botulinumtoxin. In: BotulinumtoxinTherapie im Kopf- Hals- Bereich; 2., aktualisierte erweiterte Auflage; Hrsg. Rainer Laskawi, Peter Roggenkämper; Urban and Vogel, München 2004, 46-54)

Roggenkämper P: Die Anwendung von Botulinum-Toxin bei essentiellem Blepharospasmus, Spasmus facialis, Fehlregeneration nach Fazialisparese und andere Indikationen. In: Huffmann G, Braune H-J, Henn K-H: Extrapyramidalmotorische Erkrankungen, EinhornPresse Verlag, Reinbek 1994, 422-427

Roggenkämper P (2007): Botulinumtoxin in der Augenheilkunde. Der Ophthalmologe 104, 758-758

Roggenkämper P, Laskawi R: Essentieller Blepharospasmus. In: Botulinumtoxin-Therapie im Kopf-Hals-Bereich; 2., aktualisierte erweiterte Auflage; Hrsg. Rainer Laskawi, Peter Roggenkämper; Urban and Vogel, München 2004, 73-89

Roggenkämper P, Laskawi R, Damenz W, Schröder M, Nüßgens Z (1990): Botulinum-ToxinBehandlung bei Synkinesien nach Fazialisparese. HNO 38, 295-297

Roggenkämper P, Laskawi R, Damenz W, Schröder M, Nüßgens Z (1991): Unwillkürlicher Lidschluß im Zuge einer Defektheilung nach Fazialisparese und dessen Behandlung mit Botulinus-Toxin. Klin Monatsbl Augenheilkd 4, 268-270

Roggenkämper P, Laskawi R, Damenz W, Schröder M, Nüßgens Z (1994): Orbicular synkinesis after facial paralysis: treatment with botulinum toxin. Documenta Ophthalmol $\underline{86}$, 395-402

Rohrbach S, Laskawi R (2004): Praktische Anwendung von Botulinumtoxin im Kopf- HalsBereich. HNO 모, 635-641

Sautter T, Herzog A, Hauri D, Schurch B (2001): Transient paralysis of the bladder due to wound botulism. Eur Urol 39, 610-612

Schiavo G, Matteoli M, Montecucco C. (2000): Neurotoxins affecting neuroexocytosis. Physiol Rev $\underline{80}$, 717-766 
Scott AB, MD, Kennedy RA, MD, Stubbs HA, (1985): Botulinum A toxin injection as a treatment for Blepharospasm. Arch Ophthalmol 103, 347- 150

Snir M, Weinberger D, Bourla D, Kristal-Shalit O, Dotan G, Axer-Siegel R (2003): Quantitative changes in botulinum toxin a treatment over time in patients with essential blepharospasm and idiopathic hemifacial spasm. Am J Ophthalmol 136, 99-105

Tan EK, Jankovic J (1999): Bilateral hemifacial spasm: a report of five cases and a literature review. Mov Disord 14, 345-349

Vitek JL (2002): Pathophysiology of dystonia: a neuronal model. Mov Disord 17, 49-62

Vogt T, Lüssi F, Paul A, Urban P (2008): Langzeittherapie fokaler Dystonien und des Hemispasmus facialis mit Botulinum-Toxin A. Nervenarzt 79, 912-917

Yoshimura DM , Michael J. Aminof, FRCP, Thomas A, Tami, Scot AB (1992): Treatment of hemifacial spasm with Botulinum toxin. Muscle Nerve 15, 1045-1049

Wang A, Jankovic J (1998): Hemifacial spasm: Clinical Findings and treatment. Muscle Nerve 21, 1740-1747 


\section{Danksagung}

Mein besonderer Dank gilt an dieser Stelle Prof. Dr. Rainer Laskawi für die Vergabe des Themas und seine stetige wissenschaftliche und moralische Unterstützung bei der Erstellung der vorgelegten Arbeit. Seine freundliche Einstellung und seine fast unbegrenzte Geduld waren mir immer eine sehr große Hilfe.

Die vorgelegte Arbeit wurde in der Abteilung Hals-Nasen-Ohrenheilkunde der Medizinischen Fakultät der Georg-August-Universität Göttingen durchgeführt. Daher gilt mein Dank ebenso dem Leiter der Abteilung Prof. Dr. C. Matthias und seinen Mitarbeitern für die Möglichkeit der Durchführung meiner Dissertation.

Ich möchte mich nicht zuletzt bei Herrn Simon Schneider vom Institut für Medizinische Statistik der Universität Göttingen für seine große Unterstützung bei der Durchführung der statistischen Berechnungen und Bearbeitung meiner Daten bedanken.

Nicht zuletzt möchte ich mich besonders bei den Personen bedanken, die ich während meiner Arbeit und Datenerhebung im Rahmen der „Spezialsprechstunde Botulinumtoxin“ kennen gelernt habe. Sämtliche Mitarbeiter dort waren mir gegenüber stets sehr freundlich und hilfsbereit. 


\section{Lebenslauf}

Ich wurde am 12.03.1977 als drittes Kind von Bogumil Niemczewski und seiner Ehefrau Stanislawa Niemczewska, geb. Chmiel, in Kwidzyn (Polen) geboren. Von 1984-1992 besuchte ich die Grundschule „Wladyslaw Gebik“ und anschließend von 1992-1996 das Allgemeine Gymnasium Nr. 1 in Kwidzyn. Hier legte ich 1996 das Abitur ab.

Von 1997-1998 arbeitete ich als Au- Pair bei einer Familie in Oldenburg, wo ich die Möglichkeit zur deutschen Sprachausbildung am Goethe- Institut bekam und sämtliche Prüfungen ablegte.

1998 begann ich im Wintersemester mein Medizinstudium an der Georg- August- Universität in Göttingen, wo ich im Jahr 2004 erfolgreich mein 2. Staatsexamen absolvieren konnte. Im Anschluss verbrachte ich mein praktisches Jahr zu zwei Dritteln am Evangelischen Krankenhaus Göttingen-Weende. Mein Wahlfach Augenheilkunde absolvierte ich in der Universitätsaugenklinik der Universität Göttingen. Mein 3. Staatsexamen legte ich am 02.11.2005 erfolgreich ab.

Im selben Jahr begann ich meine Doktorarbeit in der Abteilung Hals-Nasen-Ohrenheilkunde der Universität Göttingen unter meinem Doktorvater Prof. Dr. med. R. Laskawi. Von Januar bis März 2006 arbeitete ich als ärztliche Mitarbeiterin in der Botulinumtoxin-Sprechstunde der HNO-Poliklinik der Uniklinik Göttingen. Dabei hatte ich optimale Möglichkeiten, die Injektionstechniken bei den fazialen Dyskinesien zu erlernen.

Am 01.04.2006 begann ich meine Facharztausbildung in der Abteilung Augenheilkunde der Georg-August-Universität Göttingen. Die Ausbildung wurde im Herbst 2008 durch die Geburt meines ersten Sohnes und dem anschließenden Mutterschaftsurlaub unterbrochen. Vor der Geburt habe ich geheiratet und führe seitdem meinen doppelten Nachnamen NiemczewskaDreher. Seit Januar 2010 führe ich meine Ausbildung nun wieder fort. 